\title{
STABLE DECOMPOSITIONS OF $h p$-BEM SPACES AND AN OPTIMAL SCHWARZ PRECONDITIONER FOR THE HYPERSINGULAR INTEGRAL OPERATOR IN 3D
}

\author{
Michael Karkulik ${ }^{1}$, Jens Markus Melenk ${ }^{2}$ And Alexander Rieder ${ }^{3}$
}

\begin{abstract}
We consider fractional Sobolev spaces $H^{\theta}(\Gamma), \theta \in[0,1]$, on a $2 \mathrm{D}$ surface $\Gamma$. We show that functions in $H^{\theta}(\Gamma)$ can be decomposed into contributions with local support in a stable way. Stability of the decomposition is inherited by piecewise polynomial subspaces. Applications include the analysis of additive Schwarz preconditioners for discretizations of the hypersingular integral operator by the $p$-version of the boundary element method with condition number bounds that are uniform in the polynomial degree $p$.
\end{abstract}

1991 Mathematics Subject Classification. 65F08, 65N38, 41A35.

September 4, 2019.

\section{INTRODUCTION}

Fractional Sobolev spaces arise frequently in both analysis and numerical analysis when dealing with (fractional) partial differential or integral equations. We mention, for example, the classical boundary integral operators associated with the Laplacian that lead to the Sobolev space spaces $H^{1 / 2}(\Gamma)$. Similarly, for example in screen problems or fractional diffusion, the natural spaces are often given by Sobolev spaces encoding homogeneous Dirichlet conditions denoted by $\widetilde{H}^{\theta}(\Gamma)$.

When discretizing problems posed in such spaces, a standard ansatz space consists of globally continuous piecewise polynomials of degree $p$ on a mesh $\mathcal{T}$ that partitions $\Gamma$. An important tool in the numerical analysis of such a setting are stable decompositions of such discrete functions into local contributions.

In this work, we consider $2 \mathrm{D}$ surfaces $\Gamma \subset \mathbb{R}^{3}$ and propose a stable decomposition procedure of functions $u \in \widetilde{H}^{\theta}(\Gamma)$ into a global low-order contribution of piecewise linears/bilinears and functions with local support (see Theorem 2.5). This decomposition is constructed such that, if $u$ is a piecewise polynomial on a mesh $\mathcal{T}$, then the local contributions are also piecewise polynomials with the same degree distributions. Our construction accommodates variable polynomial degree distribution (i.e., the polynomial degree is allowed to vary from element to element) and "mixed" meshes consisting of triangles and quadrilaterals (see Theorem 2.6). Similar decompositions that emphasize the $p$-dependence have already appeared in the context of meshes consisting of quadrilaterals only in Pav94] for the case $\theta=1$ and [Heu99] for $\theta \in(0,1)$. For meshes consisting of triangles/tetrahedra only, the decomposition in the finite element case of $\theta=1$ was covered in SMPZ08 and recently a new decomposition was proposed in FW15 for general simplicial meshes. The decomposition of [FW15] is similar to our result in that it is also simultaneously stable in $L^{2}$ and $H^{1}$, which

Keywords and phrases: preconditioning high order BEM, stable localization, domain decomposition

${ }^{1}$ Departamento de Matemática, Universidad Técnica Federico Santa María, Valparaíso, Chile

${ }^{2}$ Institut für Analysis und Scientific Computing, Technische Universität Wien, Austria

${ }^{3}$ Institut für Analysis und Scientific Computing, Technische Universität Wien, Austria 
implies stability for general $\theta \in(0,1)$, although this simultaneous stability is not emphasized in [FW15] and its ramifications not explored.

An application of our stable decomposition is given by the analysis in Section 3.2 of an additive Schwarz preconditioner for the $p$-BEM applied to the hypersingular integral equation. The resulting condition number is shown to be uniformly bounded in the polynomial degree $p$ employed; here, mixed meshes consisting of triangles and quadrilaterals are allowed as well as a variable polynomial degree distribution. The numerical performance of this preconditioner is studied in FMPR15.

A second application of our decomposition result is given in Theorem 3.3, which identifies the interpolation space between spaces of piecewise polynomials (of arbitrary degree) equipped with weighted $L^{2}$-norms. In fact, in a subsequent work KMR we will use our decomposition to show that the interpolation space obtained by interpolating (using the $K$-method) between a space of piecewise polynomials equipped with the $L^{2}$ - and $H^{1}$-norm coincides with the same space equipped with the appropriate Sobolev norm, i.e.,

$$
\begin{aligned}
\left(\left(\widetilde{\mathcal{S}}^{\mathbf{p}, 1}(\mathcal{T}),\|\cdot\|_{L^{2}(\Gamma)}\right),\left(\widetilde{\mathcal{S}}^{\mathbf{p}, 1}(\mathcal{T}),\|\cdot\|_{\widetilde{H}^{1}(\Gamma)}\right)\right)_{\theta, 2} & =\left(\widetilde{\mathcal{S}}^{\mathbf{p}, 1}(\mathcal{T}),\|\cdot\|_{\widetilde{H}^{\theta}(\Gamma)}\right) \\
\left(\left(\mathcal{S}^{\mathbf{p}, 1}(\mathcal{T}),\|\cdot\|_{L^{2}(\Gamma)}\right),\left(\mathcal{S}^{\mathbf{p}, 1}(\mathcal{T}),\|\cdot\|_{H^{1}(\Gamma)}\right)\right)_{\theta, 2} & =\left(\mathcal{S}^{\mathbf{p}, 1}(\mathcal{T}),\|\cdot\|_{H^{\theta}(\Gamma)}\right) \quad \text { (equivalent norms), }
\end{aligned}
$$

here, the implied norm equivalences are independent of the polynomial degree distribution. This result of [KMR] will generalize the analysis of the single-element case in Mad89, BDM92, BDM10, BB94, and [BDM07, BDM10, Thm. 4.2] to general shape-regular meshes.

\section{Outline of the paper}

In view of the technical nature of the paper, we have collected the main results in Section 2, Applications such as the preconditioning of the $p$-version discretization of the hypersingular integral operator are given in Section 3. The remainder of the paper is devoted to the proof of the main result, namely, the decomposition of a function $u$ into local components. The decomposition is such that the local components are supported on the patches of a mesh, i.e., the union of elements meeting at a vertex or an edge. It is performed in several steps. In a first step, a piecewise linear contribution $u_{1}$ is determined using a Scott-Zhang interpolant. The primary purpose of this contribution is to remove the $h$-dependence of the decomposition, i.e., the difference $u-u_{1}$ can be localized with control of the constants uniformly in the size of the mesh patches. In a second step, "vertex contributions" for each vertex $V$ of the mesh are determined with the aid of averaging operators. The vertex contributions have two important properties: a) the support of each vertex contribution is the corresponding vertex patch $\omega_{V}$ and b) the function value at $V$ is reproduced if the function is continuous at $V$. We collect these vertex contributions in the function $u_{2}$. Then the function $u-u_{1}-u_{2}$ vanishes in the vertices of the mesh. A second averaging operator is applied to this function for each edge to create "edge contributions" supported by the patches $\omega_{e}$ associated with the edges. These are collected in a function $u_{3}$. The difference $u-u_{1}-u_{2}-u_{3}$ is then a function vanishing on the skeleton of the mesh and therefore consists of local "element contributions".

The stability properties of the averaging operators aplied in these steps of the decomposition have to be analyzed. In preparation to that analysis, we address in Section 4 the issue that full Sobolev norms do not scale conveniently under (affine) changes of variables but that seminorms do. Since we define fractional Sobolev spaces by interpolation, we present and analyze interpolation spaces that are based on interpolating seminorms. These results come in two flavors: Section 4.1 focuses on the interpolation between Sobolev spaces, and Section 4.2 studies the case of interpolation between weighted Sobolev spaces with weight given by the distance from a point. The ensuing Section 5 develops averaging operators on the reference element $\widehat{K}$ with the following key property: "Vertex averaging operators" reproduce the function value in one corner of $\widehat{K}$ and "edge averaging operators" reproduce function values on one edge of $\widehat{K}$. The actual construction is performed in several steps since these operators should have the additional property that the "vertex averaging operator" should vanish on the edge opposite the vertex and the "edge averaging operator" should vanish on the other edges of $\widehat{K}$. Section $[6$ uses the averaging operators on the reference element $\widehat{K}$ to generate the vertex or edge contributions mentioned above. The general procedure for meshes consisting of triangles only is as follows: one selects from the (vertex or edge) patch a certain element $K^{\star}$. In the discrete 
case of piecewise polynomials, $K^{\star}$ is normally chosen to have the lowest polynomial degree present in the patch. (In some cases the element with a slightly higher polynomial degree is favored if it allows one to do the averaging on a triangle instead of a square). The corresponding vertex or edge contribution is defined for that element $K^{\star}$ by applying the averaging operator to the pullback to the reference element. For the remaining elements of the patch, the contribution is defined by copying the values from $K^{\star}$.

An important feature of the averaging operators for triangles is that polynomials of degree $p$ are mapped to polynomials of the same degree. In this way, the above decomposition procedure also yields a stable decomposition for spaces of piecewise polynomials. The presence of quadrilaterals in a mesh introduces significant complications. We define the averaging operators on the reference square in terms of the averaging operators on a triangular part of the square combined with mapping this triangular part to the full square using a Duffy transformation. When applied to polynomials of degree $p$, this increases the polynomial degree to $2 p$. In the discrete case, we therefore introduce an additional Gauss-Lobatto interpolation step to get back to polynomials of degree $p$. Due to the lack of stability of the Gauss-Lobatto interpolation in $L^{2}$, the stability of the decomposition in fractional Sobolev norms $\widetilde{H}^{\theta}(\Gamma)$ cannot easily be inferred from stability in $L^{2}(\Gamma)$ and $\widetilde{H}^{1}(\Gamma)$ and an interpolation argument. Rather, a careful analysis in the local $\widetilde{H}^{\theta}$-spaces is necessary, which is done in the present paper.

\section{Notation, Assumptions, And main Results}

We will introduce the necessary notation and present the main results of the present work. Due to the technical nature of the results, all proofs are relegated to Section 6 .

$B_{r}(x)$ denotes the Euclidean ball with radius $r$ centered at $x$. The shorthand $a \lesssim b$ expresses $a \leq C b$ for a constant $C>0$ that does not depend on parameters of interest (in particular the mesh size $h$ and the polynomial degree $p$ ). The notation $a \sim b$ is short for $a \lesssim b$ in conjunction with $b \lesssim a$.

\subsection{Geometric and functional setting}

Let $\Omega \subseteq \mathbb{R}^{3}$ be a bounded Lipschitz domain and let $\Gamma \subseteq \partial \Omega$ be an open, connected subset of $\partial \Omega$ that stems from a Lipschitz dissection as described in [McL00, p. 99]. The Sobolev spaces $L^{2}(\partial \Omega)$ and $H^{1}(\partial \Omega)$ are defined as in [McL00, pp. 99] by use of Bessel potentials on $\mathbb{R}^{2}$ and liftings via the bi-Lipschitz maps that describe $\partial \Omega$. For any relatively open $\omega \subseteq \partial \Omega$, we define the space $L^{2}(\omega)$ of square integrable functions in the standard way. The spaces $H^{1}(\omega)$ and $\widetilde{H}^{1}(\omega)$ are defined by

$$
H^{1}(\omega):=\left\{\left.v\right|_{\omega}: v \in H^{1}(\partial \Omega)\right\}, \quad \widetilde{H}^{1}(\omega):=\left\{v: E_{0, \omega} v \in H^{1}(\partial \Omega)\right\},
$$

where $E_{0, \omega}$ denotes the operator that extends a function defined on $\omega$ to a function on $\partial \Omega$ by zero. We recall that for each $u \in H^{1}(\partial \Omega)$ we can define the surface gradient $\nabla u \in L^{2}(\partial \Omega)$. It can be checked that for (relatively) open $\omega \subseteq \partial \Omega$ and $u \in H^{1}(\omega)$ or $u \in \widetilde{H}^{1}(\omega)$ the surface gradient $\nabla u$ is also well-defined on $\omega$ and depends only on the function values of $u$ on $\omega$. With the surface gradient in hand, we introduce the seminorm and norm

$$
|u|_{H^{1}(\omega)}:=\|\nabla u\|_{L^{2}(\omega)}, \quad\|u\|_{H_{h}^{1}(\omega)}^{2}:=\frac{1}{\operatorname{diam}(\omega)^{2}}\|u\|_{L^{2}(\omega)}^{2}+|u|_{H^{1}(\omega)}^{2} .
$$

Fractional Sobolev spaces are defined by interpolation via the $K$-method. For two Banach spaces $\left(X_{0},\|\cdot\|_{0}\right)$, $\left(X_{1},\|\cdot\|_{1}\right)$ with continuous embedding $X_{1} \subseteq X_{0}$ and fixed $\theta \in(0,1)$, define the $K$-functional by $K^{2}(t, u):=$ $\inf _{v \in X_{1}}\|u-v\|_{0}^{2}+t^{2}\|v\|_{1}^{2}$ and the interpolation norm by

$$
\|u\|_{\left(X_{0}, X_{1}\right)_{\theta, 2}}^{2}:=\int_{t=0}^{\infty} t^{-2 \theta} K^{2}(t, u) \frac{d t}{t}=\int_{t=0}^{\infty} t^{-2 \theta}\left(\inf _{v \in X_{1}}\|u-v\|_{0}^{2}+t^{2}\|v\|_{1}^{2}\right) \frac{d t}{t}
$$

together with the convention that for $\theta=0$ or $\theta=1$ we set $\|u\|_{\left(X_{0}, X_{1}\right)_{\theta, 2}}^{2}=\|u\|_{X_{\theta}}^{2}$.

We then define the interpolation space $\left(X_{0}, X_{1}\right)_{\theta, 2}:=\left\{u \in X_{0}:\|u\|_{\left(X_{0}, X_{1}\right)_{\theta, 2}}<\infty\right\}$. In this way, the spaces $H^{\theta}(\omega):=\left(L^{2}(\omega), H^{1}(\omega)\right)_{\theta, 2}, \widetilde{H}^{\theta}(\omega):=\left(L^{2}(\omega), \widetilde{H}^{1}(\omega)\right)_{\theta, 2}$ as well as $H_{h}^{\theta}(\omega):=\left(L^{2}(\omega), H_{h}^{1}(\omega)\right)_{\theta, 2}$, 
$\widetilde{H}_{h}^{\theta}(\omega):=\left(L^{2}(\omega), \widetilde{H}_{h}^{1}(\omega)\right)_{\theta, 2}$ and their corresponding norms are defined. Occasionally, it will be more convenient to work with seminorms instead of full norms. For two Banach spaces $X_{0}, X_{1}$ with $\|\cdot\|_{X_{1}}^{2}=$ $|\cdot|_{X_{1}}^{2}+c^{2}\|\cdot\|_{X_{0}}^{2}$, we define a seminorm by

$$
|u|_{\left(X_{0}, X_{1}\right)_{\theta, 2}}^{2}=\int_{t=0}^{\infty} t^{-2 \theta}\left(\inf _{v \in X_{1}}\|u-v\|_{0}^{2}+t^{2}|v|_{1}^{2}\right) \frac{d t}{t} .
$$

For example, on the space $\widetilde{H}_{h}^{\theta}(\omega)$ we define in this way the seminorm

$$
|u|_{\widetilde{H}_{h}^{\theta}(\omega)}^{2}:=\int_{t=0}^{\infty} t^{-2 \theta}\left(\inf _{v \in \widetilde{H}^{1}(\omega)}\|u-v\|_{L^{2}(\omega)}^{2}+t^{2}|v|_{H^{1}(\omega)}^{2}\right) \frac{d t}{t}=|u|_{\widetilde{H}^{\theta}(\omega)}^{2} .
$$

We refer to Section 4.1 on how these seminorms relate to the full interpolation norms.

\subsection{Meshes and polynomial spaces}

In this section, we further restrict our assumptions on the surface $\Gamma$ and introduce the discretization into boundary elements which are the image of planar reference elements under suitable transformations.

We require that $\Gamma$ admits a suitable triangulation $\mathcal{T}$ into open subsets $K_{1}, \ldots, K_{|\mathcal{T}|}$, satisfying Assumption 2.1 below. As it is standard in FEM and BEM, each element $K_{i}$ is the image of some fixed reference element under an element map $F_{K_{i}}$. To that end, we define the reference triangle and square by

$$
\begin{aligned}
& \widehat{T}:=\{(\xi, \eta): 0<\xi<1, \quad 0<\eta<\xi\}, \\
& \widehat{S}:=\{(\xi, \eta): 0<\xi<1, \quad 0<\eta<1\} .
\end{aligned}
$$

Often, we will work with functions that are only defined on a subdomain $\omega \subseteq \Gamma$. Correspondingly, we write $\left.\mathcal{T}\right|_{\omega}:=\{K \in \mathcal{T}: K \subset \omega\}$ for the subtriangulation. Throughout the article, the triangulations and the element maps are required to satisfy the following assumption, which generalizes the usual concept of shape-regularity to elements on surfaces, where the element maps are mappings $\mathbb{R}^{2} \rightarrow \mathbb{R}^{3}$ :

Assumption 2.1. (1) For each element $K \in \mathcal{T}$, there exists $\widehat{K} \in\{\widehat{T}, \widehat{S}\}$ and an element map $F_{K}$ : $\widehat{K} \rightarrow K$ that is $C^{1}$ up to the boundary $\partial \widehat{K}$.

(2) The element maps $F_{K}$ are bijections $\bar{K} \rightarrow \bar{K}$.

(3) With $h_{K}:=\operatorname{diam} K$, the Gramian $G(x):=\left(F_{K}^{\prime}(x)\right)^{\top} \cdot F_{K}^{\prime}(x)$ has two eigenvalues $\lambda_{\min }(x), \lambda_{\max }(x)$. The shape regularity of $\mathcal{T}$ is characterized by the constant $\gamma$ that satisfies, for all $K \in \mathcal{T}$,

$$
\sup _{x \in \widehat{K}} \frac{h_{K}^{2}}{\lambda_{\min }(x)}+\frac{\lambda_{\max }(x)}{h_{K}^{2}} \leq \gamma .
$$

Note that this implies $\left\|F_{K}^{\prime}\right\|_{L^{\infty}(\widehat{K})} \leq C h_{K}$.

(4) The triangulation is regular: The intersection $\bar{K}_{1} \cap \bar{K}_{2}$ of two elements $K_{1} \neq K_{2} \in \mathcal{T}$ is either empty, exactly one vertex or exactly one edge (including its two endpoints). If the intersection $\bar{K}_{1} \cap \bar{K}_{2}$ is an edge $e=F_{K_{1}}\left(\widehat{e}_{1}\right)=F_{K_{2}}\left(\widehat{e}_{2}\right)$ (for two edges $\widehat{e}_{1}$, $\widehat{e}_{2}$ of the reference element $\widehat{K}$ ), then $\left.F_{K_{1}}^{-1} \circ F_{K_{2}}\right|_{\hat{e}_{1}}: \widehat{e}_{1} \rightarrow \widehat{e}_{2}$ is an affine bijection.

(5) Each edge is either fully contained in $\Gamma$ or part of $\partial \Gamma$.

A crucial role in our construction will be played by the elements sharing a vertex or an edge. We introduce the following definition.

Definition 2.2. Denote the set of all vertices by $\mathcal{V}$ and the set of all edges by $\mathcal{E}$. For a vertex $V \in \mathcal{V}$ and an edge $e \in \mathcal{E}$ we denote the vertex and edge patches by

$$
\omega_{V}:=\operatorname{interior}\left(\bigcup_{K \in \mathcal{T}: V \in \bar{K}} \bar{K}\right), \quad \omega_{e}:=\operatorname{interior}\left(\bigcup_{K \in \mathcal{T}: e \subset \bar{K}} \bar{K}\right) \text {. }
$$


For each patch denote the local mesh size as $h_{V}:=\operatorname{diam}\left(\omega_{V}\right)$ and $h_{e}:=\operatorname{diam}\left(\omega_{e}\right)$. The set of vertices $\mathcal{V}$ of the triangulation $\mathcal{T}$ is decomposed as $\mathcal{V}=\mathcal{V}^{\text {int }} \dot{\mathcal{V}} \mathcal{V}^{b d y}$, where $\mathcal{V}^{\text {int }}=\{z \in \mathcal{V}: z \in \Gamma\}$ and $\mathcal{V}^{b d y}=\{z \in \mathcal{V}: z \in \partial \Gamma\}$. Analogously we decompose the edges as $\mathcal{E}=\mathcal{E}^{\text {int }} \dot{\cup} \mathcal{E}^{b d y}$.

We are interested in the space of piecewise polynomials on the triangulation. For $p \in \mathbb{N}$, we denote by $\mathcal{P}_{p}:=\operatorname{span}\left\{x^{i} y^{j}: 0 \leq i, j \leq p \wedge i+j \leq p\right\}$ the space of polynomials of (total) degree $p$ and by $\mathcal{Q}_{p}:=\operatorname{span}\left\{x^{i} y^{j}: 0 \leq i, j \leq p\right\}$ the tensor-product space of polynomials of degree $p$ in each variable. We write

$$
P^{p}(\widehat{K}):= \begin{cases}\mathcal{P}_{p} & \text { if } \widehat{K}=\widehat{T} \\ \mathcal{Q}_{p} & \text { if } \widehat{K}=\widehat{S}\end{cases}
$$

If we want to emphasize the domain under consideration, we write $\mathcal{P}_{p}(\widehat{K})$ and $\mathcal{Q}_{p}(\widehat{K})$ for $\widehat{K} \in\{\widehat{S}, \widehat{T}\}$ for the two different polynomial spaces. For each element $K \in \mathcal{T}$ we choose a polynomial degree $p_{K} \in \mathbb{N}$ and collect them in the family $\mathbf{p}:=\left(p_{K}\right)_{K \in \mathcal{T}}$. We define the space of piecewise polynomials as:

$$
\mathcal{S}^{\mathbf{p}, 0}(\mathcal{T}):=\left\{u \in L^{2}(\Gamma): u \circ F_{K} \in P^{p_{K}}(\widehat{K}) \forall K \in \mathcal{T}\right\}
$$

For the discretization of the spaces $H^{\theta}(\Gamma)$ and $\widetilde{H}^{\theta}(\Gamma)$ we consider spaces of globally continuous piecewise polynomials:

$$
\begin{aligned}
& \mathcal{S}^{\mathbf{p}, 1}(\mathcal{T}):=\mathcal{S}^{\mathbf{p}, 0}(\mathcal{T}) \cap H^{1}(\Gamma), \\
& \widetilde{\mathcal{S}}^{\mathbf{p}, 1}(\mathcal{T}):=\mathcal{S}^{\mathbf{p}, 1}(\mathcal{T}) \cap \widetilde{H}^{1}(\Gamma)=\left\{u \in \mathcal{S}^{\mathbf{p}, 1}(\mathcal{T}):\left.u\right|_{\partial \Gamma}=0\right\}
\end{aligned}
$$

For subtriangulations $\left.\mathcal{T}\right|_{\omega}$ we define $\widetilde{\mathcal{S}}^{\mathbf{p}, 1}\left(\left.\mathcal{T}\right|_{\omega}\right)$ analogously, i.e., globally continuous piecewise polynomials that vanish on $\partial \omega$. We introduce the piecewise constant local mesh size function $h \in \mathcal{S}^{0,0}(\mathcal{T})$ as the function satisfying $\left.h\right|_{K}:=\operatorname{diam}(K)$ for all $K \in \mathcal{T}$ and the polynomial degree distribution $p \in \mathcal{S}^{0,0}(\mathcal{T})$ as $\left.p\right|_{K}:=p_{K}$, for all $K \in \mathcal{T}$. For a set $M \subset \mathbb{R}^{d}$, we denote by $d_{M}(\cdot):=\operatorname{dist}(\cdot, M)$ the distance to the set $M$.

For some results, we rely on the following assumption regarding the polynomial degree distribution wherever triangles and quadrilaterals meet:

Assumption 2.3. If a triangle $T$ and a quadrilateral $S$ of the triangulation $\mathcal{T}$ share an edge $e$, the corresponding polynomial degrees $p_{T}$ and $p_{S}$ satisfy

$$
p_{T} \leq p_{S} \quad \text { or } \quad p_{S} \leq 2 p_{T} .
$$

\subsection{Main results}

The main result of this paper, which underlies the stability of the additive Schwarz preconditioner discussed in Section 3.2 ahead, states that we can decompose the space $\widetilde{\mathcal{S}}^{\mathbf{p}, 1}(\mathcal{T})$ into local contributions in an $H^{\theta}$-stable way. To that end, define the spaces

$$
\begin{aligned}
X_{0} & =\prod_{V \in \mathcal{V}^{\text {int }}} L^{2}\left(\omega_{V}\right), & X_{1} & =\prod_{V \in \mathcal{V}^{\text {int }}} \widetilde{H}_{h}^{1}\left(\omega_{V}\right), \\
Y_{0} & =\prod_{e \in \mathcal{E}^{\text {int }}} L^{2}\left(\omega_{e}\right), & Y_{1} & =\prod_{V \in \mathcal{E}^{\text {int }}} \widetilde{H}_{h}^{1}\left(\omega_{e}\right), \\
Z_{0} & =\prod_{K \in \mathcal{T}} L^{2}(K), & Z_{1} & =\prod_{K \in \mathcal{T}} \widetilde{H}_{h}^{1}(K),
\end{aligned}
$$

equipped with norms and seminorms as described in Section 2.1.

The following theorem has in a similar form already appeared in Heu99 for rectangular meshes and was presented for triangulations in [FMPR15, Lemma 5.2]. 
Theorem 2.4. For $\theta \in(0,1)$, there exists a constant $C_{\theta}$ that depends only on $\Gamma, \Omega$, the $\gamma$-shape regularity of the triangulation $\mathcal{T}$, and $\theta$, such that for all $u \in \widetilde{H}^{\theta}(\Gamma)$, and for all decompositions

$$
u=u_{1}+\sum_{V \in \mathcal{V}^{i n t}} u_{V}+\sum_{e \in \mathcal{E}^{i n t}} u_{e}+\sum_{K \in \mathcal{T}} u_{K}
$$

with $\operatorname{supp} u_{V} \subseteq \omega_{V}, \operatorname{supp} u_{e} \subseteq \omega_{e}$ and $\operatorname{supp} u_{K} \subseteq K$, we can estimate:

$$
\begin{aligned}
\|u\|_{\widetilde{H}^{\theta}(\Gamma)}^{2} & \leq C_{\theta}\left(\left\|u_{1}\right\|_{\widetilde{H}^{\theta}(\Gamma)}^{2}+\sum_{V \in \mathcal{V}^{i n t}}\left\|u_{V}\right\|_{\widetilde{H}^{\theta}(\Gamma)}^{2}+\sum_{e \in \mathcal{E}^{i n t}}\left\|u_{e}\right\|_{\widetilde{H}^{\theta}(\Gamma)}^{2}+\sum_{K \in \mathcal{T}}\left\|u_{K}\right\|_{\widetilde{H}^{\theta}(\Gamma)}^{2}\right) \\
& \leq C_{\theta}\left(\left\|u_{1}\right\|_{\widetilde{H}^{\theta}(\Gamma)}^{2}+\sum_{V \in \mathcal{V}^{i n t}}\left\|u_{V}\right\|_{\widetilde{H}_{h}^{\theta}\left(\omega_{V}\right)}^{2}+\sum_{e \in \mathcal{E}^{i n t}}\left\|u_{e}\right\|_{\widetilde{H}_{h}^{\theta}\left(\omega_{e}\right)}^{2}+\sum_{K \in \mathcal{T}}\left\|u_{K}\right\|_{\widetilde{H}_{h}^{\theta}(K)}^{2}\right) .
\end{aligned}
$$

Proof. The proof is based on a so called coloring argument, we include it for completeness. The main ingredient is the following estimate (see [SS11, Lemma 4.1.49] or [vP89, Lemma 3.2]): Let $w_{j}, j=1, \ldots, n$ be functions in $\widetilde{H}^{\theta}(\Gamma)$ for $\theta \geq 0$ with pairwise disjoint support. Then it holds

$$
\left\|\sum_{i=1}^{n} w_{i}\right\|_{\widetilde{H}^{\theta}(\Gamma)}^{2} \lesssim \sum_{i=1}^{n}\left\|w_{i}\right\|_{\widetilde{H}^{\theta}(\Gamma)}^{2} .
$$

(The implied constant is known for the case of using the Aaronstein-Slobodeckij norm to be 5/2, SS11, Lemma 4.1.49]). For notational simplicity we only consider the vertex contributions, the edge and inner parts are treated in exactly the same way. By $\gamma$-shape regularity, the number of vertices in a patch can be uniformly bounded by some constant $N_{c}$. Thus, we can sort the vertices into sets $J_{1}, \ldots, J_{N_{c}}$, such that $\bigcup_{i=1}^{N_{c}} J_{i}=\mathcal{V}^{\text {int }}$ and $\left|\omega_{V} \cap \omega_{V^{\prime}}\right|=0$ for all $V, V^{\prime}$ in the same index set $J_{i}$. Repeated application of the triangle inequality and (2.10) then gives:

$$
\begin{aligned}
\|u\|_{\widetilde{H}^{\theta}(\Gamma)}^{2} & \leq 2\left\|u_{1}\right\|_{\widetilde{H}^{\theta}(\Gamma)}^{2}+2\left\|\sum_{V \in \mathcal{V}^{i n t}} u_{V}\right\|_{\widetilde{H}^{\theta}(\Gamma)}^{2} \leq 2\left\|u_{1}\right\|_{\widetilde{H}^{\theta}(\Gamma)}^{2}+2 N_{c} \sum_{i=1}^{N_{c}}\left\|\sum_{V \in J_{i}} u_{V}\right\|_{\widetilde{H}^{\theta}(\Gamma)}^{2} \\
& \leq 2\left\|u_{1}\right\|_{\widetilde{H}^{1 / 2}(\Gamma)}^{2}+2 N_{c} C \sum_{V \in \mathcal{V}^{\text {int }}}\left\|u_{V}\right\|_{\widetilde{H}^{\theta}(\Gamma)}^{2} .
\end{aligned}
$$

To prove the second estimate, see Remark 4.2 .

The primary objective of the present work is to provide the following converse estimate:

Theorem 2.5 (stable decomposition of $\widetilde{H}^{\theta}(\Gamma)$-continuous and discrete). (i) Any function $u \in L^{2}(\Gamma)$ can be written in the form

$$
u=u_{1}+\sum_{V \in \mathcal{V}^{i n t}} u_{V}+\sum_{e \in \mathcal{E}^{i n t}} u_{e}+\sum_{K \in \mathcal{T}} u_{K}
$$

where the components $u_{1} \in \widetilde{\mathcal{S}}^{1,1}(\mathcal{T}),\left(u_{V}\right)_{V \in \mathcal{V}^{\text {int }}} \subset X_{0},\left(u_{e}\right)_{e \in \mathcal{E}^{\text {int }}} \subset Y_{0}$, and $\left(u_{K}\right)_{K \in \mathcal{T}} \subset Z_{0}$ of the decomposition (2.11) depend linearly on $u$, and the corresponding linear maps have the following mapping properties:

$$
\begin{aligned}
& u \mapsto u_{1} \quad: \quad L^{2}(\Gamma) \rightarrow\left(\widetilde{\mathcal{S}}^{1,1}(\mathcal{T}),\|\cdot\|_{L^{2}}\right), \quad \widetilde{H}^{1}(\Gamma) \rightarrow\left(\widetilde{\mathcal{S}}^{1,1}(\mathcal{T}),\|\cdot\|_{H^{1}}\right), \\
& u \mapsto\left(u_{V}\right)_{V}: \quad L^{2}(\Gamma) \rightarrow X_{0}, \quad \widetilde{H}^{1}(\Gamma) \rightarrow X_{1}, \\
& u \mapsto\left(u_{e}\right)_{e}: \quad L^{2}(\Gamma) \rightarrow Y_{0}, \quad \widetilde{H}^{1}(\Gamma) \rightarrow Y_{1}, \\
& u \mapsto\left(u_{K}\right)_{K}: \quad L^{2}(\Gamma) \rightarrow Z_{0}, \quad \widetilde{H}^{1}(\Gamma) \rightarrow Z_{1} .
\end{aligned}
$$


The constants of these bounded linear maps depend solely on $\Gamma, \Omega$, and the shape regularity constant of $\mathcal{T}$. Additionally, for every $\theta \in(0,1)$ there exist constants $C_{\theta}$ (depending solely on $\theta, \Gamma, \Omega$, and the shape regularity) such that:

$$
\begin{array}{r}
\left\|u_{1}\right\|_{\widetilde{H}^{\theta}(\Gamma)}^{2} \leq C_{\theta}\|u\|_{\widetilde{H}^{\theta}(\Gamma)}^{2}, \\
\sum_{V \in \mathcal{V}^{\text {int }}}\left|u_{V}\right|_{\widetilde{H}_{h}^{\theta}\left(\omega_{V}\right)}^{2}+h_{V}^{-2 \theta}\left\|u_{V}\right\|_{L^{2}\left(\omega_{V}\right)}^{2} \leq C_{\theta}\|u\|_{\widetilde{H}^{\theta}(\Gamma)}^{2}, \\
\sum_{e \in \mathcal{E}^{i n t}}\left|u_{e}\right|_{\widetilde{H}_{h}^{\theta}\left(\omega_{e}\right)}^{2}+h_{e}^{-2 \theta}\left\|u_{e}\right\|_{L^{2}\left(\omega_{e}\right)}^{2} \leq C_{\theta}\|u\|_{\widetilde{H}^{\theta}(\Gamma)}^{2}, \\
\sum_{K \in \mathcal{T}}\left|u_{K}\right|_{\widetilde{H}_{h}^{\theta}(K)}^{2}+h_{K}^{-2 \theta}\left\|u_{K}\right\|_{L^{2}(K)}^{2} \leq C_{\theta}\|u\|_{\widetilde{H}^{\theta}(\Gamma)}^{2}
\end{array}
$$

(ii) If $\mathcal{T}$ consists of triangles only and if $u \in \widetilde{\mathcal{S}}^{\mathbf{p}, 1}(\mathcal{T})$ then each of the contributions $u_{V}, u_{e}, u_{K}$ is in $\widetilde{\mathcal{S}}^{\mathbf{p}, 1}(\mathcal{T})$.

(iii) If $\mathcal{T}$ consists of triangles and quadrilaterals and if $u \in \widetilde{\mathcal{S}}^{\mathbf{p}, 1}(\mathcal{T})$, then each of the contributions $u_{V}, u_{e}$, $u_{K}$ is in $\widetilde{\mathcal{S}}^{2 \mathbf{p}, 1}(\mathcal{T})$.

We note that the decomposition of Theorem 2.5. (iii) in the general case of meshes containing both triangles and quadrilaterals does not ensure that the contributions are in $\widetilde{\mathcal{S}}^{\mathbf{p}, 1}(\mathcal{T})$. This makes Theorem 2.5 not directly applicable for the analysis of additive Schwarz methods on meshes consisting of triangles and quadrilaterals. A modification, which, however, relies on the function $u$ to be discrete, rectifies this deficiency:

Theorem 2.6 (stable localization of $\widetilde{\mathcal{S}}^{\mathbf{p}, 1}(\mathcal{T})$ ). If Assumption 2.3 holds, any function $u \in \widetilde{\mathcal{S}}^{\mathbf{p}, 1}(\mathcal{T})$ can be decomposed in the way described in Theorem [2.5 with the additional feature that the contributions $u_{V}, u_{e}$, $u_{K}$ are in $\widetilde{\mathcal{S}}^{\mathbf{p}, 1}(\mathcal{T})$. If Assumption 2.3 is not satisfied, the stability estimates only hold for $\theta \in\{0,1\}$.

Theorem 2.7 (stable decomposition of $H^{\theta}(\Gamma)$ - continuous and discrete). The statements of Theorems 2.5, 2.6 are also true in the spaces $H^{\theta}(\Gamma)$ instead of $\widetilde{H}^{\theta}(\Gamma)$. Then, the sums run over all vertices/edges instead of just the interior ones.

Remark 2.8. While one can prove Theorem 2.5 by first considering the cases $\theta=0$ and $\theta=1$ and then using an interpolation argument, the proof of Theorem 2.6 is more involved due to the fact that mixed meshes are considered. Indeed, the decomposition operators that do not increase the polynomial degrees for quadrilaterals are only stable when applied to (piecewise) polynomial functions (due to the Gauss-Lobatto interpolation step). Performing the interpolation step at the end would therefore require a stability estimate of the form

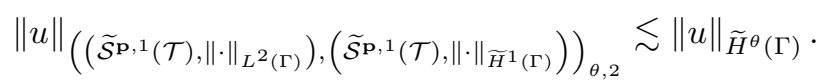

Such estimates are not available in the required generality (in fact, the present decomposition result forms the basis for a proof of such estimates). Therefore we cannot rely on performing a single interpolation step at the end. Instead we carefully work with fractional Sobolev norms throughout our proof, making sure to perform interpolation arguments only on the reference element where the necessary norm equivalences are known (see Proposition 5.6).

\section{Applications}

\subsection{Interpolation of discrete $L^{2}$ spaces with weights}

Interpolation of piecewise polynomial spaces equipped with weighted $L^{2}$ norms is common when working with a non-uniform triangulation or non-constant polynomial degree, and appears, e.g., in the context of inverse estimates, Geo08. Theorem 3.3 below provides a general setting for such applications.

It is well-known that the interpolation of $L^{2}$ spaces with different weights gives the $L^{2}$ space with corresponding interpolated weight: 
Proposition 3.1 ( Tar07, Lemma 23.1]). Let $w_{0}, w_{1}$ be positive, measurable functions, and let $\omega \subseteq \Gamma$ be open. Let $X_{0}:=L^{2}\left(\omega ; w_{0}\right)$ and $X_{1}:=L^{2}\left(\omega ; w_{1}\right)$ be the weighted Lebesgue spaces with norm $\|u\|_{L^{2}\left(\omega ; w_{i}\right)}=$ $\left(\int_{\omega}|u(x)|^{2} \omega_{i} d x\right)^{1 / 2}$. For $\theta \in(0,1)$ we can identify the interpolation space as

$$
\left(X_{0}, X_{1}\right)_{\theta, 2}=L^{2}\left(\omega ; w_{0}^{1-\theta} w_{1}^{\theta}\right) \quad \text { (equivalent norms). }
$$

The implied constants are explicitly known and depend only on $\theta$.

In order to formulate the analog of Proposition 3.1 for discrete spaces, we need the following definition:

Definition 3.2 (locally comparable). We call a measurable function $w: \Gamma \rightarrow \mathbb{R}$ a locally comparable weight, if $w(x)>0$ (almost) everywhere, and if there exists a constant $C_{w}>0$ such that for all vertex patches $\omega_{V}$ the following estimate holds

$$
\inf _{x \in \omega_{V}} w(x) \leq \sup _{x \in \omega_{V}} w(x) \leq C_{w} \inf _{x \in \omega_{V}} w(x) .
$$

Theorem 3.3. Let $\mathcal{T}$ satisfy Assumption 2.1, Let $w_{0}, w_{1} \in L^{\infty}(\Gamma)$ be locally comparable weights. Then we can identify the interpolation space by

$$
\begin{gathered}
\left(\left(\widetilde{\mathcal{S}}^{\mathbf{p}, 1}(\mathcal{T}),\|\cdot\|_{L^{2}\left(\Gamma ; w_{0}\right)}\right),\left(\widetilde{\mathcal{S}}^{\mathbf{p}, 1}(\mathcal{T}),\|\cdot\|_{L^{2}\left(\Gamma ; w_{1}\right)}\right)\right)_{\theta, 2}=\left(\widetilde{\mathcal{S}}^{\mathbf{p}, 1}(\mathcal{T}),\|\cdot\|_{L^{2}\left(\Gamma ; w_{0}^{1-\theta} w_{1}^{\theta}\right)}\right), \\
\left(\left(\mathcal{S}^{\mathbf{p}, 1}(\mathcal{T}),\|\cdot\|_{L^{2}\left(\Gamma ; w_{0}\right)}\right),\left(\mathcal{S}^{\mathbf{p}, 1}(\mathcal{T}),\|\cdot\|_{L^{2}\left(\Gamma ; w_{1}\right)}\right)\right)_{\theta, 2}=\left(\mathcal{S}^{\mathbf{p}, 1}(\mathcal{T}),\|\cdot\|_{L^{2}\left(\Gamma ; w_{0}^{1-\theta} w_{1}^{\theta}\right)}\right),
\end{gathered}
$$

where the norm equivalence constants depend only on $\Gamma, \Omega, \theta$, the shape regularity of $\mathcal{T}$, and $C_{w}$ from (3.1). Proof. We will only show (3.2). Let $u \in \widetilde{\mathcal{S}}^{\mathbf{p}, 1}(\mathcal{T})$. We denote by $\|\cdot\|_{\theta}$ the interpolation norm of the space

$$
\left(\left(\widetilde{\mathcal{S}}^{\mathbf{p}, 1}(\mathcal{T}),\|\cdot\|_{L^{2}\left(\Gamma, w_{0}\right)}\right),\left(\widetilde{\mathcal{S}}^{\mathbf{p}, 1}(\mathcal{T}),\|\cdot\|_{L^{2}\left(\Gamma, w_{1}\right)}\right)\right)_{\theta, 2} .
$$

The weighted $L^{2}$ norm will be denoted by $\|u\|_{L_{\theta}^{2}}:=\|u\|_{L^{2}\left(\Gamma, w_{0}^{1-\theta} w_{1}^{\theta}\right)}$. By Proposition 3.1 and the definition of the $K$-functional as an infimum we have the trivial estimate

$$
\|u\|_{\theta} \gtrsim\|u\|_{L_{\theta}^{2}} .
$$

It remains to show the converse estimate. We proceed similarly to the proof of [Tar07, Lemma 23.1].

Let $u_{1},\left(u_{V}\right)_{V \in \mathcal{V}^{\text {int }}}$ denote the decomposition of Theorem 2.6 (for simplicity of notation we assume that the edge and element contributions are included in the vertex functions).

By the local definition of the Scott-Zhang operator, it easy to see that it is also stable in weighted $L^{2}$ norms (with stability constant depending additionally on the constant $C_{w}$ of Def. 3.2). Since $u_{1}$ is constructed using this operator (see the Section 6), we have by interpolation

$$
\left\|u_{1}\right\|_{\theta} \lesssim\|u\|_{L_{\theta}^{2}}
$$

For $V \in \mathcal{V}^{\text {int }}$ and $j=0,1$ set $\left(\bar{w}_{j}\right)_{V}:=\frac{1}{2}\left(\inf _{x \in \omega_{V}} w_{j}(x)+\sup _{x \in \omega_{V}} w_{j}(x)\right)$. Since $w_{0}, w_{1}$ are locally comparable weights, we have on each patch $\left(\overline{w_{j}}\right)_{V} \sim w_{j}$. In order to estimate the infimum in the $K$ functional, we set:

$$
v(x):=\sum_{V \in \mathcal{V}^{\text {int }}} \frac{\left(\overline{w_{0}}\right)_{V}}{\left(\overline{w_{0}}\right)_{V}+t^{2}\left(\overline{w_{1}}\right)_{V}} u_{V}(x) \quad \text { and } \quad u-u_{1}-v=\sum_{V \in \mathcal{V}^{\text {int }}} \frac{t^{2}\left(\overline{w_{1}}\right)_{V}}{\left(\overline{w_{0}}\right)_{V}+t^{2}\left(\overline{w_{1}}\right)_{V}} u_{V} .
$$

Since the coefficients are independent of $x$ we have $v \in \widetilde{\mathcal{S}}^{\mathbf{p}, 1}(\mathcal{T})$ and thus $u=(u-v)+v$ is an admissible decomposition for the infimum of (2.3). Using $\left(\overline{w_{j}}\right)_{V} \sim w_{j}$ we estimate

$$
|v(x)| \lesssim \frac{w_{0}(x)}{w_{0}(x)+t^{2} w_{1}(x)} \sum_{V \in \mathcal{V}^{\text {int }}}\left|u_{V}(x)\right| \text { and }\left|u-u_{1}-v\right| \lesssim \frac{t^{2} w_{1}(x)}{w_{0}(x)+t^{2} w_{1}(x)} \sum_{V \in \mathcal{V}^{\text {int }}}\left|u_{V}\right|,
$$


where the implied constant only depends on the constant in (3.1). A simple calculation then shows

$$
K^{2}\left(t ; u-u_{1}\right) \lesssim \int_{\Gamma} \frac{t^{2} w_{0}(x) w_{1}(x)}{w_{0}(x)+t^{2} w_{1}(x)}\left(\sum_{V \in \mathcal{V}^{\text {int }}}\left|u_{V}(x)\right|\right)^{2} d x
$$

For the interpolation norm we then get by using Fubini's theorem and the substitution $t=s \sqrt{\frac{w_{0}(x)}{w_{1}(x)}}$ :

$$
\begin{aligned}
\left\|u-u_{1}\right\|_{\theta}^{2} & \lesssim \int_{0}^{\infty} \int_{\Gamma} t^{-2 \theta} \frac{t^{2} w_{0}(x) w_{1}(x)}{w_{0}(x)+t^{2} w_{1}(x)}\left(\sum_{V_{\in \mathcal{V}^{\text {int }}}}\left|u_{V}(x)\right|\right)^{2} d x \frac{d t}{t} \\
& \lesssim \int_{\Gamma} w_{0}(x)^{1-\theta} w_{1}(x)^{\theta}\left(\sum_{V \in \mathcal{V}^{\text {int }}}\left|u_{V}(x)\right|\right)^{2} \int_{0}^{\infty} \frac{s^{1-2 \theta}}{1+s^{2}} d s d x \\
& \lesssim \int_{\Gamma} w_{0}(x)^{1-\theta} w_{1}(x)^{\theta}\left(\sum_{V \in \mathcal{V}^{\text {int }}}\left|u_{V}(x)\right|\right)^{2} d x .
\end{aligned}
$$

Since each function $u_{V}$ is supported by the single patch $\omega_{V}$, and since the patches only have finite overlap, a simple coloring argument implies

$$
\left\|u-u_{1}\right\|_{\theta}^{2} \lesssim \sum_{V \in \mathcal{V}^{\text {int }}} \int_{\Gamma} w_{0}(x)^{1-\theta} w_{1}(x)^{\theta}\left|u_{V}(x)\right|^{2} d x=\sum_{V \in \mathcal{V}^{\text {int }}}\left\|u_{V}\right\|_{L_{\theta}^{2}}^{2}
$$

We observe that, since the decomposition $\left(u_{V}\right)_{V \in \mathcal{V}^{\text {int }}}$ was constructed in a local manner and $\omega_{0}^{1-\theta} \omega_{1}^{\theta}$ is locally comparable, we can exchange the $L^{2}$-norm in the stability result of Theorem 2.5 by the $L_{\theta}^{2}$ norm, which then concludes the proof.

\subsection{Additive Schwarz preconditioning for the $p$-BEM}

In this section we apply the decomposition results of Theorems 2.4, 2.5 to the $h p$-version of the boundary element method. Our model problem is the hypersingular integral operator $D$ for the Laplacian; for a more detailed discussion of boundary integral operators and their discretizations, we refer the reader to the monographs [SS11, Ste08, HW08, McL00. We note that the following setting covers both, the case of closed surfaces and screen problems. This is because in the case of closed surfaces we have $\widetilde{H}^{1 / 2}(\Gamma)=H^{1 / 2}(\Gamma)$ and $\widetilde{\mathcal{S}}^{\mathbf{p}, 1}(\mathcal{T})=\mathcal{S}^{\mathbf{p}, 1}(\mathcal{T})$.

The hypersingular integral operator $D: \widetilde{H}^{1 / 2}(\Gamma) \rightarrow H^{-1 / 2}(\Gamma)$ is defined by

$$
(D u)(x):=-\partial_{n_{x}}^{i n t} \int_{\Gamma} \partial_{n_{y}}^{i n t} G(x, y) u(y) d S_{y}, \quad \text { for } x \in \Gamma,
$$

where $G(x, y):=\frac{1}{4 \pi} \frac{1}{|x-y|}$ is the fundamental solution of the 3D-Laplacian and $\partial_{n_{x}}^{\text {int }}$ denotes the (interior) normal derivative with respect to $x$.

In the case of a closed, connected surface, the kernel of $D$ consists of the constant functions. In order to get a well-posed system it is customary to introduce, for some chosen $\alpha>0$, the stabilized form

$$
\langle\widetilde{D} u, v\rangle_{\Gamma}:=\langle D u, v\rangle_{\Gamma}+\alpha\langle u, 1\rangle_{\Gamma}\langle v, 1\rangle_{\Gamma} \quad \forall u, v \in \widetilde{H}^{1 / 2}(\Gamma),
$$

where $\langle\cdot, \cdot\rangle_{\Gamma}$ denotes the extension of the standard $L^{2}$-inner product to $H^{-1 / 2}(\Gamma) \times \widetilde{H}^{1 / 2}(\Gamma)$. This bilinear form is known to be bounded and elliptic, i.e., there exist some constants $c, C>0$ such that $\left|\langle\widetilde{D} u, v\rangle_{\Gamma}\right| \leq$ $C\|u\|_{\widetilde{H}^{1 / 2}(\Gamma)}\|v\|_{\widetilde{H}^{1 / 2}(\Gamma)}$ and $\langle\widetilde{D} u, u\rangle_{\Gamma} \geq c\|u\|_{\widetilde{H}^{1 / 2}(\Gamma)}^{2}$ for all $u, v \in \widetilde{H}^{1 / 2}(\Gamma)$. In the case of an open surface $\Gamma \neq \partial \Omega$ the kernel of $D$ is trivial and already $D$ is elliptic so that we may set $\alpha=0$. 
The Galerkin matrix $\widetilde{\mathbf{D}}_{h p}$ corresponding to the Galerkin discretization of this bilinear form based on the space $\widetilde{\mathcal{S}}^{\mathbf{p}, 1}(\mathcal{T})$ with chosen basis $\left(\varphi_{i}\right)_{i}$ is given by $\left(\widetilde{\mathbf{D}}_{h p}\right)_{i j}:=\left\langle\widetilde{D} \varphi_{j}, \varphi_{i}\right\rangle_{\Gamma}$.

We will present a preconditioner for this matrix based on the abstract additive Schwarz framework that will allow for $h p$-independent bounds on the condition number of the preconditioned system. It is based on the decomposition into the vertex, edge and element patch spaces, given in (2.11). We briefly recall some important definitions of the additive Schwarz theory. For a detailed introduction see [TW05, Chapter 2].

Let $a(\cdot, \cdot): \mathfrak{V} \times \mathfrak{V} \rightarrow \mathbb{R}$ be a symmetric, positive definite bilinear form on a finite dimensional vector space $\mathfrak{V}$. We will write $\mathbf{A}$ for the corresponding Galerkin matrix. Let $\mathfrak{V}_{i} \subseteq \mathfrak{V}, i=0, \ldots, N$, be a family of subspaces and let $R_{i}^{T}: \mathfrak{V}_{i} \rightarrow \mathfrak{V}$ denote the canonical embedding operators (we will use the symbol $\mathbf{R}_{i}^{T}$ for their matrix representation). Assume that for each subspace $\mathfrak{V}_{i}$ a symmetric, positive definite bilinear form $\widetilde{a}_{i}(\cdot, \cdot)$ is given; its Galerkin matrix is denoted $\widetilde{\mathbf{A}}_{i}$. Assume the spaces $\mathfrak{V}_{i}$ form a decomposition of $\mathfrak{V}$, i.e., we can write

$$
\mathfrak{V}=R_{0}^{T} \mathfrak{V}_{0}+\sum_{i=1}^{N} R_{i}^{T} \mathfrak{V}_{i} .
$$

We then define the additive Schwarz preconditioner by:

$$
\mathbf{B}^{-1}:=\sum_{i=0}^{N} \mathbf{R}_{i}^{T} \widetilde{\mathbf{A}}_{i}^{-1} \mathbf{R}_{i} .
$$

The preconditioner induced by the decomposition defined in (2.11) is optimal in $h$ and $p$. This is formalized in the following theorem:

Theorem 3.4. Let $a(u, v)=\langle\widetilde{D} u, v\rangle_{\Gamma}$. Let $\mathfrak{V}_{0}:=\widetilde{\mathcal{S}}^{1,1}(\mathcal{T})$ be the global lowest order space and $R_{0}^{T}$ : $\mathfrak{V}_{0} \rightarrow \widetilde{\mathcal{S}}^{\mathbf{p}, 1}(\mathcal{T})$ be the canonical embedding. For every $V \in \mathcal{V}^{\text {int }}$ we define the space $\mathfrak{V}_{V}:=\left\{u \in \widetilde{\mathcal{S}}^{\mathbf{p}, 1}(\mathcal{T})\right.$ : $\left.\operatorname{supp}(u) \subseteq \bar{\omega}_{V}\right\}$ with canonical embedding $R_{V}^{T}: \mathfrak{V}_{V} \rightarrow \widetilde{\mathcal{S}}^{\mathbf{p}}, 1(\mathcal{T})$. We use "exact local solvers", i.e., $\widetilde{a}_{V}(u, v):=$ $\left\langle\widetilde{D} R_{V}^{T} u, R_{V}^{T} v\right\rangle_{\Gamma}$ with matrix representation $\widetilde{\mathbf{A}}_{V}$. Let Assumption 2.3 hold. Then the preconditioner defined by

$$
\mathbf{B}^{-1}:=\mathbf{R}_{0}^{T} \mathbf{A}_{0}^{-1} \mathbf{R}_{0}+\sum_{V \in \mathcal{V}^{\text {int }}} \mathbf{R}_{V}^{T} \widetilde{\mathbf{A}}_{V}^{-1} \mathbf{R}_{V}
$$

is optimal in the sense that there exists a constant $C>0$ that only depends only on $\Gamma, \Omega$, and the $\gamma$-shape regularity of $\mathcal{T}$ such that

$$
\kappa\left(\mathbf{B}^{-1} \widetilde{\mathbf{D}}_{h p}\right):=\frac{\lambda_{\max }\left(\mathbf{B}^{-1} \widetilde{\mathbf{D}}_{h p}\right)}{\lambda_{\min }\left(\mathbf{B}^{-1} \widetilde{\mathbf{D}}_{h p}\right)} \leq C .
$$

Proof. The abstract additive Schwarz theory gives the condition number estimate $\kappa \leq C_{0} C_{1}$ in terms of the following two constants $C_{0}, C_{1}$ (see [Zha92, Lio88, MN85]):

(i) The constant $C_{0}>0$ is such that every $u \in \mathfrak{V}$ admits a decomposition $u=\sum_{i=0}^{J} R_{i}^{T} u_{i}$ with $u_{i} \in \mathfrak{V}_{i}$ such that $\sum_{i=0}^{J} \widetilde{a}_{i}\left(u_{i}, u_{i}\right) \leq C_{0} a(u, u)$.

(ii) The constant $C_{1}>0$ is such that for every decomposition $u=\sum_{i=0}^{J} R_{i}^{T} v_{i}$ with $v_{i} \in \mathfrak{V}_{i}$ the following estimate holds: $a(u, u) \leq C_{1} \sum_{i=0}^{J} \widetilde{a}_{i}\left(v_{i}, v_{i}\right)$.

Since $\widetilde{D}$ is continuous and elliptic, we can replace $a(\cdot, \cdot)$ with the $\widetilde{H}^{1 / 2}(\Gamma)$-norm. The requirement (ii) then corresponds to the stability statement of Theorem 2.6 (where we absorbed the element and edge contributions into the vertex parts) in conjunction with Remark 4.2. The requirement (iii) is just an application of Theorem 2.4

We refer to [FMPR15] for studies concerning the numerical performance of the preconditioner of Theorem 3.4 
Remark 3.5. The preconditioner of Theorem 3.4 requires a solver for the space $\mathfrak{V}_{0}=\widetilde{\mathcal{S}}^{1,1}(\mathcal{T})$ of piecewise linears. This solver can be replaced with a multilevel method as advocated, e.g., in [TS96, AM03, FMPR15.

\section{Interpolation of SOBOlev nORMS}

\subsection{Interpolation of Sobolev norms and seminorms}

As is well-known, the $L^{2}$-norm and the $H^{1}$-seminorm scale differently under affine changes of variables, and the full $H^{1}$-norm does not have a natural scaling property. Thus, the effect of domain scalings on fractional Sobolev spaces, which are defined by interpolation between $L^{2}$ and $H^{1}$ is not very clear. One way to clarify the impact of domain scalings is to study the interpolation of seminorms. The following lemma works out the corresponding norm equivalences.

Lemma 4.1 (interpolation of seminorms). Let $X_{1} \subseteq X_{0}$ be two Banach spaces with norms $\|\cdot\|_{0}$ and $\|\cdot\|_{1}:=H^{-1}\|\cdot\|_{0}+|\cdot|_{1}$, where $|\cdot|_{1}$ is a seminorm and $H>0$. Introduce the following two $K$-functionals:

$$
K^{2}(u, t):=\inf _{v \in X_{1}}\|u-v\|_{0}^{2}+t^{2}\|v\|_{1}^{2}, \quad k^{2}(u, t):=\inf _{v \in X_{1}}\|u-v\|_{0}^{2}+t^{2}|v|_{1}^{2} .
$$

For $\theta \in(0,1)$ introduce the seminorm $|\cdot|_{\theta}$ and the norms $\|\cdot\|_{\theta}$ and $\|\cdot\|_{\tilde{\theta}}$ by

$$
\begin{aligned}
|u|_{\theta}^{2} & =\int_{t=0}^{\infty} t^{-2 \theta} k^{2}(u, t) \frac{d t}{t}, \\
\|u\|_{\theta}^{2} & =\int_{t=0}^{\infty} t^{-2 \theta} K^{2}(u, t) \frac{d t}{t}, \\
\|u\|_{\tilde{\theta}}^{2} & =H^{-2 \theta}\|u\|_{0}^{2}+|u|_{\theta}^{2} .
\end{aligned}
$$

Then there exists $C>0$, which depends solely on $\theta$ (in particular, it is independent of $H$ ), such that

$$
C^{-1}\|u\|_{\theta} \leq\|u\|_{\tilde{\theta}} \leq C\|u\|_{\theta} .
$$

Proof. We show $\|u\|_{\tilde{\theta}} \leq C\|u\|_{\theta}$ : Obviously, $|u|_{\theta} \leq\|u\|_{\theta}$. In order to see $H^{-\theta}\|u\|_{0} \leq C\|u\|_{\theta}$, we observe that for arbitrary $v \in X_{1}$

$$
\|u\|_{0} \leq\|u-v\|_{0}+\|v\|_{0} \leq\|u-v\|_{0}+\frac{H}{t} t\|v\|_{1},
$$

and hence $\|u\|_{0} \leq \max \{1, H / t\} K(u, t)$. This implies

$$
H^{-2 \theta}\left(\frac{1}{2 \theta}+\frac{1}{2(1-\theta)}\right)\|u\|_{0}^{2}=\int_{t=0}^{\infty} t^{-2 \theta-1} \min \{1, t / H\}^{2}\|u\|_{0}^{2} d t \leq \int_{t=0}^{\infty} t^{-2 \theta-1} K^{2}(u, t) d t=\|u\|_{\theta}^{2} .
$$

Next, we show $\|u\|_{\theta} \leq C\|u\|_{\tilde{\theta}}$. We write

$$
\|u\|_{\theta}^{2}=\int_{t=0}^{H} t^{-2 \theta-1} K^{2}(u, t) d t+\int_{t=H}^{\infty} t^{-2 \theta-1} K^{2}(u, t) d t .
$$

To treat the first integral, we let $\tilde{v}$ be the minimizer of $k^{2}(u, t)$, i.e., $\tilde{v}=\operatorname{argmin}_{\operatorname{nnf}} \operatorname{s\in X}_{1}\|u-v\|_{0}^{2}+t^{2}|v|_{1}^{2}$ (If the minimum is not attained, then select $v_{\varepsilon}$ with $\left\|u-v_{\varepsilon}\right\|_{0}^{2}+t^{2}\left|v_{\varepsilon}\right|_{1}^{2} \leq k^{2}(u, t)+\varepsilon$ and let $\varepsilon \rightarrow 0$ at the end.) Then

$$
K^{2}(u, t) \leq\|u-\tilde{v}\|_{0}^{2}+t^{2}\|\tilde{v}\|_{1}^{2} \leq\|u-\tilde{v}\|_{0}^{2}+\frac{t^{2}}{H^{2}}\|\tilde{v}\|_{0}^{2}+t^{2}|\tilde{v}|_{1}^{2} \lesssim \max \left\{1, \frac{t^{2}}{H^{2}}\right\} k^{2}(u, t)+\frac{t^{2}}{H^{2}}\|u\|_{0}^{2} .
$$

Therefore,

$$
\int_{t=0}^{H} t^{-2 \theta-1} K^{2}(u, t) d t \lesssim \int_{t=0}^{H} t^{-2 \theta-1} k^{2}(u, t) d t+H^{-2} \int_{t=0}^{H} t^{-2 \theta+1}\|u\|_{0}^{2} d t \lesssim|u|_{\theta}^{2}+H^{-2 \theta}\|u\|_{0}^{2} .
$$


To treat the second integral in (4.1), we use the obvious estimate $K(u, t) \leq\|u\|_{0}$ and see

$$
\int_{t=H}^{\infty} t^{-2 \theta-1} K^{2}(u, t) d t \leq \int_{t=H}^{\infty} t^{-2 \theta-1}\|u\|_{0}^{2} d t \leq C H^{-2 \theta}\|u\|_{0}^{2} .
$$

Therefore, $\|u\|_{\theta}^{2} \lesssim H^{-2 \theta}\|u\|_{0}^{2}+|u|_{\theta}^{2}$ with implied constants depending only on $\theta$.

Lemma 4.1 gives the following norm equivalence for the standard fractional Sobolev norms and the weighted versions:

$$
|u|_{\widetilde{H}^{\theta}(\omega)}+\operatorname{diam}(\omega)^{-\theta}\|u\|_{L^{2}(\omega)} \sim\|u\|_{\widetilde{H}_{h}^{\theta}(\omega)} \quad \text { and } \quad|u|_{H^{\theta}(\omega)}+\operatorname{diam}(\omega)^{-\theta}\|u\|_{L^{2}(\omega)} \sim\|u\|_{H_{h}^{\theta}(\omega)} .
$$

Concerning the norms on patches, we note the following:

Remark 4.2. Since for any vertex or edge patch $\omega \subset \Gamma$ the operator realizing the extension by zero is bounded with constant 1 as an operator $L^{2}(\omega) \rightarrow L^{2}(\Gamma)$ and $\widetilde{H}_{h}^{1}(\omega) \rightarrow \widetilde{H}^{1}(\Gamma)$ we obtain by interpolation

$$
\|u\|_{\widetilde{H}^{\theta}(\Gamma)} \lesssim\|u\|_{\widetilde{H}_{h}^{\theta}(\omega)} \stackrel{\sqrt{4.2} \sim}{\sim}|u|_{\widetilde{H}_{h}^{\theta}(\omega)}+\operatorname{diam}(\omega)^{-\theta}\|u\|_{L^{2}(\omega)} \quad \forall u \in \widetilde{H}^{\theta}(\omega) .
$$

Next, we study the scaling of interpolation seminorms. The following corollary can be seen as a generalization of [Heu14, Lemma 2.8] and provides the application of Lemma [4.1 to the present setting of 2D surface elements.

Corollary 4.3. Let $K \in \mathcal{T}$ be an element and $\widehat{K}$ be the associated reference element. Suppose that $X_{1} \subset$ $L^{2}(K)$ and $\widehat{X}_{1} \subset L^{2}(\widehat{K})$ are continuously embedded Banach spaces such that for every $u \in X_{1}$ and its pullback $\widehat{u}:=u \circ F_{K}$ to the reference element $\widehat{K}$ there holds $\widehat{u} \in \widehat{X}_{1}$. Let $|\cdot|_{X_{1}}$ and $|\cdot|_{\widehat{X}_{1}}$ be seminorms (or norms) on $X_{1}$ with $|u|_{X_{1}} \sim|\widehat{u}|_{\widehat{X}_{1}}$. Define $|\cdot|_{\theta, K}$ and $|\cdot|_{\theta, \widehat{K}}$ as in Lemma 4.1. where $X_{0}=L^{2}(K)$ and $\widehat{X}_{0}=L^{2}(\widehat{K})$. Then it holds for $\theta \in[0,1]$

$$
|u|_{\theta, K} \sim h_{K}^{1-\theta}|\widehat{u}|_{\theta, \widehat{K}}
$$

In particular, we have

$$
\|u\|_{\left(L^{2}(K), X_{1}\right)_{\theta, 2}} \sim h_{K}^{1-\theta}\|\widehat{u}\|_{\left(L^{2}(\widehat{K}), \widehat{X}_{1}\right)_{\theta, 2}} .
$$

Proof. Write $k^{2}(u, t)=\inf _{v \in X_{1}}\|u-v\|_{L^{2}(K)}^{2}+t^{2}|v|_{X_{1}}^{2}$. Then

$$
\|\widehat{u}-\widehat{v}\|_{L^{2}(\widehat{K})}^{2}+t^{2}|\widehat{v}|_{\widehat{X}_{1}}^{2} \sim h_{K}^{-2}\|u-v\|_{L^{2}(K)}^{2}+t^{2}|v|_{X_{1}}^{2} .
$$

Therefore,

$$
\inf _{w \in \widehat{X}_{1}}\|\widehat{u}-w\|_{L^{2}(\widehat{K})}^{2}+t^{2}|w|_{\widehat{X}_{1}}^{2} \lesssim h_{K}^{-2} k^{2}\left(u, t h_{K}\right),
$$

and thus

$$
|\widehat{u}|_{\theta, \widehat{K}}^{2} \lesssim h_{K}^{-2} \int_{t=0}^{\infty} t^{-2 \theta} k^{2}\left(u, t h_{K}\right) \frac{d t}{t} \lesssim h_{K}^{-2+2 \theta}|u|_{\theta, K}^{2}
$$

The reverse direction is shown in a similar manner. The equivalence of norms then follows with Lemma 4.1 


\subsection{Interpolation of weighted spaces}

The vertex averaging operators defined in Section 5 ahead are such that they reproduce the value in one corner of the reference element $\widehat{K}$ for functions that are continuous there. For functions with less regularity near that corner, the difference is measured in a weighted $L^{2}$-norm. Thus, weighted spaces and interpolation between weighted spaces are studied in the present section.

\subsubsection{Trace theorems and local estimates}

We recall some simple trace estimates.

Lemma 4.4. Let $\widehat{V}$ be a vertex of $\widehat{T}$ and $\widehat{e}$ an edge with $\widehat{V} \in \overline{\widehat{e}}$.

(i) Then for all $v \in H^{1}(\widehat{T})$ the following estimates hold provided that the right-hand sides are finite:

$$
\begin{aligned}
\left\|d_{\widehat{V}}^{-1} v\right\|_{L^{2}(\widehat{T})} & \lesssim\|\nabla v\|_{L^{2}(\widehat{T})}+\left\|d_{\widehat{V}}^{-1 / 2} v\right\|_{L^{2}(\widehat{e})} \\
\left\|d_{\widehat{V}}^{-1 / 2} v\right\|_{L^{2}(\widehat{e})} & \lesssim\left\|d_{\widehat{V}}^{-1} v\right\|_{L^{2}(\widehat{T})}+\|\nabla v\|_{L^{2}(\widehat{T})} .
\end{aligned}
$$

(ii) Define, for $t>0$, the slabs $\widetilde{A}^{2,1}(t):=\left\{x \in \widehat{T}: t<d_{\widehat{V}}(x)<2 t\right\}$. Then for all $v \in H^{1}(\widehat{T})$ the following estimates hold provided that the right-hand sides are finite:

$$
\begin{aligned}
\left\|d_{\widehat{V}}^{-1} v\right\|_{L^{2}\left(\widetilde{A}^{2,1}(t)\right)} & \lesssim\|\nabla v\|_{L^{2}\left(\widetilde{A}^{2,1}(t)\right)}+\left\|d_{\widehat{V}}^{-1 / 2} v\right\|_{L^{2}\left(\widehat{e} \cap \widetilde{A}^{2,1}(t)\right)}, \\
\left\|d_{\widehat{V}}^{-1 / 2} v\right\|_{L^{2}\left(\widehat{e} \cap \widetilde{A}^{2,1}(t)\right)} & \lesssim\left\|d_{\widehat{V}}^{-1} v\right\|_{L^{2}\left(\widetilde{A}^{2,1}(t)\right)}+\|\nabla v\|_{L^{2}\left(\widetilde{A}^{2,1}(t)\right)} .
\end{aligned}
$$

(iii) For $v \in H^{1}(\widehat{T})$ with $\left.v\right|_{\widehat{e}} \equiv 0$, it holds

$$
\left\|d_{\widehat{e}}^{-1} u\right\|_{L^{2}(\widehat{T})} \leq\|\nabla v\|_{L^{2}(\widehat{T})}
$$

Proof. The first estimate in (ii) follows from standard arguments and an application of Hardy's inequality: To keep the notation succinct, consider $\widehat{V}:=(0,0)$ and $\widehat{e}:=(0,1) \times\{0\}$; note that $d_{V} \sim \xi$. Then $u(\xi, \eta)=$ $u(\xi, 0)+\int_{t=0}^{\eta} \partial_{t} u(\xi, t) d t$ and therefore

$$
\int_{\widehat{T}} \xi^{-2} u^{2}(\xi, \eta) \lesssim \int_{\xi} \xi^{-1} u(\xi, 0)^{2} d \xi+\int_{\xi} \int_{\eta=0}^{\xi}\left|\xi^{-1} \int_{t=0}^{\eta} u_{t}(\xi, t) d t\right|^{2} d \eta d \xi .
$$

In the last integral, we estimate $\xi^{-1} \leq \eta^{-1}$ and apply Hardy's inequality. The second estimate in (ii) follows from local trace estimates near $\widehat{e}$ and a covering argument (Besicovitch). The estimates in (iii) follow in a similar manner using polar coordinates; alternatively, it can be shown by scaling arguments. The estimate (iii) follows along the same lines, using that $u(\xi, 0)$ vanishes.

Lemma 4.5. Let $u \in L^{\infty}(\widehat{T})$ and let $\widehat{V}$ be one of the vertices of $\widehat{T}$. Then, $\left\|d_{\widehat{V}}^{-1 / 2} u\right\|_{L^{2}(\widehat{T})} \lesssim\|u\|_{L^{\infty}(\widehat{T})}$.

Proof. For simplicity, we consider $\widehat{V}=(0,0)$ and note that $d_{\widehat{V}} \sim \xi$. We compute

$$
\left\|d_{\widehat{V}}^{-1 / 2} u\right\|_{L^{2}(\widehat{T})}^{2} \lesssim\|u\|_{L^{\infty}(\widehat{T})}^{2} \int_{0}^{1} \xi^{-1} \int_{0}^{\xi} 1 d \eta d \xi=\|u\|_{L^{\infty}(\widehat{T})}^{2} .
$$

\subsubsection{Interpolation of weighted spaces}

We start with an explicit construction of a function that essentially realizes the decomposition of the K-functional for a pair of Sobolev spaces:

Lemma 4.6. Let $\omega \subset \mathbb{R}^{d}$ be a bounded Lipschitz domain with $\operatorname{diam}(\omega) \leq C_{1}, \theta \in(0,1)$, and fix $\beta>0$. Then, for $u \in H_{h}^{\theta}(\omega)$, there is a function $w:(0, \infty) \rightarrow H_{h}^{1}(\omega)$ such that

$$
\int_{0}^{\infty} t^{-2 \theta}\left(\|u-w(t)\|_{L^{2}(\omega)}^{2}+t^{2}\|w(t)\|_{H_{h}^{1}(\omega)}^{2}\right) \frac{d t}{t} \lesssim\|u\|_{H_{h}^{\theta}(\omega)}^{2} .
$$


Additionally, for all subsets $\omega^{\prime} \subset \omega$ with $\operatorname{dist}\left(\omega^{\prime}, \partial \omega\right)>\beta t$ it holds

$$
\|w(t)\|_{L^{2}\left(\omega^{\prime}\right)} \lesssim\|u\|_{L^{2}\left(\cup_{x \in \omega^{\prime}} B_{\beta t / 2}(x)\right)} .
$$

The hidden constants depend only on $d, C_{1}, \beta$, and the Lipschitz constant of $\omega$.

If, additionally, $\widetilde{\omega} \subset \omega$ is a subset with $\operatorname{diam}(\omega) \leq C_{\text {diam }} \operatorname{diam}(\widetilde{\omega})$ for some constant $C_{\text {diam }}>0$, and $\operatorname{supp}(u) \subset \widetilde{\omega}$, then $w:(0, \infty) \rightarrow \widetilde{H}_{h}^{1}(\omega)$ and the right-hand side in (4.5) can be replaced by $\|u\|_{\widetilde{H}_{h}^{\theta}(\widetilde{\omega})}$; furthermore, (4.6) holds for all $\omega^{\prime} \subset \mathbb{R}^{d}$ with $u$ on the right implicitly extended by zero. The hidden constants depend additionally on $C_{\text {diam }}$.

Proof. Denote by $H_{h}^{1}\left(\mathbb{R}^{d}\right)$ the space $H^{1}\left(\mathbb{R}^{d}\right)$ with norm

$$
\|\cdot\|_{H_{h}^{1}\left(\mathbb{R}^{d}\right)}^{2}:=\operatorname{diam}(\omega)^{-2}\|\cdot\|_{L^{2}\left(\mathbb{R}^{d}\right)}^{2}+|\cdot|_{H^{1}\left(\mathbb{R}^{d}\right)}^{2},
$$

and by $H_{h}^{\theta}\left(\mathbb{R}^{d}\right)$ the interpolation between $L^{2}\left(\mathbb{R}^{d}\right)$ and $H_{h}^{1}\left(\mathbb{R}^{d}\right)$. From $\operatorname{diam}(\omega) \leq C_{1}$ we conclude that Stein's extension operator $E$ (see [Ste70, Sec. VI]) is bounded as $L^{2}(\omega) \rightarrow L^{2}\left(\mathbb{R}^{d}\right)$ and $H_{h}^{1}(\omega) \rightarrow H_{h}^{1}\left(\mathbb{R}^{d}\right)$, with a constant depending only on $d, C_{1}$, and the Lipschitz constant of $\omega$. Therefore, it suffices to show the existence of a function $w:(0, \infty) \rightarrow H_{h}^{1}\left(\mathbb{R}^{d}\right)$ such that

$$
\int_{0}^{\infty} t^{-2 \theta}\left(\|E u-w(t)\|_{L^{2}\left(\mathbb{R}^{d}\right)}^{2}+t^{2}\|w(t)\|_{H_{h}^{1}\left(\mathbb{R}^{d}\right)}^{2}\right) \frac{d t}{t} \lesssim\|E u\|_{H_{h}^{\theta}\left(\mathbb{R}^{d}\right)}^{2} .
$$

To that end, let $\rho \in C^{\infty}\left(\mathbb{R}^{d}\right)$ with $\operatorname{supp} \rho \subset B_{1}(0) \backslash B_{1 / 2}(0)$ be a mollifier and $\chi_{I}$ the indicator function of an interval $I$. Using the techniques from the proof of [AF03, Theorem 7.47] (implications $(a) \Rightarrow(b)$ and $(c) \Rightarrow(a))$, it follows that $w(t):=\chi_{[0, \operatorname{diam}(\omega)]}(t) \cdot\left(\rho_{\beta t / 2} \star E u\right)$ fulfills (4.7) where we used again $\operatorname{diam}(\omega) \leq C_{1}$. The estimate (4.6) is clear. If $\widetilde{\omega} \subset \omega$ is a subset as indicated and $u \in \widetilde{H}_{h}^{\theta}(\widetilde{\omega})$, then we can extend $u$ by zero to $\mathbb{R}^{d}$ instead of using the Stein extension.

The following lemma identifies the interpolation space between $L^{2}$ and a weighted $H^{1}$-space:

Lemma 4.7. Let $\theta \in(0,1), \widehat{K} \in\{\widehat{T}, \widehat{S}\}$ be the reference triangle or square, and let $\widehat{V}$ be one of its vertices. Consider the interpolation space between $L^{2}(\widehat{K})$ with the standard norm and $H^{1}\left(\widehat{K}, d_{\widehat{V}}^{-1}\right):=$ $\left\{u \in H^{1}(\widehat{K}):\left\|d_{\widehat{V}}^{-1} u\right\|_{L^{2}(\widehat{K})}<\infty\right\}$ with the norm $\|u\|_{H^{1}\left(\widehat{K}, d_{\widehat{V}}^{-1}\right)}^{2}:=\|u\|_{H^{1}(\widehat{K})}^{2}+\left\|d_{\widehat{V}}^{-1} u\right\|_{L^{2}(\widehat{K})}^{2}$. Then,

$$
\|u\|_{\left(L^{2}(\widehat{K}), H^{1}\left(\widehat{K}, d_{\widehat{V}}^{-1}\right)\right)_{\theta, 2}} \sim\|u\|_{H^{\theta}(\widehat{K})}+\left\|d_{\widehat{V}}^{-\theta} u\right\|_{L^{2}(\widehat{K})},
$$

and the implied constants depend only on $\theta$.

Proof. For notational simplicity, we consider the case $\widehat{K}=(0,1)^{2}$ and $\widehat{V}=(0,0)$; the case of triangles can be inferred from this one by reflection across an edge of the triangle. We first show the bound $\gtrsim$ in the desired equivalence. Note that for $t>0$ we have

$$
\inf _{v \in H^{1}\left(\widehat{K}, d_{\widehat{V}}^{-1}\right)}\left(\|u-v\|_{L^{2}(\widehat{K})}^{2}+t^{2}\|v\|_{H^{1}(\widehat{K})}^{2}+t^{2}\left\|d_{\widehat{V}}^{-1} v\right\|_{L^{2}(\widehat{K})}^{2}\right) \gtrsim K_{1}^{2}(u, t)+K_{2}^{2}(u, t),
$$

where $K_{1}(u, t)$ is the $K$-functional for the interpolation pair $\left(L^{2}(\widehat{K}), H^{1}(\widehat{K})\right)$ and $K_{2}$ is the functional for $\left(L^{2}(\widehat{K}), L^{2}\left(\widehat{K}, d_{\widehat{V}}^{-1}\right)\right)$. By Proposition 3.1 we have $\left(L^{2}(\widehat{K}), L^{2}\left(\widehat{K}, d_{\widehat{V}}^{-1}\right)\right)_{\theta, 2}=L^{2}\left(\widehat{K}, d_{\widehat{V}}^{-\theta}\right)$ and obtain the bound $\gtrsim$. For the reverse bound, consider $u \in H^{\theta}(\widehat{K})$ with $\left\|d_{\widehat{V}}^{-\theta} u\right\|_{L^{2}(\widehat{K})}<\infty$. We extend $u$ to $\omega:=(-1,1)^{2}$ in two steps by extending symmetrically first across the $y$ and then across the $x$-axis. The extended function is again denoted by $u$. Then, we choose $w:(0, \infty) \rightarrow H^{1}(\omega)$ according to Lemma 4.6 using $\beta:=1 / 2$ and get for $t<1 / 2$

$$
\|w(t)\|_{L^{2}\left(B_{t}(0) \cap \widehat{K}\right)} \leq\|w(t)\|_{L^{2}\left(B_{t}(0)\right)} \lesssim\|u\|_{L^{2}\left(B_{2 t}(0)\right)} \lesssim\|u\|_{L^{2}\left(B_{2 t}(0) \cap \widehat{K}\right)}
$$


and for $t \geq 1 / 2$

$$
\|w(t)\|_{L^{2}\left(B_{t}(0) \cap \widehat{K}\right)} \lesssim\|E u\|_{L^{2}\left(\mathbb{R}^{2}\right)} \lesssim\|u\|_{L^{2}(\widehat{K})}
$$

For $t>0$, let $\chi_{t} \in C_{0}^{\infty}\left(\mathbb{R}^{d}\right)$ denote a smooth cutoff function with $\chi_{t}(x)=1$ for all $x \in B_{t / 2}(0)$, supp $\chi_{t} \subseteq$ $B_{t}(0)$, and $\left\|\nabla \chi_{t}\right\|_{L^{\infty}\left(\mathbb{R}^{d}\right)} \leq C t^{-1}$ and define $\widetilde{w}(t):=\left(1-\chi_{t}\right) w(t)$. We calculate

$$
\begin{aligned}
\|u-\widetilde{w}(t)\|_{L^{2}(\widehat{K})} & \leq\|u-w(t)\|_{L^{2}(\widehat{K})}+\left\|\chi_{t} w(t)\right\|_{L^{2}(\widehat{K})} \leq\|u-w(t)\|_{L^{2}(\widehat{K})}+\|w(t)\|_{L^{2}\left(B_{t}(0) \cap \widehat{K}\right)}, \\
t\|\widetilde{w}(t)\|_{H^{1}(\widehat{K})} & \leq t\|w(t)\|_{H^{1}(\widehat{K})}+t\left\|\chi_{t} w(t)\right\|_{H^{1}(\widehat{K})} \lesssim t\|w(t)\|_{H^{1}(\widehat{K})}+\|w(t)\|_{L^{2}\left(B_{t}(0) \cap \widehat{K}\right)},
\end{aligned}
$$

and, with the notation $B_{r}^{c}(0)=\mathbb{R}^{2} \backslash B_{r}(0)$,

$$
\begin{aligned}
t\left\|d_{\widehat{V}}^{-1} \widetilde{w}(t)\right\|_{L^{2}(\widehat{K})} & \lesssim t\left\|d_{\widehat{V}}^{-1} w(t)\right\|_{L^{2}\left(B_{t / 2}^{c}(0) \cap \widehat{K}\right)} \lesssim t\left\|d_{\widehat{V}}^{-1}(u-w(t))\right\|_{L^{2}\left(B_{t / 2}^{c}(0) \cap \widehat{K}\right)}+t\left\|d_{\widehat{V}}^{-1} u\right\|_{L^{2}\left(B_{t / 2}^{c}(0) \cap \widehat{K}\right)} \\
& \lesssim\|u-w(t)\|_{L^{2}(\widehat{K})}+t\left\|d_{\widehat{V}}^{-1} u\right\|_{L^{2}\left(B_{t / 2}^{c}(0) \cap \widehat{K}\right)} .
\end{aligned}
$$

We obtain

$$
\begin{aligned}
& \|u\|_{\left(L^{2}(\widehat{K}), H^{1}\left(\widehat{K}, d_{\widehat{V}}^{-1}\right)\right)_{\theta, 2}}^{2} \\
& \lesssim \int_{0}^{\infty} t^{-2 \theta}\left(\|u-w(t)\|_{L^{2}(\widehat{K})}^{2}+t^{2}\|w(t)\|_{H^{1}(\widehat{K})}^{2}\right) \frac{d t}{t} \\
& +\int_{0}^{\infty} t^{-2 \theta}\|w(t)\|_{L^{2}\left(B_{t}(0) \cap \widehat{K}\right)}^{2} \frac{d t}{t}+\int_{0}^{\infty} t^{2-2 \theta}\left\|d_{\widehat{V}}^{-1} u\right\|_{L^{2}\left(B_{t / 2}^{c}(0) \cap \widehat{K}\right)}^{2} \frac{d t}{t} \\
& \lesssim\|u\|_{H^{\theta}(\omega)}^{2}+\int_{0}^{\infty} t^{-2 \theta}\|w(t)\|_{L^{2}\left(B_{t}(0) \cap \widehat{K}\right)}^{2} \frac{d t}{t}+\int_{0}^{\infty} t^{2-2 \theta}\left\|d_{\widehat{V}}^{-1} u\right\|_{L^{2}\left(B_{t / 2}^{c}(0) \cap \widehat{K}\right)}^{2} \frac{d t}{t},
\end{aligned}
$$

where we have used Lemma 4.6 in the last step. Since the symmetric extension is continuous in $L^{2}$ and $H^{1}$, an interpolation argument shows that $\|u\|_{H^{\theta}(\omega)} \lesssim\|u\|_{H^{\theta}(\widehat{K})}$, and it remains to bound the last two integrals by $\left\|d_{\widehat{V}}^{-\theta} u\right\|_{L^{2}(\widehat{K})}$. For the first term, we use (4.9) to bound

$$
\int_{1 / 2}^{\infty} t^{-2 \theta}\|w(t)\|_{L^{2}\left(B_{t}(0) \cap \widehat{K}\right)}^{2} \frac{d t}{t} \lesssim\|u\|_{L^{2}(\widehat{K})}^{2}
$$

We use (4.8) and polar coordinates to bound

$$
\begin{aligned}
\int_{0}^{1 / 2} t^{-2 \theta}\|w(t)\|_{L^{2}\left(B_{t}(0) \cap \widehat{K}\right)}^{2} \frac{d t}{t} & \lesssim \int_{0}^{1 / 2} t^{-2 \theta}\|u\|_{L^{2}\left(B_{2 t}(0) \cap \widehat{K}\right)}^{2} \frac{d t}{t}=\int_{0}^{1 / 2} t^{-2 \theta-1} \int_{0}^{2 t} \int_{0}^{2 \pi} \chi_{\widehat{K}}(r, \varphi) u^{2}(r, \varphi) r d \varphi d r d t \\
& \leq \int_{0}^{2 \pi} \int_{0}^{1} \chi_{\widehat{K}}(r, \varphi) u^{2}(r, \varphi) r \int_{r}^{\infty} t^{-2 \theta-1} d t d r d \varphi \\
& \lesssim \int_{0}^{2 \pi} \int_{0}^{1} \chi_{\widehat{K}}(r, \varphi) u^{2}(r, \varphi) r^{1-2 \theta} d r d \varphi \leq\left\|d_{\widehat{V}}^{-\theta} u\right\|_{L^{2}(\widehat{K})}^{2} .
\end{aligned}
$$

Finally, the second term in (4.10) can be estimated by

$$
\begin{aligned}
\int_{0}^{\infty} t^{2-2 \theta}\left\|d_{\widehat{V}}^{-1} u\right\|_{L^{2}\left(B_{t / 2}^{c}(0) \cap \widehat{K}\right)}^{2} \frac{d t}{t} & \leq \int_{0}^{\infty} \int_{\widehat{K} \cap\{|x|>t / 2\}} t^{1-2 \theta}|x|^{-2} u^{2}(x) d t=\int_{\widehat{K}}|x|^{-2} u^{2}(x) \int_{0}^{2|x|} t^{1-2 \theta} d t \\
& \lesssim \int_{\widehat{K}}|x|^{-2} u^{2}(x)|x|^{2-2 \theta}=\left\|d_{\widehat{V}}^{-\theta} u\right\|_{L^{2}(\widehat{K})}^{2} .
\end{aligned}
$$

The following lemma encapsulates a construction needed later on in the proof of Lemma 4.9. 
Lemma 4.8. Let $V \in \mathcal{V}$ and let $\omega_{V}$ denote its vertex patch. Fix one element $K \subseteq \omega_{V}$ and define the annuli

$$
A^{b, a}(t):=B_{b t}(V) \backslash \overline{B_{a t}(V)} \quad \text { for } t>0 \text { and } 0<a<b .
$$

Then there exists an open set $Z \subseteq K$ and a constant $\beta>0$ such that

$$
\bigcup_{z \in Z} \overline{B_{\beta d_{V}(z)}(z)} \cap \Gamma \subseteq K
$$

and for all $v \in H_{h}^{1}\left(\omega_{V}\right)$ there holds, provided that the right-hand side is finite,

$$
\left\|d_{V}^{-1} v\right\|_{L^{2}\left(A^{2,1}(t) \cap \omega_{V}\right)} \lesssim\left\|d_{V}^{-1} v\right\|_{L^{2}\left(A^{2,1}(t) \cap Z\right)}+\|v\|_{H_{h}^{1}\left(A^{2,1}(t) \cap \omega_{V}\right)} .
$$

Proof. We first show

$$
\left\|d_{V}^{-1} v\right\|_{L^{2}\left(A^{2,1}(t) \cap \omega_{V}\right)} \lesssim\left\|d_{V}^{-1} v\right\|_{L^{2}\left(A^{2,1}(t) \cap K\right)}+\|v\|_{H_{h}^{1}\left(A^{2,1}(t) \cap \omega_{V}\right)} .
$$

Let $K_{1}$ and $K_{2}$ be two elements of $\omega_{V}$ sharing an edge $e_{1,2}$. Applying both estimates from Lemma 4.4 (iii) and scaling arguments shows

$$
\begin{aligned}
\left\|d_{V}^{-1} v\right\|_{L^{2}\left(A^{2,1}(t) \cap K_{1}\right)} & \lesssim\left\|d_{V}^{-1 / 2} v\right\|_{L^{2}\left(A^{2,1}(t) \cap e_{1,2}\right)}+\|\nabla v\|_{L^{2}\left(A^{2,1}(t) \cap K_{1}\right)} \\
& \lesssim\left\|d_{V}^{-1} v\right\|_{L^{2}\left(A^{2,1}(t) \cap K_{2}\right)}+\|\nabla v\|_{L^{2}\left(A^{2,1}(t) \cap K_{2}\right)}+\|\nabla v\|_{L^{2}\left(A^{2,1}(t) \cap K_{1}\right)} .
\end{aligned}
$$

Taking a sequence of neighboring elements $K^{\prime}=K_{1}, \ldots, \widehat{K}_{j}=K$, this argument can be repeated $j$ times and summed up to yield (4.13).

We now construct the set $Z$. Let $\widehat{Z}$ be a cone on the reference element centered at $\widehat{V}:=(1,0)$, symmetric with respect to the diagonal of $\widehat{S}$ with an opening angle in $(0, \pi / 4)$. We note the existence of a constant $\widehat{\beta}>0$ such that for $\widehat{x} \in \widehat{Z}$ with $|\widehat{x}-\widehat{V}| \leq 1 / 4$, we have $\overline{B_{\beta d_{\widehat{V}}(\widehat{x})}(\widehat{x})} \subseteq \widehat{K}$. We set $Z:=F_{K}\left(\widehat{Z} \cap B_{1 / 4}(\widehat{V})\right)$ (where we assume that $\widehat{V}$ is mapped to $V$ ). To show (4.11), we just have to select $\beta \leq \widehat{\beta}$ sufficiently small to compensate for the Lipschitz constants of $F_{K}$ and $F_{K}^{-1}$.

The estimate (4.12) can then be shown completely analogously to (4.13): On the reference element, we can use Lemma 4.4, (iii) to reduce the estimate to an $H^{1}$ contribution and a $d_{\widehat{V}}^{-1 / 2}$-weighted integral on the boundary of $\widehat{Z}$. This can then in turn again be estimated by a weighted $L^{2}$-term and an $H^{1}$-term on $\widehat{Z}$, as appears on the right-hand side of (4.12). The restriction due to the condition $|\widehat{x}-\widehat{V}| \leq 1 / 4$ does not impact the estimate, as $d_{\widehat{V}}^{-1}$ is bounded outside of this region.

Lemma 4.9. Let $V \in \mathcal{V}$ and let $\omega_{V}$ denote its vertex patch. Fix one element $K \subseteq \omega_{V}$. Then the following estimate holds for all $\theta \in[0,1]$ and for all $u \in H_{h}^{1}\left(\omega_{V}\right)$, provided that the right-hand side is finite:

$$
\left\|d_{V}^{-\theta} u\right\|_{L^{2}\left(\omega_{V}\right)} \leq C\left[\left\|d_{V}^{-\theta} u\right\|_{L^{2}(K)}+\|u\|_{H_{h}^{\theta}\left(\omega_{V}\right)}\right] .
$$

The constant depends on $\theta$, the shape regularity constant $\gamma$, and $\Gamma$.

Proof. Let $c_{1}>0$ be such that $B_{c_{1} h_{V}}(V) \cap \Gamma$ is contained in a single chart of $\Gamma$ and $B_{c_{1} h_{V}}(V) \cap \Gamma \subseteq \omega_{V}$. This constant can be chosen to depend only on $\Gamma$ and the shape regularity. Set $\alpha_{1}:=c_{1} / 4$ and $\alpha_{2}:=c_{1} / 2$ and let $\chi$ be a cutoff function satisfying $\operatorname{supp}(\chi) \subseteq B_{\alpha_{2} h_{V}}(V)$ and $\chi \equiv 1$ on $B_{\alpha_{1} h_{V}}(V)$. Define $u_{1}:=(1-\chi) u$ and $u_{2}:=\chi u$. Then it holds

(i) $\operatorname{supp}\left(u_{1}\right) \cap B_{\alpha_{1} h_{V}}(V)=\emptyset$,

(ii) $\operatorname{supp}\left(u_{2}\right)$ is contained in a single chart parametrizing the surface $\Gamma$,

(iii) $\left\|u_{2}\right\|_{\widetilde{H}_{h}^{\theta}\left(B_{\alpha_{2} h_{V}}(V) \cap \Gamma\right)} \lesssim\|u\|_{H_{h}^{\theta}\left(\omega_{V}\right)}$. 
While (ii) and (iii) follow directly from the construction and the properties of $\chi$, property (iii) is seen as follows: Note that the map $u \mapsto u_{2}$ is bounded as $L^{2}\left(\omega_{V}\right) \rightarrow L^{2}\left(B_{\alpha_{2} h_{V}}(V) \cap \Gamma\right)$ and $H_{h}^{1}\left(\omega_{V}\right) \rightarrow \widetilde{H}_{h}^{1}\left(B_{\alpha_{2} h_{V}}(V) \cap \Gamma\right)$. To see the second boundedness, note that

$$
\|\nabla(\chi u)\|_{L^{2}\left(B_{\alpha_{2} h_{V}}(V) \cap \Gamma\right)} \leq\|\chi\|_{L^{\infty}\left(\omega_{V}\right)}\|\nabla u\|_{L^{2}\left(B_{\alpha_{2} h_{V}}(V) \cap \Gamma\right)}+\|\nabla \chi\|_{L^{\infty}\left(\omega_{V}\right)}\|u\|_{L^{2}\left(B_{\alpha_{2} h_{V}}(V) \cap \Gamma\right)} \lesssim\|u\|_{H_{h}^{1}\left(\omega_{V}\right)}
$$

since $\|\nabla \chi\|_{L^{\infty}\left(\omega_{V}\right)} \sim \operatorname{dist}\left(B_{\alpha_{1} h_{V}}(V), \partial B_{\alpha_{2} h_{V}}(V)\right)^{-1} \sim h_{V}^{-1}$, and that $u_{2} \in \widetilde{H}_{h}^{1}\left(B_{\alpha_{2} h_{V}}(V) \cap \Gamma\right)$. An interpolation argument and Lemma 4.1 then show (iii). From (ii) it follows that

$$
\left\|d_{V}^{-\theta} u\right\|_{L^{2}\left(\omega_{V}\right)} \leq\left\|d_{V}^{-\theta} u_{1}\right\|_{L^{2}\left(\omega_{V}\right)}+\left\|d_{V}^{-\theta} u_{2}\right\|_{L^{2}\left(\omega_{V}\right)} \lesssim h_{V}^{-\theta}\|u\|_{L^{2}\left(\omega_{V}\right)}+\left\|d_{V}^{-\theta} u_{2}\right\|_{L^{2}\left(\omega_{V}\right)},
$$

and hence it suffices to show (4.14) for $u_{2}$.

Let $F_{\Gamma}: \mathbb{R}^{2} \rightarrow \mathbb{R}^{3}$ denote the chart parametrizing a neighborhood of $\operatorname{supp} u_{2}$. We use the set $Z$ and the parameter $\beta$ from Lemma 4.8. Since the map $F_{\Gamma}$ is bi-Lipschitz, we can assume (after possibly further reducing $\beta$ ):

$$
\bigcup_{z \in F_{\Gamma}^{-1}(Z)} \overline{B_{\beta d_{F_{\Gamma}^{-1}(V)}(z)}(z)} \subseteq F_{\Gamma}^{-1}(K)
$$

Now we apply Lemma 4.6 with $\omega=F_{\Gamma}^{-1}\left(B_{c_{1} h_{V}}(V) \cap \omega_{V}\right)$ and $\widetilde{\omega}=F_{\Gamma}^{-1}\left(B_{\alpha_{2} h_{V}}(V) \cap \omega_{V}\right)$ to obtain a function $\widehat{w}$. Then we take the push forward to (a subset of) $\omega_{V}$ via $F_{\Gamma}$ and extend it by zero to $\omega_{V}$. This function satisfies

$$
\int_{0}^{\infty} t^{-2 \theta}\left(\left\|u_{2}-w(t)\right\|_{L^{2}\left(\omega_{V}\right)}^{2}+t^{2}\|w(t)\|_{H_{h}^{1}\left(\omega_{V}\right)}^{2}\right) \frac{d t}{t} \lesssim\left\|u_{2}\right\|_{\widetilde{H}_{h}^{\theta}\left(B_{\alpha_{2} h_{V}}(V) \cap \omega_{V}\right)}^{2}
$$

and, using $\omega^{\prime}=F_{K}^{-1}\left(A^{2,1}(t) \cap Z\right)$ in Lemma 4.6 and (4.15) we get (by again taking the push forward and possibly further decreasing $\beta$ )

$$
\|w(t)\|_{L^{2}\left(A^{2,1}(t) \cap Z\right)} \lesssim\left\|u_{2}\right\|_{L^{2}\left(A^{3,1 / 2}(t) \cap K\right)} .
$$

Next, note that for a function $v$ we have

$$
\begin{aligned}
\|v\|_{L^{2}\left(\omega_{V}\right)}^{2} & \lesssim \int_{\omega_{V}}|v(x)|^{2} \log (2) d x \lesssim \int_{\omega_{V}}|v(x)|^{2} \int_{d_{V}(x) / 2}^{d_{V}(x)} \frac{d t}{t} d x \\
& =\int_{0}^{\infty} \int_{\omega_{V}}|v(x)|^{2} \chi_{A^{2,1}(t)}(x) d x \frac{d t}{t}=\int_{0}^{\infty}\|v\|_{L^{2}\left(A^{2,1}(t) \cap \omega_{V}\right)}^{2} \frac{d t}{t} .
\end{aligned}
$$

Hence, applying (4.12) to $w$, we estimate

$$
\begin{aligned}
\| d_{V}^{-\theta} & u_{2}\left\|_{L^{2}\left(\omega_{V}\right)}^{2} \lesssim \int_{t=0}^{\infty}\right\| d_{V}^{-\theta} u_{2} \|_{L^{2}\left(A^{2,1}(t) \cap \omega_{V}\right)}^{2} \frac{d t}{t} \\
& \lesssim \int_{t=0}^{\infty}\left\|d_{V}^{-\theta}\left(u_{2}-w(t)\right)\right\|_{L^{2}\left(A^{2,1}(t) \cap \omega_{V}\right)}^{2} \frac{d t}{t}+\int_{t=0}^{\infty} t^{2-2 \theta}\left\|d_{V}^{-1} w(t)\right\|_{L^{2}\left(A^{2,1}(t) \cap \omega_{V}\right)}^{2} \frac{d t}{t} \\
& \lesssim \int_{t=0}^{\infty} t^{-2 \theta}\left(\left\|\left(u_{2}-w(t)\right)\right\|_{L^{2}\left(A^{2,1}(t) \cap \omega_{V}\right)}^{2}+t^{2}\left\|d_{V}^{-1} w(t)\right\|_{L^{2}\left(A^{2,1}(t) \cap K \cap Z\right)}^{2}+t^{2}\|w(t)\|_{H_{h}^{1}\left(A^{2,1}(t) \cap \omega_{V}\right)}^{2}\right) \frac{d t}{t} \\
& \leq \int_{t=0}^{\infty} t^{-2 \theta}\left(\left\|u_{2}-w(t)\right\|_{L^{2}\left(\omega_{V}\right)}^{2}+t^{2}\|w(t)\|_{H_{h}^{1}\left(\omega_{V}\right)}^{2}\right) \frac{d t}{t}+\int_{t=0}^{\infty} t^{2-2 \theta}\left\|d_{V}^{-1} w(t)\right\|_{L^{2}\left(A^{2,1}(t) \cap K \cap Z\right)}^{2} \frac{d t}{t},
\end{aligned}
$$

where we used that $d_{V}^{-1} \sim t^{-1}$ on $A^{2,1}(t)$. Using additionally (4.17), we obtain

$$
\int_{t=0}^{\infty} t^{2-2 \theta}\left\|d_{V}^{-1} w(t)\right\|_{L^{2}\left(A^{2,1}(t) \cap Z\right)}^{2} \frac{d t}{t} \lesssim \int_{0}^{\infty} t^{2-2 \theta}\left\|d_{V}^{-1} u_{2}\right\|_{L^{2}\left(A^{3,1 / 2}(t) \cap K\right)}^{2} \frac{d t}{t} \lesssim\left\|d_{V}^{-\theta} u_{2}\right\|_{L^{2}(K)}^{2},
$$


where the last estimate can be seen using polar coordinates. Using also (4.16) and point (iii) above shows

$$
\left\|d_{V}^{-\theta} u_{2}\right\|_{L^{2}\left(\omega_{V}\right)} \lesssim\left\|d_{V}^{-\theta} u\right\|_{L^{2}(K)}+\|u\|_{H_{h}^{\theta}\left(\omega_{V}\right)} .
$$

\section{Averaging operators}

A key tool in the definition of the localization procedure are suitable averaging operators. Given a closed set $M$ (which will be a corner or an edge of $\widehat{K}$ ) the basic averaging operator is defined in Lemma 5.2 by locally averaging on a length scale of size $\mathcal{O}(\operatorname{dist}(\cdot, M))$. In this way function values are reproduced on $M$. This basic operator is modified in several steps in order to yield functions that vanish on parts of $\widehat{K}$. We study the stability of these operators in weighted and unweighted norms.

\subsection{Preliminaries}

The proof of stability of the local averaging operators relies on the following covering lemma.

Lemma 5.1 (covering lemma). Let $M \neq \emptyset$ be a closed subset of $\mathbb{R}^{d}$. Fix $\beta \in(0,1)$ and $c \in(0,1)$ such that

$$
(1+\beta) c<1 .
$$

For each $x \in \mathbb{R}^{d} \backslash M$ denote by $B_{x}:=\bar{B}_{c d_{M}(x)}(x)$ and $\widehat{B}_{x}:=\bar{B}_{(1+\beta) c d_{M}(x)}(x)$ closed balls centered at $x$ with radii $c d_{M}(x),(1+\beta) c d_{M}(x)$, respectively. Let $\omega \subset \mathbb{R}^{d} \backslash M$ be open. Then, there exist $\left(x_{i}\right)_{i \in \mathbb{N}} \subset \omega$ and $a$ constant $N \in \mathbb{N}$ depending solely on the spatial dimension $d$ such that:

(i) (covering property) $\omega \subset \cup_{i} B_{x_{i}}$;

(ii) (finite overlap) $\sup _{x \in \mathbb{R}^{d}} \operatorname{card}\left\{i: x \in \widehat{B}_{x_{i}}\right\} \leq N$.

Proof. The proof is very similar to that of [MW12, Lemma A.1]. We have to address the technical issue that the radii of the balls are unbounded so that it is not a priori clear that the classical Besicovitch covering theory is applicable. Fix $c_{0}>0$ such that $(1+\beta) c<1-c_{0}$ and define: $q:=\max \left\{1+c_{0}, 1 /\left(1-c_{0}\right)\right\}>1$. Define, for each $i \in \mathbb{Z}$, the bounded sets $\omega_{i}:=\left\{x \in \omega: q^{i} \leq d_{M}(x) \leq q^{i+1}\right\}$. For each of these sets, we can find a cover by balls of the above type. The choice of $q>1$ is such that balls with centers in $\omega_{i}$ have non-trivial intersection with $\omega_{j}$ only for $j \in\{i-1, i, i+1\}$. Hence, the overlap properties can be ensured.

The basic lemma for defining the local averaging operators is the following.

Lemma 5.2 (basic averaging operator). Let $M \neq \emptyset$ be a closed subset of $\mathbb{R}^{d}$. Fix $\alpha \in(0,1 / 3)$ and let $\rho \in C_{0}^{\infty}\left(\mathbb{R}^{d}\right)$ satisfy $\operatorname{supp} \rho \subset B_{\alpha}(0)$ and $\int_{\mathbb{R}^{d}} \rho(y) d y=1$. For $t>0$, set $\rho_{t}(y):=t^{-d} \rho(y / t)$. Define the averaging operator $\mathcal{A}_{\rho}^{M}: L_{\text {loc }}^{1}\left(\mathbb{R}^{d}\right) \rightarrow C^{\infty}\left(\mathbb{R}^{d} \backslash M\right)$ by

$$
\left(\mathcal{A}_{\rho}^{M} u\right)(x):=\int_{\mathbb{R}^{d}} u(y) \rho_{d_{M}(x)}(x-y) d y
$$

and for a set $\omega \subset \mathbb{R}^{d} \backslash M$ the domain of influence

$$
\omega_{\rho}^{M}:=\cup_{y \in \omega} \operatorname{supp} \rho_{d_{M}(y)}(y-\cdot) .
$$

Then, the following holds:

(i) $\mathcal{A}_{\rho}^{M} 1=1$.

(ii) If $\omega \subset \mathbb{R}^{d} \backslash M$ is open, then the conditions $\left.d_{M}\right|_{\omega_{\rho}^{M}} \in \mathcal{P}_{1}$ and $\left.u\right|_{\omega_{\rho}^{M}} \in \mathcal{P}_{p}$ imply $\left.\left(\mathcal{A}_{\rho}^{M} u\right)\right|_{\omega} \in \mathcal{P}_{p}$.

Let a function $\varphi \in L^{\infty}\left(\mathbb{R}^{d}\right)$ be given that satisfies, for some $c \in(0,1), C>0$, the conditions

$$
C^{-1} \varphi(y) \leq \varphi(x) \leq C \varphi(y) \quad \forall y \in B_{c d_{M}(x)}(x) \quad \forall x \in \mathbb{R}^{d} \backslash M .
$$

Then for any open $\omega \subset \mathbb{R}^{d} \backslash M$, there holds:

(iii) $\left\|\varphi \mathcal{A}^{M} u\right\|_{L^{2}(\omega)} \lesssim\|\varphi u\|_{L^{2}\left(\omega_{\rho}^{M}\right)}$ provided the right-hand side is finite. 
(iv) $\left\|\varphi \nabla \mathcal{A}^{M} u\right\|_{L^{2}(\omega)} \lesssim\|\varphi \nabla u\|_{L^{2}\left(\omega_{\rho}^{M}\right)}$ provided the right-hand side is finite.

(v) $\mathcal{A}_{\rho}^{M}: L^{2}\left(\omega_{\rho}^{M}\right) \rightarrow L^{2}(\omega)$ and $\mathcal{A}_{\rho}^{M}: H^{1}\left(\omega_{\rho}^{M}\right) \rightarrow H^{1}(\omega)$ is bounded.

(vi) If $u$ is continuous at a point $z \in M$, then $\mathcal{A}_{\rho}^{M} u$ is continuous at $z$ and $\left(\mathcal{A}_{\rho}^{M} u\right)(z)=u(z)$.

(vii) If $u \in H^{\theta}\left(\mathbb{R}^{d} \backslash M\right)$, then $\left\|d_{M}^{-\theta}\left(u-\mathcal{A}_{\rho}^{M} u\right)\right\|_{L^{2}\left(\mathbb{R}^{d} \backslash M\right)} \lesssim\|u\|_{H^{\theta}\left(\mathbb{R}^{d} \backslash M\right)}$.

(viii) $\left\|\nabla \mathcal{A}_{\rho}^{M} u\right\|_{L^{2}(\omega)} \lesssim\left\|d_{M}^{-1} u\right\|_{L^{2}\left(\omega_{\rho}^{M}\right)}$ provided the right-hand side is finite.

(ix) $\left\|d_{M} \nabla \mathcal{A}_{\rho}^{M} u\right\|_{L^{2}(\omega)} \lesssim\|u\|_{L^{2}\left(\omega_{\rho}^{M}\right)}$.

(x) Assume that $\left.d_{M}\right|_{\omega_{\rho}^{M}} \in \mathcal{P}_{1}$, then $\left\|d_{M} \nabla^{2} \mathcal{A}_{\rho}^{M} u\right\|_{L^{2}(\omega)} \lesssim\|\nabla u\|_{L^{2}\left(\omega_{\rho}^{M}\right)}$.

(xi) Let $K \subset \mathbb{R}^{d}$ be compact with $\operatorname{dist}(K, M)>0$. Then $\left\|\mathcal{A}_{\rho}^{M} u\right\|_{W^{j, \infty}(K)} \leq C_{K, \rho, d, M, j}\|u\|_{L^{2}\left(K_{\rho}^{M}\right)}$ for all $j \in \mathbb{N} \cup\{0\}$.

All hidden constants depend solely on $d, \rho, M$, and $\theta$.

Proof. We start with some preparatory results.

- Elementary considerations show that $d_{M}: \mathbb{R}^{d} \rightarrow \mathbb{R}$ is Lipschitz continuous with Lipschitz constant $L=1$.

- Let $\varphi$ satisfy (5.2). Then for any $\widehat{c} \in(0,1)$ there exists $\widehat{C}>0$ such that

$$
\widehat{C}^{-1} \varphi(y) \leq \varphi(x) \leq \widehat{C} \varphi(y) \quad \forall y \in B_{\widehat{c} d_{M}(x)}(x) \quad \forall x \in \mathbb{R}^{d} \backslash M .
$$

To see this, it suffices to consider the case $1>\widehat{c}>c$. For fixed $x \in \mathbb{R}^{d} \backslash M$ and $y \in B_{\widehat{c} d_{M}(x)}(x)$, consider a sequence of $L=\lfloor\widehat{c} /(c(1-\widehat{c}))\rfloor+1$ points $x=x_{0}, \ldots, x_{L}=y$ on the line connecting $x=x_{0}$ with $y=x_{L}$ such that $x_{i} \in B_{c d_{M}\left(x_{i-1}\right)}\left(x_{i-1}\right), i=1, \ldots, L$. For each pair $\left(x_{i-1}, x_{i}\right)$, the assumption (5.2) is applicable. Hence, the claim (5.3) follows with $\widehat{C}:=C^{L}$.

- A change of variables yields

$$
\left(\mathcal{A}_{\rho}^{M} u\right)(x)=\int_{B_{\alpha}(0)} u\left(x-d_{M}(x) y\right) \rho(y) d y .
$$

Proof of (ii) and (ii): This follows by a direct calculation.

Proof of (iii): First, we provide a locally finite cover of $\omega$. Recall $\alpha \in(0,1 / 3)$. Fix $c \in(0,1 / 2)$ such that $\alpha<c /(1+c)$. Then fix $\beta \in(0,1)$ such that $\alpha(1+c) / c<\beta$ and observe

$$
c<c+(1+c) \alpha<c(1+\beta)<1 .
$$

According to Lemma 5.1, there are points $x_{i} \in \omega, i \in \mathbb{N}$, such that the balls $B_{x_{i}}:=\bar{B}_{c d_{M}\left(x_{i}\right)}\left(x_{i}\right)$ and the stretched balls $\widehat{B}_{x_{i}}:=\bar{B}_{(1+\beta) c d_{M}\left(x_{i}\right)}\left(x_{i}\right)$ fulfill

$$
\begin{array}{r}
\omega \subset \cup_{i} B_{x_{i}}, \\
\sup _{x \in \mathbb{R}^{d}} \operatorname{card}\left\{i: x \in \widehat{B}_{x_{i}}\right\} \leq N \in \mathbb{N} .
\end{array}
$$

Setting $\widetilde{B}_{x_{i}}:=\bar{B}_{(c+(1+c) \alpha) d_{M}\left(x_{i}\right)}\left(x_{i}\right)$ and taking into account (5.5)), we also conclude

$$
\sup _{x \in \mathbb{R}^{d}} \operatorname{card}\left\{i: x \in \widetilde{B}_{x_{i}}\right\} \leq N
$$

Furthermore, it follows from the Lipschitz continuity of $d_{M}$ with constant $L=1$ that $y \in B_{x_{i}} \cap \omega$ implies $\operatorname{supp} \rho_{d_{M}(y)}(y-\cdot) \subset \widetilde{B}_{x_{i}}$, which yields

$$
\left(B_{x_{i}} \cap \omega\right)_{\rho}^{M} \subset \widetilde{B}_{x_{i}}
$$

and consequently

$$
\sup _{x \in \mathbb{R}^{d}} \operatorname{card}\left\{i: x \in\left(B_{x_{i}} \cap \omega\right)_{\rho}^{M}\right\} \leq N .
$$


For any $z \in \omega$, we have the basic estimate

$$
\left|\mathcal{A}_{\rho}^{M} u(z)\right| \lesssim d_{M}(z)^{-d}\|u\|_{L^{1}\left(\operatorname{supp} \rho_{d_{M}(z)}(z-\cdot)\right)}
$$

For $i \in \mathbb{N}$ we conclude, using Hölder's inequality and (5.3),

$$
\left\|\varphi \mathcal{A}_{\rho}^{M} u\right\|_{L^{2}\left(B_{x_{i}} \cap \omega\right)} \lesssim\|\varphi u\|_{L^{2}\left(\left(B_{x_{i}} \cap \omega\right)_{\rho}^{M}\right)} .
$$

The covering property (5.6a) and properties (5.7) then yield the result.

Proof of (iv): By Rademacher's Theorem [Fed69, Thm. 3.1.6], $d_{M}$ is differentiable almost everywhere with $\left|\nabla d_{M}(x)\right| \leq 1$. Using (5.4), we then write

$$
\nabla \mathcal{A}_{\rho}^{M} u(x)=\int_{B_{\alpha}(0)}\left(I-\nabla d_{M}(x) y^{T}\right) \nabla u\left(x-d_{M}(x) y\right) \rho(y) d y
$$

Now we can argue exactly as in the proof of (iii).

Proof of (豆): follows from (iii) and (iv) with $\varphi \equiv 1$.

Proof of (vi): This follows from (5.4) and the observation $\lim _{x^{\prime} \rightarrow x} d_{M}\left(x^{\prime}\right)=0$ for $x \in M$.

Proof of (vii): We start with the case $\theta=1$. Estimate (5.9), (i), and a Poincaré inequality show $\left\|\mathcal{A}_{\rho}^{M} u-u\right\|_{L^{2}\left(B_{x_{i}}\right)} \lesssim d_{M}\left(x_{i}\right)\|\nabla u\|_{L^{2}\left(\widetilde{B}_{x_{i}}\right)}$. Dividing by $d_{M}\left(x_{i}\right)$ and using that $d_{M}\left(x_{i}\right) \sim d_{M}$ on $B_{x_{i}}$ yields

$$
\left\|d_{M}^{-1}\left(\mathcal{A}_{\rho}^{M} u-u\right)\right\|_{L^{2}\left(B_{x_{i}}\right)} \lesssim\|\nabla u\|_{L^{2}\left(\widetilde{B}_{x_{i}}\right)} .
$$

Now we can argue as in the proof of (iii). The case of general $\theta \in(0,1)$ follows by interpolation.

Proof of (viii) and (ix): We calculate

$$
\begin{aligned}
\partial_{x_{j}}\left[\rho\left(\frac{x-y}{d_{M}(x)}\right) d_{M}(x)^{-d}\right]= & \rho\left(\frac{x-y}{d_{M}(x)}\right)(-d) d_{M}(x)^{-d-1} \partial_{x_{j}} d_{m}(x) \\
& +d_{M}(x)^{-d}\left(-(x-y) \cdot \nabla \rho\left(\frac{x-y}{d_{M}(x)}\right) \frac{\partial_{x_{j}} d_{M}(x)}{d_{M}(x)^{2}}+\partial_{x_{j}} \rho\left(\frac{x-y}{d_{M}(x)}\right) \frac{1}{d_{M}(x)}\right)
\end{aligned}
$$

and conclude for any $y \in B_{\alpha d_{M}(x)}(x)$ that

$$
\left|\nabla_{x} \rho_{d_{M}(x)}(x-y)\right| \lesssim d_{M}(x)^{-d-1} .
$$

Hence, we have the basic estimate

$$
\left|\nabla \mathcal{A}_{\rho}^{M} u(x)\right| \lesssim d_{M}(x)^{-d-1}\|u\|_{L^{1}\left(\operatorname{supp} \rho_{d_{M}(x)}(x-\cdot)\right)}
$$

and can proceed as in the proof of (iii).

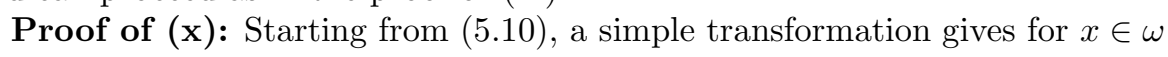

$$
\nabla \mathcal{A}_{\rho}^{M} u(x)=\int_{\omega_{\rho}^{M}}\left(I-\nabla d_{M}(x) d_{M}^{-1}(x)(x-y)^{T}\right) \nabla u(y) \rho_{d_{M}(x)}(x-y) d y .
$$

The assumption $d_{M} \in \mathcal{P}_{1}$ implies that $\nabla d_{M}$ is constant. Differentiating with respect to $x$, we can continue as in the proof of (ix). In particular, the additional term $(x-y) d_{M}^{-1}(x)$ and its $x$-derivative behave like $\mathcal{O}(1)$ and $\mathcal{O}\left(d_{M}^{-1}\right)$, leading to a differentiated version of (5.11).

Proof of (xi): This follows immediately from the basic estimates (5.8) and (5.11) for $j=0$, respectively $j=1$. The case $j>1$ can be shown analogously. 


\subsection{Averaging operators for the vertex part}

We first construct the averaging operators for the vertex contributions. Since it already showcases most of the difficulties encountered also for the other contributions, we present the construction in more detail.

\subsubsection{Averaging operators on the reference triangle for the vertex part}

We start with a simple averaging operator on the reference triangle, which will be the basis for the construction of further operators with additional properties.

Lemma 5.3. Let $\widehat{V}$ be a vertex of $\widehat{T}$. There exists a linear operator $\mathcal{A}^{\widehat{V}}: L_{\text {loc }}^{1}(\widehat{T}) \rightarrow C^{\infty}(\widehat{T})$ with the following properties:

(i) If $u$ is continuous at $\widehat{V}$, then $\left(\mathcal{A}^{\widehat{V}} u\right)(\widehat{V})=u(\widehat{V})$.

(ii) $\mathcal{A}^{\widehat{V}} 1 \equiv 1$. Furthermore, $u \in \mathcal{P}_{p}$ implies $\mathcal{A}^{\widehat{V}} u \in \mathcal{P}_{p}$.

(iii) $\mathcal{A}^{\widehat{V}}: H^{\theta}(\widehat{T}) \rightarrow H^{\theta}(\widehat{T})$ is bounded and linear for $\theta \in[0,1]$.

(iv) For fixed $\gamma \in \mathbb{R},\left\|d_{\widehat{V}}^{\gamma} \mathcal{A}^{\widehat{V}} u\right\|_{L^{2}(\widehat{T})} \leq C_{\gamma}\left\|d_{\widehat{V}}^{\gamma} u\right\|_{L^{2}(\widehat{T})}$ provided the right-hand side is finite.

(v) For all $\theta \in[0,1]$ there exists $C>0$ such that for all $u \in H^{\theta}(\widehat{T})$ :

$$
\left\|d_{\widehat{V}}^{-\theta}\left(u-\mathcal{A}^{\widehat{V}} u\right)\right\|_{L^{2}(\widehat{T})} \leq C\|u\|_{H^{\theta}(\widehat{T})} .
$$

(vi) For every $\varepsilon>0$ we have $\mathcal{A}^{\widehat{V}} u \in C^{\infty}\left(T_{\varepsilon}\right)$, where $T_{\varepsilon}:=\left\{(\xi, \eta) \in \widehat{\widehat{T}} \mid d_{\widehat{V}}(\xi, \eta) \geq \varepsilon\right\}$. Moreover, for every $j \in \mathbb{N}_{0}$ and $\varepsilon>0$, there exists $C_{j, \varepsilon}>0$ such that

$$
\left\|\mathcal{A}^{\widehat{V}} u\right\|_{W^{j, \infty}\left(T_{\varepsilon}\right)} \leq C_{j, \varepsilon}\|u\|_{L^{2}(\widehat{T})} .
$$

(vii) Let $\widehat{e}$ denote an edge of $\widehat{T}$ with $\widehat{V} \in \overline{\hat{e}}$, then

$$
\left\|d_{\widehat{V}}^{-1 / 2} \mathcal{A}^{\widehat{V}} u\right\|_{L^{2}(\widehat{e})} \leq C\left\|d_{\widehat{V}}^{-1} u\right\|_{L^{2}(\widehat{T})} .
$$

Proof. To fix ideas, we assume that $\widehat{V}=(0,0)$. We will apply Lemma 5.2, where we assume additionally that $\operatorname{supp} \rho \subset B_{\alpha}(0) \cap \widehat{T}$ for some $\alpha \in(0,1 / 3)$. We choose $\beta<1$ with $\beta(\sqrt{2}+\alpha)<1$ and the set $M=\{0\} \times \mathbb{R}$. Define $\widehat{u}(x):=u(\beta x)$ for $x \in \widehat{T}$, and extend $\widehat{u}$ to $\mathbb{R}^{d}$ using the Stein extension [Ste70, Sec. VI], which is simultaneously bounded in $L^{2}$ and $H^{1}$. Note that $\widehat{u}(\widehat{V})=u(\widehat{V})$ if $u$ is continuous at $\widehat{V}$. Now we define $\mathcal{A}^{\widehat{V}} u:=\mathcal{A}_{\rho}^{M} \widehat{u}$ with the aid of Lemma [5.2, Note that due to the choice of $\beta$ and the support of $\rho,\left.\left(\mathcal{A}^{\widehat{V}} u\right)\right|_{\widehat{T}}$ depends solely on $\left.u\right|_{\widehat{T}}$.

Proof of (ii): This follows from Lemma 5.2, (vil) and the fact that $\widehat{u}(\widehat{V})=u(\widehat{V})$.

Proof of (iii): The choice of $M$ implies $\left.d_{M}\right|_{\{x>0\}} \in \mathcal{P}_{1}$. Furthermore, $u \in \mathcal{P}_{p}(\widehat{T})$ implies $\widehat{u} \in \mathcal{P}_{p}\left(\beta^{-1} \widehat{T}\right)$, and we conclude with Lemma 5.2, (iii) that $\mathcal{A}^{\widehat{V}} u \in \mathcal{P}_{p}(\widehat{T})$. Together with Lemma 5.2, (ii), this shows (iii).

Proof of (iii): For $\theta \in\{0,1\}$, note that due to Lemma [5.2, (V) and $\widehat{T}_{\rho}^{M} \subset \beta^{-1} \widehat{T}$,

$$
\left\|\mathcal{A}^{\widehat{V}} u\right\|_{H^{\theta}(\widehat{T})} \lesssim\|\widehat{u}\|_{H^{\theta}\left(\beta^{-1} \widehat{T}\right)} \lesssim\|u\|_{H^{\theta}(\widehat{T})} .
$$

An interpolation argument finishes the proof.

Proof of (iv): We note that due to Lipschitz continuity of $d_{M}$, the function $\varphi:=d_{M}^{\gamma}$ fulfills (5.2). Hence, the estimate follows from Lemma 5.2. (iii).

Proof of ( (v) : We calculate, using $d_{\widehat{V}} \sim d_{M}$ on $\widehat{T}$,

$$
\left\|d_{\widehat{V}}^{-\theta}\left(u-\mathcal{A}^{\widehat{V}} u\right)\right\|_{L^{2}(\widehat{T})} \lesssim\left\|d_{M}^{-\theta}\left(u-\mathcal{A}^{\widehat{V}} u\right)\right\|_{L^{2}(\widehat{T})} \leq\left\|d_{M}^{-\theta}\left(\widehat{u}-\mathcal{A}_{\rho}^{M} \widehat{u}\right)\right\|_{L^{2}\left(\mathbb{R}^{d} \backslash M\right)}+\left\|d_{M}^{-\theta}(u-\widehat{u})\right\|_{L^{2}(\widehat{T})} .
$$

Next,

$$
\left\|d_{M}^{-\theta}\left(\widehat{u}-\mathcal{A}_{\rho}^{M} \widehat{u}\right)\right\|_{L^{2}\left(\mathbb{R}^{d} \backslash M\right)} \lesssim\|\widehat{u}\|_{H^{\theta}\left(\mathbb{R}^{d} \backslash M\right)} \lesssim\|u\|_{H^{\theta}(\widehat{T})},
$$


where the first estimate follows from Lemma [5.2, (vii), and the last one is due to the boundedness of the Stein extension operator and the definition of $\widehat{u}$. It remains to consider $\left\|d_{M}^{-\theta}(u-\widehat{u})\right\|_{L^{2}(\widehat{T})}$. For $\theta=0$, this term can be bounded by $\|u\|_{L^{2}(\widehat{T})}$. For $\theta=1$, define the function $\widetilde{u}(x, y)=u(x, \beta y)$ and estimate

$$
\begin{aligned}
\int_{\widehat{T}} d_{M}^{-2} & (u(x, \beta y)-u(\beta x, \beta y))^{2} d x d y \leq \int_{0}^{1} \int_{y}^{1}\left(x^{-1} \int_{\beta x}^{x} \partial_{1} u(s, \beta y) d s\right)^{2} d x d y \\
& \leq \int_{0}^{1} \int_{y}^{1}\left(x^{-1} \int_{0}^{x}\left|\partial_{1} u(s, \beta y)\right| d s\right)^{2} d x d y \lesssim \int_{0}^{1} \int_{y}^{1}\left|\partial_{1} u(x, \beta y)\right|^{2} d x d y \\
& \lesssim \int_{\widehat{T}}\left|\partial_{1} u(x, y)\right|^{2} d x d y,
\end{aligned}
$$

where we used Hardy's inequality in the second step. Likewise,

$$
\begin{gathered}
\int_{\widehat{T}} d_{M}^{-2}(u(x, y)-u(x, \beta y))^{2} d x d y \leq \int_{0}^{1} \int_{0}^{x}\left(x^{-1} \int_{0}^{y}\left|\partial_{2} u(x, s)\right| d s\right)^{2} d y d x \\
\leq \int_{0}^{1} \int_{0}^{x}\left(y^{-1} \int_{0}^{y}\left|\partial_{2} u(x, s)\right| d s\right)^{2} d y d x \lesssim \int_{0}^{1} \int_{0}^{x}\left|\partial_{2} u(x, y)\right|^{2} d y d x .
\end{gathered}
$$

The triangle inequality then shows that $\left\|d_{M}^{-1}(u-\widehat{u})\right\|_{L^{2}(\widehat{T})} \lesssim\|u\|_{H^{1}(\widehat{T})}$. The case of $\theta \in(0,1)$ follows by interpolation.

Proof of (vi): This follows from Lemma 5.2, (xi).

Proof of (vii): This follows from Lemma 4.4 and Lemma [5.2, (viii), using that $d_{\widehat{V}} \sim d_{M}$ on $\widehat{T}$.

We now modify $\mathcal{A}^{\widehat{V}}$ to construct an operator $\mathcal{A}_{0}^{\widehat{V}}$ that produces functions that vanish on one edge of $\widehat{T}$ and have a convenient symmetry, making the extension to a patch-supported function easier:

Lemma 5.4. Let $\widehat{V}$ be a vertex of $\widehat{T}$. There exists a linear operator $\mathcal{A}_{0}^{\widehat{V}}: L_{\text {loc }}^{1}(\widehat{T}) \rightarrow C^{\infty}(\widehat{T})$ with the following properties:

(i) If $u$ is continuous at $\widehat{V}$, then $\left(\mathcal{A}_{0}^{\widehat{V}} u\right)(\widehat{V})=u(\widehat{V})$.

(ii) $u \in \mathcal{P}_{p}$ implies $\mathcal{A}_{0}^{\widehat{V}} u \in \mathcal{P}_{p}$ for all $p \geq 1$.

(iii) $\mathcal{A}_{0}^{\widehat{V}}: H^{\theta}(\widehat{T}) \rightarrow H^{\theta}(\widehat{T})$ is bounded and linear for $\theta \in[0,1]$.

(iv) For fixed $\gamma \in[-1,0]$, there holds $\left\|d_{\widehat{V}}^{\gamma} \mathcal{A}_{0}^{\widehat{V}} u\right\|_{L^{2}(\widehat{T})} \leq C_{\gamma}\left\|d_{\widehat{V}}^{\gamma} u\right\|_{L^{2}(\widehat{T})}$ provided the right-hand side is finite.

(v) For all $\theta \in[0,1]$ there exists $C>0$ such that for $u \in H^{\theta}(\widehat{T})$

$$
\left\|d_{\widehat{V}}^{-\theta}\left(u-\mathcal{A}_{0}^{\widehat{V}} u\right)\right\|_{L^{2}(\widehat{T})} \leq C\|u\|_{H^{\theta}(\widehat{T})}
$$

(vi) For every $\varepsilon>0$ we have $\mathcal{A}_{0}^{\widehat{V}} u \in C^{\infty}\left(T_{\varepsilon}\right)$, where $T_{\varepsilon}:=\left\{(\xi, \eta) \in \overline{\widehat{T}} \mid d_{\widehat{V}}(\xi, \eta) \geq \varepsilon\right\}$. Moreover, for every $j \in \mathbb{N}_{0}$ and $\varepsilon>0$, there exists $C_{j, \varepsilon}>0$ such that

$$
\left\|\mathcal{A}_{0}^{\widehat{V}} u\right\|_{W^{j, \infty}\left(T_{\varepsilon}\right)} \leq C_{j, \varepsilon}\|u\|_{L^{2}(\widehat{T})} .
$$

(vii) Let e denote an edge of $\widehat{T}$ with $\widehat{V} \in \bar{e}$. Then

$$
\left\|d_{\widehat{V}}^{-1 / 2} \mathcal{A}_{0}^{\widehat{V}} u\right\|_{L^{2}(e)} \leq C\left\|d_{\widehat{V}}^{-1} u\right\|_{L^{2}(\widehat{T})} .
$$

(viii) Let $\widehat{e}$ denote the edge opposite $\widehat{V}$. Then $\left.\left(\mathcal{A}_{0}^{\widehat{V}} u\right)\right|_{\widehat{e}}=0$.

(ix) Let $\widehat{V}=(1,0)$. Then $\mathcal{A}_{0}^{\widehat{V}} u(\xi, \eta)=\mathcal{A}_{0}^{\widehat{V}} u(1-\eta, 1-\xi)$ for all $(\xi, \eta) \in \widehat{T}$.

(x) Let $V \neq \widehat{V}$ be another vertex of $\widehat{T}$. Then

$$
\left\|d_{V}^{-1 / 2} \mathcal{A}_{0}^{\widehat{V}} u\right\|_{L^{2}(\widehat{T})} \lesssim\|u\|_{L^{2}(\widehat{T})} \quad{ }_{22} \quad\left\|d_{V}^{-1 / 2} \nabla \mathcal{A}_{0}^{\widehat{V}} u\right\|_{L^{2}(\widehat{T})} \lesssim\|u\|_{H^{1}(\widehat{T})} .
$$


Proof. As in the proof of Lemma 5.3, we assume $\widehat{V}=(0,0)$. The operator $\mathcal{A}_{0}^{\widehat{V}}$ is constructed by a sequence of modifications of $u_{1}:=\mathcal{A}^{\widehat{V}} u$, where $\mathcal{A}^{\widehat{V}}$ is the operator from Lemma 5.3. Set $u_{2}(\xi, \eta):=u_{1}(\xi, \eta)-$ $l_{1}(\xi, \eta) u_{1}(1,0)-l_{2}(\xi, \eta) u_{1}(1,1)$, where $l_{1}$ and $l_{2}$ are the affine hat functions associated with the vertices $(1,0)$ and $(1,1)$. It follows immediately with Lemma 5.3 , (vil) that

$$
\left\|u_{2}\right\|_{W^{1, \infty}\left(T_{1 / 2}\right)} \lesssim\|u\|_{L^{2}(\widehat{T})}
$$

Define $g(\xi, \eta)=u_{2}(1, \eta)(\xi-\eta) /(1-\eta)$ and set $u_{3}(\xi, \eta):=u_{2}(\xi, \eta)-g(\xi, \eta)$. It follows immediately that $u_{3}(\widehat{V})=u(\widehat{V})$ if $u$ is continuous at $\widehat{V}$ and that $\left.u_{3}\right|_{\widehat{e}}=0$ if $\widehat{e}$ is the edge opposite to $\widehat{V}$. Using the properties of $\mathcal{A}^{\widehat{V}}$, we note

$$
\left\|u_{3}\right\|_{H^{\theta}(\widehat{T})} \lesssim\left\|\mathcal{A}^{\widehat{V}} u\right\|_{H^{\theta}(\widehat{T})}+\left\|\mathcal{A}^{\widehat{V}} u\right\|_{L^{\infty}\left(T_{1 / 2}\right)}+\|g\|_{H^{\theta}(\widehat{T})} \lesssim\|u\|_{H^{\theta}(\widehat{T})}+\|g\|_{H^{\theta}(\widehat{T})} .
$$

Using (5.14) we conclude $\|g\|_{L^{2}(\widehat{T})} \lesssim\|u\|_{L^{2}(\widehat{T})}$, and since $u_{2}$ vanishes to first order in the vertices $(1,0)$ and $(1,1)$, we also conclude from (5.14) that $\|g\|_{H^{1}(\widehat{T})} \lesssim\|u\|_{L^{2}(\widehat{T})}$. An interpolation argument shows $\|g\|_{H^{\theta}(\widehat{T})} \lesssim\|u\|_{L^{2}(\widehat{T})}$ and hence $\left\|u_{3}\right\|_{H^{\theta}(\widehat{T})} \lesssim\|u\|_{H^{\theta}(\widehat{T})}$. The results obtained so far and a triangle inequality yield $\left\|u-u_{3}\right\|_{L^{2}(\widehat{T})} \lesssim\|u\|_{L^{2}(\widehat{T})}$. Next, note that every term of $h:=u_{3}-\mathcal{A}^{\widehat{V}} u$ vanishes at least on one edge containing $\widehat{V}$. Hence, by Lemma 4.4 the triangle inequality, and the results obtained so far, we conclude

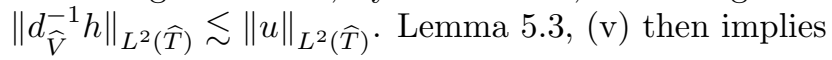

$$
\left\|d_{\widehat{V}}^{-1}\left(u-u_{3}\right)\right\|_{L^{2}(\widehat{T})} \leq\left\|d_{\widehat{V}}^{-1}\left(u-\mathcal{A}^{\widehat{V}} u\right)\right\|_{L^{2}(\widehat{T})}+\left\|d_{\widehat{V}}^{-1} h\right\|_{L^{2}(\widehat{T})} \lesssim\|u\|_{H^{1}(\widehat{T})} .
$$

An interpolation argument shows $\left\|d_{\widehat{V}}^{-\theta}\left(u-u_{3}\right)\right\|_{L^{2}(\widehat{T})} \lesssim\|u\|_{H^{\theta}(\widehat{T})}$. It is seen immediately that $u_{3}$ satisfies also the statement of Lemma 5.3, (vi). Finally, we will show that $u_{3}$ fulfills the statement of (vii). Due to Lemma 5.3, (vi) and (vii),

$$
\left\|d_{\widehat{V}}^{-1 / 2} u_{2}\right\|_{L^{2}(e)} \leq\left\|d_{\widehat{V}}^{-1 / 2} \mathcal{A}^{\widehat{V}} u\right\|_{L^{2}(e)}+\left(\left\|d_{\widehat{V}}^{-1 / 2} l_{1}\right\|_{L^{2}(e)}+\left\|d_{\widehat{V}}^{-1 / 2} l_{2}\right\|_{L^{2}(e)}\right)\left\|\mathcal{A}^{\widehat{V}} u\right\|_{L^{\infty}\left(T_{1 / 2}\right)} \lesssim\|u\|_{L^{2}(\widehat{T})},
$$

and furthermore

$$
\left\|d_{\widehat{V}}^{-1 / 2} g\right\|_{L^{2}(e)} \lesssim\left\|u_{2}\right\|_{L^{\infty}\left(T_{1 / 2}\right)} \lesssim\|u\|_{L^{2}(\widehat{T})} .
$$

A triangle inequality shows that $u_{3}$ fulfills the statement of Lemma 5.3, (vii). To show (iv) we note

$$
\begin{aligned}
\left\|d_{\widehat{V}}^{\gamma} u_{3}\right\|_{L^{2}(\widehat{T})} & \lesssim\left\|d_{\widehat{V}}^{\gamma} u_{2}\right\|_{L^{2}(\widehat{T})}+\left\|u_{2}\right\|_{L^{\infty}\left(T_{1 / 2}\right)} \lesssim\left\|d_{\widehat{V}}^{\gamma} u_{2}\right\|_{L^{2}(\widehat{T})}+\|u\|_{L^{2}(\widehat{T})} \\
& \lesssim\left\|d_{\widehat{V}}^{\gamma} \mathcal{A}^{\widehat{V}} u\right\|_{L^{2}(\widehat{T})}+\left\|\mathcal{A}^{\widehat{V}} u\right\|_{L^{\infty}\left(T_{1 / 2}\right)}+\|u\|_{L^{2}(\widehat{T})} \lesssim\left\|d_{\widehat{V}}^{\gamma} u\right\|_{L^{2}(\widehat{T})},
\end{aligned}
$$

where we used (5.14) for the second estimate and Lemma 5.3 for the last one. Finally, we set

$$
\mathcal{A}_{0}^{\widehat{V}} u(\xi, \eta):=u_{4}(\xi, \eta):=\frac{1}{2}\left(u_{3}(\xi, \eta)+u_{3}(\xi, \xi-\eta)\right) .
$$

If $\widehat{V}=(1,0)$ we take the average of $u_{3}(\xi, \eta)$ and $u_{3}(1-\eta, 1-\xi)$ instead.

Then, $u_{4}$ fulfills the same bounds as $u_{3}$ and satisfies (viii) and (ix). It is easy to see that $u_{4}(\widehat{V})=u(\widehat{V})$ and $u_{4}$ is continuous at $\widehat{V}$. Finally, inspection shows that if $u$ is a polynomial of degree $p \geq 1$, then $u_{4}$ is a polynomial of degree $p$. In order to prove (区) , we fix a smooth cut-off function $\phi$ that equals 1 in a neighborhood of $V$ and has support in a neighborhood of $V$ excluding the other vertices. Then,

$$
\left\|d_{V}^{-1 / 2} \mathcal{A}_{0}^{\widehat{V}} u\right\|_{L^{2}(\widehat{T})} \leq\left\|d_{V}^{-1 / 2} \phi \mathcal{A}_{0}^{\widehat{V}} u\right\|_{L^{2}(\widehat{T})}+\left\|d_{V}^{-1 / 2}(1-\phi) \mathcal{A}_{0}^{\widehat{V}} u\right\|_{L^{2}(\widehat{T})} .
$$


Due to the support properties of $\phi$, the second term on the right-hand side is bounded by $\left\|\mathcal{A}_{0}^{\widehat{V}} u\right\|_{L^{2}(\widehat{T})}$ and consequently by $\|u\|_{L^{2}(\widehat{T})}$. The first term on the right-hand side is bounded by $\left\|\phi \mathcal{A}_{0}^{\widehat{V}} u\right\|_{L^{\infty}(\widehat{T})}$ due to Lemma 4.5] and by the support properties of $\phi$ and (vi) finally by $\|u\|_{L^{2}(\widehat{T})}$. The estimate involving the gradient is shown analogously.

\subsubsection{Averaging operators on squares for the vertex parts}

The vertex averaging operator $\mathcal{A}_{\widehat{S}}$ (see Lemma 5.7 below) for the square is obtained from an averaging operator based on a triangle contained in $\widehat{S}$ and then extended to the full square by a Duffy transformation. Prior to defining $\mathcal{A}_{\widehat{S}}$, we therefore study the Duffy transformation in the following Lemma 5.5 .

Lemma 5.5. We introduce the Duffy transform

$$
T_{\mathcal{D}}: \quad \widehat{S} \rightarrow \widehat{T}, \quad\left(\begin{array}{l}
\xi \\
\eta
\end{array}\right) \mapsto\left(\begin{array}{c}
\eta(1-\xi)+\xi \\
\eta
\end{array}\right)
$$

and the corresponding linear operator as $\mathcal{D}: u \mapsto u \circ T_{\mathcal{D}}$. Then the following holds:

(i) $\mathcal{D}$ is bounded in the norms

$$
\begin{aligned}
\|\mathcal{D} u\|_{L^{2}(\widehat{S})} & \lesssim\left\|d_{(1,1)}^{-1 / 2} u\right\|_{L^{2}(\widehat{T})} \\
\|\mathcal{D} u\|_{H^{1}(\widehat{S})} & \lesssim\left\|d_{(1,1)}^{-1 / 2} u\right\|_{L^{2}(\widehat{T})}+\left\|d_{(1,1)}^{-1 / 2} \nabla u\right\|_{L^{2}(\widehat{T})}
\end{aligned}
$$

(ii) If $u \in \mathcal{P}_{p}(\widehat{T})$, then $\mathcal{D} u \in \mathcal{Q}_{p}(\widehat{S})$.

(iii) If $u$ vanishes on the edge $\widehat{e}_{2}:=\{(x, x), 0<x<1\}$, then $\mathcal{D} u$ vanishes on the edges $e_{2}:=(0,1) \times\{1\}$ and $e_{3}:=\{0\} \times(0,1)$,

(iv) The values on the edges $e_{0}:=(0,1) \times\{0\}$ and $e_{1}:=\{1\} \times(0,1)$ are preserved, i.e., $\mathcal{D} u(\xi, 0)=u(\xi, 0)$ and $\mathcal{D} u(1, \eta)=u(1, \eta)$.

(v) Close to the vertex $\widehat{V}:=(1,0)$, the Duffy transform is almost the identity in the sense that, provided that the right-hand side is finite,

$$
\left\|d_{\widehat{V}}^{-\theta}(u-\mathcal{D} u)\right\|_{L^{2}(\widehat{T})} \leq\|u\|_{H^{\theta}(\widehat{T})}+\left\|d_{(1,1)}^{-1 / 2} u\right\|_{L^{2}(\widehat{T})} .
$$

(vi) For $\widehat{V}:=(1,0)$ and $\gamma \in \mathbb{R}$ there holds

$$
\left\|d_{\widehat{V}}^{\gamma} \mathcal{D} u\right\|_{L^{2}(\widehat{S})} \leq\left\|d_{\widehat{V}}^{\gamma} u\right\|_{L^{2}(\widehat{T})}+\left\|d_{(1,1)}^{-1 / 2} u\right\|_{L^{2}(\widehat{T})} .
$$

Proof. Proof of (ii): The Jacobi matrix of $T_{\mathcal{D}}$ is $d T_{\mathcal{D}}=\left(\begin{array}{cc}1-\eta & 1-\xi \\ 0 & 1\end{array}\right)$, hence $\left|\operatorname{det} d T_{\mathcal{D}}\right|=1-\eta=1-y$. Transforming the integral we thus pick up the factor $(1-y)^{-1} \sim d_{(1,1)}^{-1}$.

Proof of (iii): Let $u=\sum_{i=0, j=0}^{p} \alpha_{i, j} x^{i} y^{j}$ with $i+j \leq p$. Inserting the definition of $\mathcal{D}$ gives: $\mathcal{D} u=$ $\sum_{i=0, j=0}^{p} \alpha_{i, j}(\eta(1-\xi)+\xi)^{i} \eta^{j}$. Expanding the powers and inspecting the highest polynomial degrees, we observe that the leading term has the form $\beta_{i, j} \eta^{i} \xi^{i} \eta^{j}=\beta_{i, j} \eta^{i+j} \xi^{i}$. Since $i+j \leq p$ we have $\mathcal{D} u \in \mathcal{Q}_{p}$.

Proof of (iii) and (iv): The claims follow by inspection.

Proof of (v): Fix a smooth cut-off function $\phi$ that equals 1 in a neighborhood of the vertex $(1,1)$ and whose support excludes neighborhoods of the other 2 vertices. Then

$$
\left\|d_{\widehat{V}}^{-\theta}(u-\mathcal{D} u)\right\|_{L^{2}(\widehat{T})} \leq\left\|d_{\widehat{V}}^{-\theta}(\phi u-\mathcal{D}(\phi u))\right\|_{L^{2}(\widehat{T})}+\left\|d_{\widehat{V}}^{-\theta}((1-\phi) u-\mathcal{D}((1-\phi) u))\right\|_{L^{2}(\widehat{T})} .
$$

The first term of the right-hand side can be estimated by

$$
\left\|d_{\widehat{V}}^{-\theta}(\phi u-\mathcal{D}(\phi u))\right\|_{L^{2}(\widehat{T})} \lesssim\|\phi u-\mathcal{D}(\phi u)\|_{L^{2}(\widehat{T})} \lesssim\left\|d_{(1,1)}^{-1 / 2} \phi u\right\|_{L^{2}(\widehat{T})} \lesssim\left\|d_{(1,1)}^{-1 / 2} u\right\|_{L^{2}(\widehat{T})},
$$


where we use the support properties of $\phi$ in the first step and (ii) in the second. For the second term on the right-hand side of (5.17) note that for $\theta=0$ we obtain with the properties of $\phi$

$$
\|(1-\phi) u-\mathcal{D}((1-\phi) u)\|_{L^{2}(\widehat{T})} \lesssim\left\|d_{(1,1)}^{-1 / 2}(1-\phi) u\right\|_{L^{2}(\widehat{T})} \lesssim\|u\|_{L^{2}(\widehat{T})}
$$

For $\theta=1$, note that $d_{\widehat{V}} \geq 1-\xi$ and calculate using Hardy's inequality

$$
\begin{aligned}
\int_{0}^{1} \int_{\eta}^{1} d_{\widehat{V}}^{-2}(v(\eta(1-\xi)+\xi, \eta)-v(\xi, \eta))^{2} d \xi d \eta & \leq \int_{0}^{1} \int_{\eta}^{1}\left((1-\xi)^{-1} \int_{\xi}^{1}\left|\partial_{1} v(s, \eta)\right| d s\right)^{2} d \xi d \eta \\
& \lesssim \int_{0}^{1} \int_{\eta}^{1}\left|\partial_{1} v(\xi, \eta)\right|^{2} d \xi d \eta .
\end{aligned}
$$

Applying this to $v=(1-\phi) u$ shows

$$
\left\|d_{\widehat{V}}^{-1}((1-\phi) u-\mathcal{D}((1-\phi) u))\right\|_{L^{2}(\widehat{T})} \lesssim|(1-\phi) u|_{H^{1}(\widehat{T})} \leq\|u\|_{H^{1}(\widehat{T})} .
$$

Interpolating this with (5.18) allows us to bound the second term on the right-hand side of (5.17).

Proof of (vi): Follows by transforming the integral and using $d_{\widehat{V}} \circ T_{\mathcal{D}} \sim d_{\widehat{V}}$.

When switching between polynomials on the reference square and reference triangle, the difference in the definition of $\mathcal{Q}_{p}$ and $\mathcal{P}_{p}$ leads to an increase of the degree by a factor of 2 . The main tool to correct the polynomial degrees on rectangles will be the Gauß-Lobatto interpolation operator. We collect its properties in the following lemma.

Lemma 5.6. (i) There holds for $\theta \in(0,1)$ and $p \in \mathbb{N}_{0}$, and $E$ a collection of edges of $\widehat{S}$ :

$$
\begin{aligned}
\left(\mathcal{Q}_{p},\|\cdot\|_{L^{2}(\widehat{S})}, \mathcal{Q}_{p},\|\cdot\|_{H^{1}(\widehat{S})}\right)_{\theta, 2} & =\left(\mathcal{Q}_{p},\|\cdot\|_{H^{\theta}(\widehat{S})}\right) \quad \text { (equivalent norms), } \\
\left(\widetilde{\mathcal{Q}}_{p}(\widehat{S}, E),\|\cdot\|_{L^{2}(\widehat{S})}, \widetilde{\mathcal{Q}}_{p}(\widehat{S}, E),\|\cdot\|_{H^{1}(\widehat{S})}\right)_{\theta, 2} & =\left(\widetilde{\mathcal{Q}}_{p}(\widehat{S}, E),\|\cdot\|_{\widetilde{H}^{\theta}(\widehat{S}, E)}\right) \quad \text { (equivalent norms), }
\end{aligned}
$$

where $\widetilde{\mathcal{Q}}_{p}(\widehat{S}, E) \subset \mathcal{Q}_{p}$ denotes the polynomials vanishing on $E$, and the $\widetilde{H}^{\theta}(\widehat{S}, E)$-norm is defined via interpolation of $L^{2}$ and $H^{1}(\widehat{S}) \cap\left\{u:\left.u\right|_{E}=0\right\}$. The constants in the norm equivalences do not depend on $p$.

(ii) Let $i_{p}: C(\overline{\widehat{S}}) \rightarrow \mathcal{Q}_{p}$ be the tensor-product Gauß-Lobatto interpolation operator. Then for every $\theta \in[0,1]$ there exists $C>0$ such that for all $p, q \in \mathbb{N}_{0}$ the following stability estimate holds for the operator $i_{p}$ :

$$
\left\|i_{p}\right\|_{\left(\mathcal{Q}_{p},\|\cdot\|_{H^{\theta}(\widehat{S})}\right) \leftarrow\left(\mathcal{Q}_{q},\|\cdot\|_{H^{\theta}(\widehat{S})}\right)} \leq C(1+q /(p+1))^{2-\theta}
$$

(iii) Let $\widehat{V}$ be a vertex of $\widehat{S}$ and set $d_{\widehat{V}}:=\operatorname{dist}(\cdot, \widehat{V})$. Then there exists a constant $C>0$, such that for all $p, q \in \mathbb{N}_{0}$, the following estimate holds:

$$
\left\|d_{\widehat{V}}^{-\theta}\left(u-i_{p} u\right)\right\|_{L^{2}(\widehat{S})} \leq C(1+q /(p+1))^{2(1-\theta)}(q / p)^{\theta}\|u\|_{H^{\theta}(\widehat{S})} \quad \forall u \in \mathcal{Q}_{q}(\widehat{S}) .
$$

Proof. Statement (ii) is the assertion of [BB94, Theorem 6]. For the treatment of boundary condition, see also Proposition 5 and its preceding remark. To show (iii), the key observation is that the 1D GaußLobatto interpolation operator $i_{p}^{G L}$ is stable in $H^{1}(-1,1)$ by [BM97, (13.27)] and also satisfies the stability estimate $\left\|i_{p}^{G L} u\right\|_{L^{2}(-1,1)} \leq C(1+q /(p+1))\|u\|_{L^{2}(-1,1)}$ for all $u \in \mathcal{P}_{q}$ by [BM97, Rem. 13.5]. Tensor product arguments then give for all $u \in \mathcal{Q}_{q}$ the estimates $\left\|i_{p} u\right\|_{L^{2}(\widehat{S})} \lesssim(1+q /(p+1))^{2}\|u\|_{L^{2}(\widehat{S})}$ and $\left\|i_{p} u\right\|_{H^{1}(\widehat{S})} \lesssim(1+q /(p+1))\|u\|_{H^{1}(\widehat{S})}$. Interpolation, which is possible due to (il), allows us to conclude the proof. To show (iii), note first that for $\theta=0$ the statement is equivalent to the $L^{2}$-stability of the GaußLobatto interpolation. For $\theta=1$, we note that by Lemma 4.4 it is sufficient to bound $\left\|d_{\widehat{V}}^{-1 / 2}\left(u-i_{p} u\right)\right\|_{L^{2}(\widehat{e})}$ 
for the two edges emanating from the vertex $\widehat{V}$. On such an edge $\widehat{e}$, the tensor product operator coincides with the 1D Gauß-Lobatto interpolation operator. We combine the approximation estimate [BM97, Theorem 13.4], an inverse estimate (see [Sch98, Theorem 3.91] for the $H^{1}-L^{2}$ case, the $H^{1}-H^{1 / 2}$ case follows by interpolation) and a trace estimate to get

$$
\left\|d_{\widehat{V}}^{-1 / 2}\left(u-i_{p} u\right)\right\|_{L^{2}(\widehat{e})} \lesssim\left\|\operatorname{dist}(\cdot, \partial \widehat{e})^{-1 / 2}\left(u-i_{p} u\right)\right\|_{L^{2}(\widehat{e})} \lesssim \frac{1}{p}\|u\|_{H^{1}(\widehat{e})} \lesssim \frac{q}{p}\|u\|_{H^{1 / 2}(\widehat{e})} \lesssim \frac{q}{p}\|u\|_{H^{1}(\widehat{S})} .
$$

The general statement then follows from interpolation and Proposition 3.1

Lemma 5.7. There exists an operator $\mathcal{A}_{\widehat{S}}: L_{\mathrm{loc}}^{1}(\widehat{S}) \rightarrow C^{\infty}(\widehat{T})$ such that

(i) $\mathcal{A}_{\widehat{S}}: H^{\theta}(\widehat{S}) \rightarrow H^{\theta}(\widehat{T})$ is linear and bounded for all $\theta \in[0,1]$.

(ii) $\mathcal{A}_{\widehat{S}}$ reproduces the value at $(1,0)$, i.e., $\left(\mathcal{A}_{\widehat{S}} u\right)(1,0)=u(1,0)$ if $u$ is continuous at $(1,0)$.

(iii) $\left\|d_{(1,0)}^{-\theta}\left(u-\mathcal{A}_{\widehat{S}} u\right)\right\|_{L^{2}(\widehat{T})} \lesssim\|u\|_{H^{\theta}(\widehat{S})}$.

(iv) $\mathcal{A}_{\widehat{S}} u$ vanishes on the edge of $\widehat{T}$ opposite to $(1,0)$.

(v) Let $e_{1}, e_{2}$ be the edges of $\widehat{S}$ with $(1,0) \in \overline{e_{1}} \cap \overline{e_{2}}$, and let $F_{e_{1}}^{e_{2}}$ be the affine map, mapping $e_{1}$ to $e_{2}$ with $F(1,0)=(1,0)$. Then the following holds for $\hat{u}:=\mathcal{A}_{\widehat{S}} u$ :

$$
\hat{u}(z)=\hat{u} \circ F_{e_{1}}^{e_{2}}(z) \quad \forall z \in e_{1} .
$$

(vi) If $u \in \mathcal{Q}_{p}(\widehat{S})$, then $\mathcal{A}_{\widehat{S}} u \in \mathcal{P}_{2 p}(\widehat{T})$.

(vii) Let $V \neq(1,0)$ be a vertex of $\widehat{T}$. Then

$$
\left\|d_{V}^{-1 / 2} \mathcal{A}_{\widehat{S}} u\right\|_{L^{2}(\widehat{T})} \lesssim\|u\|_{L^{2}(\widehat{S})} \quad \text { and } \quad\left\|d_{V}^{-1 / 2} \nabla \mathcal{A}_{\widehat{S}} u\right\|_{L^{2}(\widehat{T})} \lesssim\|u\|_{H^{1}(\widehat{S})}
$$

Proof. We define the operator $\widetilde{\mathcal{A}}_{\widehat{S}}$ as

$$
\widetilde{\mathcal{A}}_{\widehat{S}}:=\mathcal{A}_{0}^{(1,0)} \circ \mathcal{R}_{\widehat{T}}
$$

where $\mathcal{R}_{\widehat{T}}$ denotes the restriction operator to the triangle $\widehat{T}$ and $\mathcal{A}_{0}^{(1,0)}$ is the operator from Lemma 5.4 with $\widehat{V}=(1,0)$. As $\mathcal{R}_{\widehat{T}} \mathcal{Q}_{p}(\widehat{S}) \subset \mathcal{P}_{2 p}(\widehat{T})$, we obtain (vi). The properties (ii) and (iii) are direct consequence of Lemma 5.4 and the properties of the restriction operator. For (iii), we note that on $\widehat{T}$ the restriction operator is the identity. Lemma 5.4 then yields

$$
\left\|d_{(1,0)}^{-\theta}\left(u-\widetilde{\mathcal{A}}_{\widehat{S}} u\right)\right\|_{L^{2}(\widehat{T})} \lesssim\left\|\mathcal{R}_{\widehat{T}} u\right\|_{H^{\theta}(\widehat{T})} \lesssim\|u\|_{H^{\theta}(\widehat{S})} .
$$

Lemma 5.4 also yields (iv). What is left to do, is to ensure (v). To that end, we introduce the notation $v^{\text {flip }}(x, y)=v(1-y, 1-x)$ and set

$$
\mathcal{A}_{\widehat{S}} u:=\frac{\widetilde{\mathcal{A}}_{\widehat{S}} u+\left(\widetilde{\mathcal{A}}_{\widehat{S}} u\right)^{\text {flip }}}{2} .
$$

The operator $\mathcal{A}_{\widehat{S}}$ clearly fulfills (ii), (iii), and (iv). To show (iii), note first that

$$
\begin{aligned}
\left\|d_{(1,0)}^{-\theta}\left(u-\mathcal{A}_{\widehat{S}} u\right)\right\|_{L^{2}(\widehat{T})} & \lesssim\left\|d_{(1,0)}^{-\theta}\left(u-\widetilde{\mathcal{A}}_{\widehat{S}} u\right)\right\|_{L^{2}(\widehat{T})}+\left\|d_{(1,0)}^{-\theta}\left(u-\left(\widetilde{\mathcal{A}}_{\widehat{S}} u\right)^{\mathrm{flip}}\right)\right\|_{L^{2}(\widehat{T})} \\
& =\left\|d_{(1,0)}^{-\theta}\left(u-\widetilde{\mathcal{A}}_{\widehat{S}} u\right)\right\|_{L^{2}(\widehat{T})}+\left\|d_{(1,0)}^{-\theta}\left(u^{\mathrm{flip}}-\widetilde{\mathcal{A}}_{\widehat{S}} u\right)\right\|_{L^{2}(\widehat{T})} \\
& \lesssim\left\|d_{(1,0)}^{-\theta}\left(u-\widetilde{\mathcal{A}}_{\widehat{S}} u\right)\right\|_{L^{2}(\widehat{T})}+\left\|d_{(1,0)}^{-\theta}\left(u-u^{\mathrm{flip}}\right)\right\|_{L^{2}(\widehat{T})} .
\end{aligned}
$$

The first term on the right-hand side is bounded by (5.19), and it suffices to bound the second term. As

$$
|(x, y)-(1-y, 1-x)| \leq 2 d_{(1,0)}(x, y),
$$


a simple argument based on the fundamental theorem of calculus and Hardy's inequality shows that

$$
\left\|d_{(1,0)}^{-\theta}\left(u-u^{\text {flip }}\right)\right\|_{L^{2}(\widehat{T})} \leq\|u\|_{H^{\theta}(\widehat{T})}
$$

for $\theta=1$. The same estimate for $\theta=0$ is trivial. An interpolation argument finishes the proof. The property (vii) also follows from Lemma 5.4 .

In order to preserve the polynomial degree also for quadrilaterals, one can modify the construction of $\mathcal{A}_{\widehat{S}}$. The only concession one needs to make is the restriction of the domain of definition.

Corollary 5.8. If $p \geq 2$, one can define $\mathcal{A}_{\widehat{S}}^{p}: \mathcal{Q}_{p}(\widehat{S}) \rightarrow \mathcal{P}_{p}(\widehat{T})$, preserving the properties from Lemma 5.7.

Proof. We can mimic the proof of Lemma 5.7, defining

$$
\widetilde{\mathcal{A}}_{\widehat{S}}^{p}:=\mathcal{A}_{0}^{(1,0)} \circ \mathcal{R}_{\widehat{T}} \circ i_{\lfloor p / 2\rfloor} .
$$

As $\mathcal{Q}_{\lfloor p / 2\rfloor}(\widehat{T}) \subseteq \mathcal{P}_{p}(\widehat{T})$, we see that $\mathcal{A}_{\widehat{S}}^{p}$ maps $\mathcal{Q}_{p}(\widehat{S})$ to $\mathcal{P}_{p}(\widehat{T})$. To show boundedness of $\mathcal{A}_{\widehat{S}}$, we additionally use the boundedness of the Gauß-Lobatto interpolation operator (see Lemma 5.6). Note also that $(1,0)$ is a Gauß-Lobatto node so that $i_{\lfloor p / 2\rfloor}$ reproduces point values. Estimate (5.19) then becomes

$$
\begin{aligned}
& \left\|d_{(1,0)}^{-\theta}\left(u-\widetilde{\mathcal{A}}_{\widehat{S}}^{p} u\right)\right\|_{L^{2}(\widehat{T})}=\left\|d_{(1,0)}^{-\theta}\left(u-\mathcal{A}_{0}^{(1,0)} \circ \mathcal{R}_{\widehat{T}} \circ i_{\lfloor p / 2\rfloor} u\right)\right\|_{L^{2}(\widehat{T})} \\
& \leq\left\|d_{(1,0)}^{-\theta}\left(u-\mathcal{A}_{0}^{(1,0)} u\right)\right\|_{L^{2}(\widehat{T})}+\left\|d_{(1,0)}^{-\theta} \mathcal{A}_{0}^{(1,0)}\left(u-i_{\lfloor p / 2\rfloor} u\right)\right\|_{L^{2}(\widehat{T})}
\end{aligned}
$$

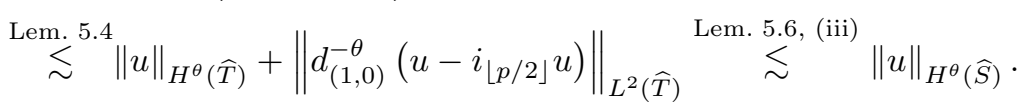

\subsection{Averaging operators for the edge parts}

In this section, we develop averaging operators on the reference triangle that reproduce values on one of the edges and produces functions which vanish on the others. They will be crucial for defining the edge contributions of the decomposition. We will need an averaging operator for edges.

Lemma 5.9. Let $\widehat{e}$ be an edge of $\widehat{T}$. Then there exists a linear operator $\mathcal{A}^{\widehat{e}}: L_{\text {loc }}^{1}(\widehat{T}) \rightarrow C^{\infty}(\widehat{T})$ with the following properties:

(i) If $u$ is a polynomial of degree $p \geq 0$, then $\mathcal{A}^{\widehat{e}} u$ is a polynomial of degree $p$.

(ii) If $u$ is continuous at a point $z \in \widehat{e}$, then $\left(\mathcal{A}^{\widehat{e}} u\right)(z)=u(z)$.

(iii) $\mathcal{A}^{\widehat{e}}: H^{\theta}(\widehat{T}) \rightarrow H^{\theta}(\widehat{T})$ is bounded for all $\theta \in[0,1]$.

(iv) For every $T^{\prime} \subset \widehat{T}$ with $\operatorname{dist}\left(T^{\prime}, \overline{\widehat{e}}\right)>0$ there exists $C_{T^{\prime}}$ such that $\left\|\mathcal{A}^{\widehat{e}} u\right\|_{L^{\infty}\left(T^{\prime}\right)} \leq C_{T^{\prime}}\|u\|_{L^{2}(\widehat{T})}$.

(v) Let $e_{1}, e_{2}$ be the two other edges of $\widehat{T}$. Introduce for the two endpoints $V_{1}, V_{2}$ of $\widehat{e}$ the distance functions $d_{i}:=d_{V_{i}}$. Then for $i=1,2$ :

$$
\begin{aligned}
\left\|d_{i}^{1 / 2} \mathcal{A}^{\widehat{e}} u\right\|_{L^{2}\left(e_{i}\right)} & \lesssim\|u\|_{L^{2}(\widehat{T})} \\
\left\|d_{i}^{-1 / 2} \mathcal{A}^{\widehat{e}} u\right\|_{L^{2}\left(e_{i}\right)} & \lesssim\left\|d_{i}^{-1} u\right\|_{L^{2}(\widehat{T})} \\
\left\|d_{i}^{1 / 2} \nabla \mathcal{A}^{\widehat{e}} u\right\|_{L^{2}\left(e_{i}\right)} & \lesssim\|\nabla u\|_{L^{2}(\widehat{T})}
\end{aligned}
$$

(vi) There holds

$$
\left\|d_{\widehat{e}}^{-1}\left(u-\mathcal{A}^{\widehat{e}} u\right)\right\|_{L^{2}(\widehat{T})} \lesssim\|\nabla u\|_{L^{2}(\widehat{T})} .
$$

Proof. Structurally, the procedure is similar to that in the proof of Lemma 5.3 . To keep the notation simple, we use the reference triangle $\widetilde{T}:=\{(\xi, \eta):-1<\xi<1, \quad 0<\eta<1-|\xi|\}$ and suppose $\widehat{e}:=(-1,1) \times\{0\}$. Let $M=\mathbb{R} \times\{0\} \supset \widehat{e}$. Select $\rho$ with $\operatorname{supp} \rho \subset \alpha(\widetilde{T}-(0,1))$ for some $\alpha \in(0,1 / \sqrt{2})$. Note that this implies supp $\rho \subset B_{1}(0)$ and $\operatorname{supp} \rho_{d_{M}(x)}(x-\cdot) \subset \widetilde{T}$ for any $x \in \widetilde{T}$. Again, with the aid of Stein's extension operator [Ste70, Sec. VI] we may assume that $u$ is defined on $\mathbb{R}^{d}$. Items (ii)-(iv) and (vil) follow from 
Lemma 5.2. In order to derive Item ( $\mathbb{\nabla})$, localized versions of the trace inequalities and covering arguments show for sufficiently smooth functions $v$

$$
\begin{aligned}
\left\|d_{i}^{1 / 2} v\right\|_{L^{2}\left(\widehat{e}_{i}\right)} & \lesssim\|v\|_{L^{2}(\widetilde{T})}+\left\|d_{\widehat{e}} \nabla v\right\|_{L^{2}(\widetilde{T})}, \\
\left\|d_{i}^{-1 / 2} v\right\|_{L^{2}\left(\widehat{e}_{i}\right)} & \lesssim\left\|d_{\widehat{e}}^{-1} v\right\|_{L^{2}(\widetilde{T})}+\|\nabla v\|_{L^{2}(\widetilde{T})}, \\
\left\|d_{i}^{1 / 2} \nabla v\right\|_{L^{2}\left(\widehat{e}_{i}\right)} & \lesssim\|\nabla v\|_{L^{2}(\widetilde{T})}+\left\|d_{\widehat{e}} \nabla^{2} v\right\|_{L^{2}(\widetilde{T})} .
\end{aligned}
$$

Applying the corresponding estimates from Lemma 5.2 finishes the proof.

The operator $\mathcal{A}^{\widehat{e}}$ can be modified so as to enforce homogeneous boundary conditions on $\partial \widehat{T} \backslash \widehat{e}$ :

Lemma 5.10. Let $d_{1}$ and $d_{2}$ be the distances from the two vertices of $\widehat{e}$. There exists a linear operator $\mathcal{A}_{0}^{\widehat{e}}: L_{\text {loc }}^{1}(\widehat{T}) \rightarrow C^{\infty}(\widehat{T})$ with the following properties:

(i) $\mathcal{A}_{0}^{\widehat{e}}$ is bounded in $L^{2}(\widehat{T})$.

(ii) For each $\theta \in[0,1]$ there is $C>0$ such that, provided the right-hand side is finite,

$$
\left\|\mathcal{A}_{0}^{\widehat{e}} u\right\|_{H^{\theta}(\widehat{T})} \leq C\left[\|u\|_{H^{\theta}(\widehat{T})}+\left\|d_{1}^{-\theta} u\right\|_{L^{2}(\widehat{T})}+\left\|d_{2}^{-\theta} u\right\|_{L^{2}(\widehat{T})}\right] .
$$

(iii) $\left.\left(\mathcal{A}_{0}^{\widehat{e}} u\right)\right|_{\partial \widehat{T} \backslash \widehat{e}}=0$.

(iv) If $u$ is continuous at $z \in \widehat{e}$, then $\left(\mathcal{A}_{0}^{\widehat{e}} u\right)(z)=u(z)$.

(v) If $u$ is a polynomial degree $p$ and $u$ vanishes in the endpoints of $\widehat{e}$, then $\mathcal{A}_{0}^{\widehat{e}} u$ is a polynomial of degree $p$.

(vi) It holds

$$
\left\|d_{\widehat{e}}^{-\theta}\left(u-\mathcal{A}_{0}^{\widehat{e}} u\right)\right\|_{L^{2}(\widehat{T})} \leq C\|u\|_{H^{\theta}(\widehat{T})}+\left\|d_{1}^{-\theta} u\right\|_{L^{2}(\widehat{T})}+\left\|d_{2}^{-\theta} u\right\|_{L^{2}(\widehat{T})} .
$$

(vii) Let $\widehat{V}$ be the vertex opposite of $\widehat{e}$. Then

$$
\begin{aligned}
\left\|d_{\widehat{V}}^{-1 / 2} \mathcal{A}_{0}^{\widehat{e}} u\right\|_{L^{2}(\widehat{T})} & \lesssim\|u\|_{L^{2}(\widehat{T})} \quad \text { and } \\
\left\|d_{\widehat{V}}^{-1 / 2} \nabla \mathcal{A}_{0}^{\widehat{e}} u\right\|_{L^{2}(\widehat{T})} & \lesssim\|u\|_{H^{1}(\widehat{T})}+\left\|d_{1}^{-1} u\right\|_{L^{2}(\widehat{T})}+\left\|d_{2}^{-1} u\right\|_{L^{2}(\widehat{T})} .
\end{aligned}
$$

Proof. We assume $\widehat{V}=(1,1)$ and $\widehat{e}=\{(x, 0) \mid 0<x<1\}$. We construct $\mathcal{A}_{0}^{\widehat{e}}$ explicitly with the aid of $\mathcal{A}^{\widehat{e}}$. For simplicity of notation, we write $u_{1}:=\mathcal{A}^{\widehat{e}} u$. As a first step, we set $u_{2}:=u_{1}-y u_{1}(1,1)$. Then $u_{2}$ vanishes in $(1,1)$ and $u_{2}$ has the desired stability properties in $L^{2}$ and $H^{1}$ by those of $\mathcal{A}^{\widehat{e}} u$ given in Lemma [5.9. Next, we correct the behavior of $u_{2}$ on the edge $\widehat{e}_{1}=\{(x, x) \mid x \in(0,1)\}$. We set

$$
u_{3}(\xi, \eta):=u_{2}(\xi, \eta)-\frac{\eta}{\xi} u_{2}(\xi, \xi)
$$

Then $u_{3}$ coincides with $u_{2}$ on $\widehat{e}$, it coincides with $u_{2}$ on $\widehat{e}_{2}=\{(1, \eta) \mid 0<\eta<1\}$ in view of $u_{2}(1,1)=0$, and it vanishes on $\widehat{e}_{1}$. When trying to control the $L^{2}$-norm of $u_{3}$ the correction part of $u_{3}$ leads to having to control $\left\|d_{1}^{1 / 2} u_{2}\right\|_{L^{2}\left(\widehat{e}_{1}\right)}$, where $d_{1}$ denotes the distance from $V_{1}=(0,0)$ This is estimated by $\|u\|_{L^{2}(\widehat{T})}$ in view of (5.20) in Lemma 5.9, For the $H^{1}$-norm, the correction part of $u_{3}$ leads to several contributions which can all be controlled by Lemma [5.9, (v). In particular, it is responsible for a term $\left\|d_{1}^{-1 / 2} u_{2}\right\|_{L^{2}\left(\widehat{e}_{1}\right)}$, which can be estimated by $\left\|d_{1}^{-1} u\right\|_{L^{2}(\widehat{T})}$ by (5.21), and a term $\left\|d_{1}^{1 / 2} \nabla u_{2}\right\|_{L^{2}\left(\widehat{e}_{1}\right)}$, which is controlled by (5.22). The intermediate cases $\theta \in(0,1)$ follow by interpolation using Lemma 4.7. We still need to show the weighted $L^{2}$ estimate (vil). We again restrict ourselves to the case $\theta=1$, the general result follows via interpolation using Lemma 4.7. Since $u_{2}$ satisfies this estimate, we only need to estimate the correction term. A simple calculation yields a term of the form $\left\|d_{1}^{-1 / 2} u_{2}\right\|_{L^{2}\left(\widehat{e}_{1}\right)}$, which can again be controlled by $\left\|d_{1}^{-1} u\right\|_{L^{2}(\widehat{T})}$ via (5.22). Next, a correction for the edge $e_{2}$ is performed that is completely analogous to that for the edge $\widehat{e}_{1}$. This produces the final function $u_{4}=: \mathcal{A}_{0}^{\widehat{e}} u$. It remains to see that $u_{4}$ is a polynomial if $u$ is a polynomial that vanishes in the two endpoints of $\widehat{e}$. This follows by inspection and the fact that $\mathcal{A}^{\widehat{e}} u$ is a polynomial if $u$ is a polynomial and that $\left.\left(\mathcal{A}^{\widehat{e}} u\right)\right|_{\widehat{e}}=\left.u\right|_{\widehat{e}}$. The property (viil) is shown analogously as before. 


\section{Decomposition of FEM spaces}

In this section, we use the averaging operators defined on the reference elements to construct the vertex-, edge- and element contributions on the respective patches. The basic structure is to single out from the patch one element on which to perform the averaging. In the discrete setting, this should be the element with lowest polynomial degree. The averaged function on that element is then extended to the whole patch by "rotation".

\subsection{The vertex parts}

Lemma 6.1. Fix $V \in \mathcal{V}^{\text {int }}$ and let $\omega_{V}$ be its vertex patch as defined in Definition 2.2. Then there exists an operator $\mathcal{A}_{\omega_{V}}: L^{2}(\Gamma) \rightarrow L^{2}\left(\omega_{V}\right)$ with the following properties and implied constants depending only on $\Gamma$ and the shape-regularity of $\mathcal{T}$ :

(i) For all $\theta \in[0,1]$, there holds $\left\|\mathcal{A}_{\omega_{V}} u\right\|_{\widetilde{H}_{h}^{\theta}\left(\omega_{V}\right)} \lesssim\|u\|_{H_{h}^{\theta}\left(\omega_{V}\right)}$.

(ii) The following weighted norm estimates hold:

$$
\begin{aligned}
\left\|d_{V}^{-\theta}\left(u-\mathcal{A}_{\omega_{V}} u\right)\right\|_{L^{2}\left(\omega_{V}\right)} & \lesssim\|u\|_{H_{h}^{\theta}\left(\omega_{V}\right)}, \\
\left\|d_{V^{\prime}}^{-\theta} \mathcal{A}_{\omega_{V}} u\right\|_{L^{2}\left(\omega_{V}\right)} & \lesssim\|u\|_{H_{h}^{\theta}\left(\omega_{V}\right)} \quad \text { for vertices } V^{\prime} \neq V .
\end{aligned}
$$

(iii) If $\left.\mathcal{T}\right|_{\omega_{V}}$ consists of triangles only, then $\mathcal{A}_{\omega_{V}}: \widetilde{\mathcal{S}}^{\mathbf{p}, 1}(\mathcal{T}) \rightarrow \widetilde{\mathcal{S}}^{\mathbf{p}, 1}\left(\left.\mathcal{T}\right|_{\omega_{V}}\right)$. If $\left.\mathcal{T}\right|_{\omega_{V}}$ consists of triangles and quadrilaterals, then $\mathcal{A}_{\omega_{V}}: \widetilde{\mathcal{S}}^{\mathbf{p}, 1}(\mathcal{T}) \rightarrow \widetilde{\mathcal{S}}^{2 \mathbf{p}, 1}\left(\left.\mathcal{T}\right|_{\omega_{V}}\right)$.

Proof. Select an element $K \subset \omega_{V}$ with the lowest polynomial degree. Let $\widehat{K}$ be the corresponding reference element with element map $F_{K}$, with (for notational convenience) the additional assumption that $F_{K}(1,0)=$ $V$. Depending on whether $K$ is a triangle $\widehat{K}=\widehat{T}$ or square $\widehat{K}=\widehat{S}$, we define

$$
\widetilde{u}:= \begin{cases}\mathcal{A}_{0}^{(1,0)} \widehat{u} & \text { if } \widehat{K}=\widehat{T}, \\ \mathcal{A}_{\widehat{S}} \widehat{u} & \text { if } \widehat{K}=\widehat{S},\end{cases}
$$

where $\widehat{u}:=u \circ F_{K}$ denotes the pullback of $u$ to the reference element and $\mathcal{A}_{\widehat{S}}$ is the operator from Lemma 5.7 For all elements $K^{\prime} \subset \omega_{V}$, let $F_{K^{\prime}}: \widehat{K^{\prime}} \rightarrow K^{\prime}$ denote the element map, with the additional assumption that $F_{K^{\prime}}(1,0)=V$. We define $\mathcal{A}_{\omega_{V}}$ by "rotating" $\widetilde{u}$, i.e.,

$$
\left.\left(\mathcal{A}_{\omega_{V}} u\right)\right|_{K^{\prime}}:= \begin{cases}\widetilde{u} \circ F_{K^{\prime}}^{-1} & \text { if } \widehat{K^{\prime}}=\widehat{T}, \\ (\mathcal{D} \widetilde{u}) \circ F_{K^{\prime}}^{-1} & \text { if } \widehat{K^{\prime}}=\widehat{S} .\end{cases}
$$

If $K^{\prime}$ is a triangle, scaling arguments and Lemma 5.4 (if $K$ is a triangle) or Lemma 5.7 (if $K$ is a quadrilateral) show for $\theta \in\{0,1\}$

$$
\left\|\mathcal{A}_{\omega_{V}} u\right\|_{H_{h}^{\theta}\left(K^{\prime}\right)} \lesssim\|u\|_{H_{h}^{\theta}(K)} .
$$

If $K^{\prime}$ is a quadrilateral, we additionally use boundedness of the Duffy operator $\mathcal{D}$ from Lemma [5.5, (i),

$$
\begin{aligned}
\left\|\mathcal{A}_{\omega_{V}} u\right\|_{L^{2}\left(K^{\prime}\right)} & \lesssim h_{K^{\prime}}\|\mathcal{D} \widetilde{u}\|_{L^{2}(\widehat{S})} \lesssim h_{K^{\prime}}\left\|d_{(1,1)}^{-1 / 2} \widetilde{u}\right\|_{L^{2}(\widehat{T})} \lesssim\|u\|_{L^{2}(K)}, \\
\left\|\mathcal{A}_{\omega_{V}} u\right\|_{H_{h}^{1}\left(K^{\prime}\right)} & \lesssim\|\mathcal{D} \widetilde{u}\|_{H^{1}(\widehat{S})} \lesssim\left\|d_{(1,1)}^{-1 / 2} \widetilde{u}\right\|_{L^{2}(\widehat{T})}+\left\|d_{(1,1)}^{-1 / 2} \nabla \widetilde{u}\right\|_{L^{2}(\widehat{T})} \lesssim\|u\|_{H_{h}^{1}(K)} .
\end{aligned}
$$

The properties of Lemmas 5.7, ( $\mathrm{V}$ ) and 5.4 (ix) ensure global continuity of $\mathcal{A}_{\omega_{V}} u$. The Duffy transform maps the edge $e_{1}:=\{0\} \times(0,1)$ to the edge $e:=\{(x, x), 0<x<1\}$, and the edge $e_{2}:=(0,1) \times\{1\}$ to the vertex $(1,1)$. As $\widetilde{u}$ vanishes on $e$ and at $(1,1)$, we get that $\mathcal{A}_{\omega_{V}} u$ vanishes on all of $\partial \omega_{V}$. Summing the estimates (6.3) and (6.4) over all elements $K^{\prime}$ of the patch $\omega_{V}$, we get that the operator $\mathcal{A}_{\omega_{V}}$ is bounded as

$$
L^{2}(K) \rightarrow L^{2}\left(\omega_{V}\right) \quad \text { and } \quad H_{h}^{1}(K) \rightarrow \widetilde{H}_{h}^{1}\left(\omega_{V}\right) .
$$


An interpolation argument yields (ii). It remains to show (iii). Using Lemma 4.9 and the statement (ii) of the current lemma, we obtain

$$
\left\|d_{V}^{-\theta}\left(u-\mathcal{A}_{\omega_{V}} u\right)\right\|_{L^{2}\left(\omega_{V}\right)} \lesssim\left\|d_{V}^{-\theta}\left(u-\mathcal{A}_{\omega_{V}} u\right)\right\|_{L^{2}(K)}+\|u\|_{H_{h}^{\theta}\left(\omega_{V}\right)}
$$

It remains to bound the first term on the right-hand side. For notational simplicity assume $\widehat{K}=\widehat{S}$, the case of $\widehat{K}=\widehat{T}$ follows along the same lines but is even simpler, as the Duffy transform can be omitted. A scaling argument and the triangle inequality yield

$$
\left\|d_{V}^{-\theta}\left(u-\mathcal{A}_{\omega_{V}} u\right)\right\|_{L^{2}(K)} \lesssim h_{K}^{1-\theta}\left(\left\|d_{(1,0)}^{-\theta}(\widehat{u}-\mathcal{D} \widetilde{u})\right\|_{L^{2}(\widehat{T})}+\left\|d_{(1,0)}^{-\theta}(\widehat{u}-\mathcal{D} \widetilde{u})\right\|_{L^{2}(\widehat{S} \backslash \widehat{T})}\right) .
$$

Since $d_{(1,0)}$ is bounded uniformly from below on $\widehat{S} \backslash \widehat{T}$, the same arguments as in (6.4) show

$$
\left\|d_{(1,0)}^{-\theta}(\widehat{u}-\mathcal{D} \widetilde{u})\right\|_{L^{2}(\widehat{S} \backslash \widehat{T})} \lesssim\|\widehat{u}\|_{L^{2}(\widehat{S} \backslash \widehat{T})}+\|\mathcal{D} \widetilde{u}\|_{L^{2}(\widehat{S} \backslash \widehat{T})} \lesssim\|\widehat{u}\|_{L^{2}(\widehat{S})} \lesssim h_{K}^{-1}\|u\|_{L^{2}(K)},
$$

where we have used the boundedness of $\mathcal{D}$ and $\mathcal{A}_{\widehat{S}}$. Using the same arguments, we see

$$
\begin{aligned}
\left\|d_{(1,0)}^{-\theta}(\widehat{u}-\mathcal{D} \widetilde{u})\right\|_{L^{2}(\widehat{T})} & \leq\left\|d_{(1,0)}^{-\theta}(\widehat{u}-\widetilde{u})\right\|_{L^{2}(\widehat{T})}+\left\|d_{(1,0)}^{-\theta}(\widetilde{u}-\mathcal{D} \widetilde{u})\right\|_{L^{2}(\widehat{T})} \\
& \stackrel{5.15}{\lesssim}\left\|d_{(1,0)}^{-\theta}(\widehat{u}-\widetilde{u})\right\|_{L^{2}(\widehat{T})}+\|\widetilde{u}\|_{H^{\theta}(\widehat{T})}+\left\|d_{(1,1)}^{-1 / 2} \widetilde{u}\right\|_{L^{2}(\widehat{T})} \\
& \lesssim\|\widehat{u}\|_{H^{\theta}(\widehat{T})} .
\end{aligned}
$$

Combining these estimates and using Lemma 4.1 gives $\left\|d_{V}^{-\theta}\left(u-\mathcal{A}_{\omega_{V}} u\right)\right\|_{L^{2}(K)} \lesssim\|u\|_{H_{h}^{\theta}(K)}$.

To show (6.2), we again only consider $\theta=1$. On the elements $K^{\prime}$ with $\operatorname{dist}\left(V^{\prime}, K\right)>0$ the estimate is just $L^{2}$ stability, therefore we may only consider elements $K^{\prime}$ with $V^{\prime} \in \overline{K^{\prime}}$. We note that $V^{\prime} \in \overline{\omega_{V}}$ but $V \neq V^{\prime}$ implies that there exists an edge $e \subset \partial \omega_{V} \cap \overline{K^{\prime}}$ with $V^{\prime} \in \bar{e}$. By construction, $\mathcal{A}_{\omega_{V}} u$ vanishes on $e$. Transforming to the reference element and applying Lemma 4.4 gives the stated estimate.

Property (iii) follows immediately from Lemmas 5.4 and 5.7 and the fact that the Duffy transform maps $\mathcal{P}_{p}(\widehat{T}) \rightarrow \mathcal{Q}_{p}(\widehat{S})$.

If $\mathcal{T}$ consists of triangles and quadrilaterals, an operator analogous to $\mathcal{A}_{\omega_{V}}$ from Lemma 6.1 can be defined which preserves the polynomial degree.

Corollary 6.2. There exists an operator $\mathcal{A}_{\omega_{V}}^{p}: \widetilde{\mathcal{S}}^{\mathbf{p}, 1}(\mathcal{T}) \rightarrow \widetilde{\mathcal{S}}^{\mathbf{p}}, 1\left(\left.\mathcal{T}\right|_{\omega_{V}}\right)$, retaining the stability properties of Lemma 6.1

Proof. We can mimic the proof of Lemma 6.1. For the definition of $\widetilde{u}$, we use the operator $\mathcal{A}_{\widehat{S}}^{p}$ from Corollary 5.8. Then, if $K$ happens to be a square, instead of (6.5) we get that $\mathcal{A}_{\omega_{V}}^{p}$ is bounded as

$$
\left(\mathcal{Q}_{p}(K),\|\cdot\|_{L^{2}(K)}\right) \rightarrow L^{2}\left(\omega_{V}\right) \quad \text { and } \quad\left(\mathcal{Q}_{p}(K),\|\cdot\|_{H_{h}^{1}(K)}\right) \rightarrow \widetilde{H}_{h}^{1}\left(\omega_{V}\right)
$$

An interpolation argument and Lemma 5.6 (ii) show the result.

\subsection{The edge parts}

Lemma 6.3. Let $\omega_{e}$ denote an edge patch. Then there exists an operator $\mathcal{A}_{\omega_{e}}: L^{2}(\Gamma) \rightarrow L^{2}\left(\omega_{e}\right)$ with the following properties:

(i) For all $\theta \in[0,1]$ and $d_{1}$ and $d_{2}$ denoting the distances to the endpoints of e, there holds (provided the right-hand side is finite)

$$
\left\|\mathcal{A}_{\omega_{e}} u\right\|_{\widetilde{H}_{h}^{\theta}\left(\omega_{e}\right)} \lesssim\|u\|_{H_{h}^{\theta}\left(\omega_{e}\right)}+\left\|d_{1}^{-\theta} u\right\|_{L^{2}\left(\omega_{e}\right)}+\left\|d_{2}^{-\theta} u\right\|_{L^{2}\left(\omega_{e}\right)}
$$


(ii) For all $\theta \in[0,1]$ and all other edges $e^{\prime} \subset \overline{\omega_{e}}$ there holds (provided the right-hand sides are finite)

$$
\begin{aligned}
\left\|d_{e}^{-\theta}\left(u-\mathcal{A}_{\omega_{e}} u\right)\right\|_{L^{2}\left(\omega_{e}\right)} & \lesssim\|u\|_{H_{h}^{\theta}\left(\omega_{e}\right)}+\left\|d_{1}^{-\theta} u\right\|_{L^{2}\left(\omega_{e}\right)}+\left\|d_{2}^{-\theta} u\right\|_{L^{2}\left(\omega_{e}\right)}, \\
\left\|d_{e^{\prime}}^{-\theta} \mathcal{A}_{\omega_{e}} u\right\|_{L^{2}\left(\omega_{e}\right)} & \lesssim\|u\|_{H_{h}^{\theta}\left(\omega_{e}\right)}+\left\|d_{1}^{-\theta} u\right\|_{L^{2}\left(\omega_{e}\right)}+\left\|d_{2}^{-\theta} u\right\|_{L^{2}\left(\omega_{e}\right)} .
\end{aligned}
$$

(iii) Let $u \in \widetilde{\mathcal{S}}^{\mathbf{p}, 1}(\mathcal{T})$ vanish in all vertices of $\omega_{e}$. If $\left.\mathcal{T}\right|_{\omega_{e}}$ consists of triangles only then $\mathcal{A}_{\omega_{e}} u \in \widetilde{\mathcal{S}} \mathbf{p}, 1\left(\left.\mathcal{T}\right|_{\omega_{e}}\right)$. If $\left.\mathcal{T}\right|_{\omega_{e}}$ consists of triangles and quadrilaterals, then $\mathcal{A}_{\omega_{e}} u \in \widetilde{\mathcal{S}}^{2 \mathbf{p}, 1}\left(\left.\mathcal{T}\right|_{\omega_{e}}\right)$.

Proof. Let $K$ be the element of $\omega_{e}$ with the lowest polynomial degree, and $K^{\prime}$ the other element of $\omega_{e}$. Suppose that the corresponding element maps fulfill $F_{K}(\widehat{e})=F_{K^{\prime}}(\widehat{e})=e$, where $\widehat{e}:=(0,1) \times\{0\}$, but $F_{K}$ and $F_{K^{\prime}}$ having different orientation on $e$. We distinguish four cases:

(1) $K$ is a triangle, i.e., $\widehat{K}=\widehat{T}$ : Define $\widetilde{u}:=\mathcal{A}_{0}^{\widehat{e}}\left(u \circ F_{K}\right),\left.\left(\mathcal{A}_{\omega_{e}} u\right)\right|_{K}:=\widetilde{u} \circ F_{K}^{-1}$, and

$$
\left.\left(\mathcal{A}_{\omega_{e}} u\right)\right|_{K^{\prime}}:= \begin{cases}\widetilde{u} \circ F_{K^{\prime}}^{-1} & \text { if } \widehat{K^{\prime}}=\widehat{T} \\ (\mathcal{D} \widetilde{u}) \circ F_{K^{\prime}}^{-1} & \text { if } \widehat{K^{\prime}}=\widehat{S} .\end{cases}
$$

(2) $K$ is a square, $K^{\prime}$ is a triangle, and $p_{K} \leq p_{K^{\prime}} \leq 2 p_{K}$ : Define $\widetilde{u}:=\mathcal{A}_{0}^{\widehat{e}}\left(u \circ F_{K^{\prime}}\right)$ and $\left.\left(\mathcal{A}_{\omega_{e}} u\right)\right|_{K^{\prime}}:=$ $\widetilde{u} \circ F_{K^{\prime}}^{-1}$. On $K$, define $\left.\left(\mathcal{A}_{\omega_{e}} u\right)\right|_{K}:=(\mathcal{D} \widetilde{u}) \circ F_{K}^{-1}$.

(3) $K$ is a square, $K^{\prime}$ is a triangle, and $p_{K^{\prime}}>2 p_{K}$ : Define $\widetilde{u}:=\mathcal{A}_{0}^{\widehat{e}}\left(u \circ F_{K}\right),\left.\left(\mathcal{A}_{\omega_{e}} u\right)\right|_{K^{\prime}}:=\widetilde{u} \circ F_{K^{\prime}}^{-1}$, and $\left.\left(\mathcal{A}_{\omega_{e}} u\right)\right|_{K}:=(\mathcal{D} \widetilde{u}) \circ F_{K}^{-1}$

(4) $K$ and $K^{\prime}$ are squares: Define $\widetilde{u}:=\mathcal{A}_{0}^{\widehat{e}}\left(u \circ F_{K}\right),\left.\left(\mathcal{A}_{\omega_{e}} u\right)\right|_{K^{\prime}}:=(\mathcal{D} \widetilde{u}) \circ F_{K^{\prime}}^{-1}$, and $\left.\left(\mathcal{A}_{\omega_{e}} u\right)\right|_{K}:=$ $(\mathcal{D} \widetilde{u}) \circ F_{K}^{-1}$.

Let us show the boundedness in case (1), the other cases follow by the same argument. First, writing $\widehat{u}:=u \circ F_{K}$, scaling arguments, and Lemma 5.10, (iii), show for $\theta \in\{0,1\}$

$$
\begin{aligned}
\left\|\mathcal{A}_{\omega_{e}} u\right\|_{H_{h}^{\theta}(K)} & \lesssim h_{K}^{1-\theta}\left(\| \mathcal{A}_{0}^{\widehat{e} \widehat{u} \|_{L^{2}(\widehat{T})}}+\left|\mathcal{A}_{0}^{\widehat{e} \widehat{u}}\right|_{H^{\theta}(\widehat{T})}\right) \lesssim h_{K}^{1-\theta}\left(\|\widehat{u}\|_{H^{\theta}(\widehat{K})}+\left\|d_{\widehat{1}}^{-\theta} \widehat{u}\right\|_{L^{2}(\widehat{K})}+\left\|d_{\widehat{2}}^{-\theta} \widehat{u}\right\|_{L^{2}(\widehat{K})}\right) \\
& \lesssim\|u\|_{H_{h}^{\theta}(K)}+\left\|d_{1}^{-\theta} u\right\|_{L^{2}(K)}+\left\|d_{2}^{-\theta} u\right\|_{L^{2}(K)} .
\end{aligned}
$$

If $K^{\prime}$ is a triangle, the same estimate holds with $K^{\prime}$ instead of $K$ on the left-hand side. If $K^{\prime}$ is a square, we additionally invoke Lemma 5.5] (ii), and Lemma 5.10, (viii), to show for $\theta=1$

$$
\begin{aligned}
\left\|\mathcal{A}_{\omega_{e}} u\right\|_{H_{h}^{\theta}\left(K^{\prime}\right)} & \lesssim h_{K}^{1-\theta}\left(\left\|\mathcal{D} \mathcal{A}_{0}^{\widehat{e} \widehat{u}}\right\|_{L^{2}(\widehat{S})}+\left|\mathcal{D} \mathcal{A}_{0}^{\widehat{e} \widehat{u}}\right|_{H^{\theta}(\widehat{S})}\right) \\
& \lesssim h_{K}^{1-\theta}\left(\left\|d_{(1,1)}^{-1 / 2} \mathcal{A}_{0}^{\widehat{e} \widehat{u}}\right\|_{L^{2}(\widehat{T})}+\left\|d_{(1,1)}^{-1 / 2} \nabla \mathcal{A}_{0}^{\widehat{e} \widehat{u}}\right\|_{L^{2}(\widehat{T})}\right) \\
& \lesssim h_{K}^{1-\theta}\left(\|\widehat{u}\|_{H^{\theta}(\widehat{K})}+\left\|d_{\widehat{1}}^{-\theta} \widehat{u}\right\|_{L^{2}(\widehat{K})}+\left\|d_{\widehat{2}}^{-\theta} \widehat{u}\right\|_{L^{2}(\widehat{K})}\right) \\
& \lesssim\|u\|_{H_{h}^{\theta}(K)}+\left\|d_{1}^{-\theta} u\right\|_{L^{2}(K)}+\left\|d_{2}^{-\theta} u\right\|_{L^{2}(K)}
\end{aligned}
$$

The same estimate is true for $\theta=0$. These arguments can be used for the cases (2), (3), and (4). Furthermore, as $\widetilde{u}$ vanishes on $\widehat{T} \backslash \widehat{e}$, we conclude that $\mathcal{D} \widetilde{u}$ and hence $\mathcal{A}_{\omega_{e}} u$ vanish on $\widehat{S} \backslash \widehat{e}$. Summing the last estimates over all elements in $\omega_{e}$, we get that the operator $\mathcal{A}_{\omega_{e}}$ is bounded uniformly as

$$
\begin{aligned}
L^{2}(K) & \rightarrow L^{2}\left(\omega_{e}\right) \\
H_{h}^{1}\left(K, d_{1}^{-1}+d_{2}^{-1}\right) & \rightarrow \widetilde{H}_{h}^{1}\left(\omega_{e}\right) .
\end{aligned}
$$

The statement (ii) now follows by an interpolation argument and the following considerations: By Corollary 4.3 and Lemma 4.7 we have for $\theta \in[0,1]$

$$
h_{K}^{\theta-1}\|u\|_{\left(L_{2}(K), H_{h}^{1}\left(K, d_{1}^{-1}+d_{2}^{-1}\right)\right)_{\theta, 2}} \sim\|\widehat{u}\|_{\left(L_{2}(\widehat{K}), H^{1}\left(\widehat{K}, d_{\widehat{1}}^{-1}+d_{\widehat{2}}^{-1}\right)\right)_{\theta, 2}} \sim\|\widehat{u}\|_{H^{\theta}(\widehat{K})}+\left\|\left(d_{\widehat{1}}^{-\theta}+d_{\widehat{2}}^{-\theta}\right) \widehat{u}\right\|_{L^{2}(\widehat{K})},
$$


and according to Corollary 4.3 we have

$$
\|\widehat{u}\|_{H^{\theta}(\widehat{K})}+\left\|\left(d_{\widehat{1}}^{-\theta}+d_{\widehat{2}}^{-\theta}\right) \widehat{u}\right\|_{L^{2}(\widehat{K})} \sim h_{K}^{\theta-1}\left[\|u\|_{H_{h}^{\theta}(K)}+\left\|d_{1}^{-\theta} u\right\|_{L^{2}(K)}+\left\|d_{2}^{-\theta} u\right\|_{L^{2}(K)}\right] .
$$

This finishes the proof of (i) in the cases (1), (3), and (4). The same argument can be used in the case (2), only the notation $K^{\prime}$ and $K$ has to be adapted correspondingly. In all cases, $\mathcal{A}_{\omega_{e}}$ reproduces the values on $e$, i.e., $u-\mathcal{A}_{\omega_{e}} u$ vanishes on $e$, and we can use Lemma 4.4, (iii) and $1 \lesssim h_{\omega_{e}}^{-1}$ to estimate

$$
\left\|d_{e}^{-1}\left(u-\mathcal{A}_{\omega_{e}} u\right)\right\|_{L^{2}\left(\omega_{e}\right)} \lesssim\left\|u-\mathcal{A}_{\omega_{e}} u\right\|_{H_{h}^{1}\left(\omega_{e}\right)} .
$$

Therefore,

$$
\left\|d_{e}^{-\theta}\left(u-\mathcal{A}_{\omega_{e}} u\right)\right\|_{L^{2}\left(\omega_{e}\right)} \lesssim\left\|u-\mathcal{A}_{\omega_{e}} u\right\|_{H_{h}^{\theta}\left(\omega_{e}\right)},
$$

and with the triangle inequality and (ii) we conclude (iii). The proof of the estimate for $e^{\prime}$ follows along the same lines, but using that $\mathcal{A}_{\omega_{e}} u$ vanishes on $e^{\prime}$. Property (iii) follows immediately from Lemma [5.10, and the fact that the Duffy transform maps $\mathcal{P}_{p}(\widehat{T}) \rightarrow \mathcal{Q}_{p}(\widehat{S})$.

Corollary 6.4. There exists a modified operator $\mathcal{A}_{\omega_{e}}^{p}$, defined for all $u \in \widetilde{\mathcal{S}}^{\mathbf{p}, 1}(\mathcal{T})$ that vanish in all vertices of $\left.\mathcal{T}\right|_{\omega_{e}}$, such that $u$ is mapped to $\mathcal{A}_{\omega_{e}}^{p} u \in \widetilde{\mathcal{S}}^{\mathbf{p}, 1}\left(\left.\mathcal{T}\right|_{\omega_{e}}\right)$. If Assumption 2.3 holds, then the bounds from Lemma 6.3 (2i), (iii) hold. If Assumption 2.3 is violated, the bounds only hold for $\theta \in\{0,1\}$.

Proof. We mimic the proof of Lemma 6.3. In the case (1), the operators from Lemma 6.3 already have all the necessary mapping properties. In the cases (3) and (4), we define

$$
\mathcal{A}_{\omega_{e}}^{p} u:=\widetilde{\mathcal{A}}_{\omega_{e}}^{p} \circ i_{p_{K}} \circ \mathcal{D} \circ \mathcal{A}_{0}^{\widehat{e}}\left[u \circ F_{K}\right]
$$

with an auxiliary operator $\widetilde{A}_{\omega_{e}}^{p}$ on the reference square. $\widetilde{A}_{\omega_{e}}^{p} v$ is defined using $\widetilde{v}:=\mathcal{A}_{0}^{\widehat{e}} v$ and distinguishing the cases

(3) $K$ is a square, $K^{\prime}$ is a triangle, and $p_{K^{\prime}}>2 p_{K}$ : Define $\left.\left(\widetilde{A}_{\omega_{e}}^{p} v\right)\right|_{K^{\prime}}:=\widetilde{v} \circ F_{K^{\prime}}^{-1}$, and $\left.\left(\widetilde{A}_{\omega_{e}}^{p} v\right)\right|_{K}:=$ $\left(i_{p_{K}} \circ \mathcal{D} \widetilde{v}\right) \circ F_{K}^{-1}$

(4) $K$ and $K^{\prime}$ are squares: Set $\left.\left(\widetilde{A}_{\omega_{e}}^{p} v\right)\right|_{K^{\prime}}:=\left(i_{p_{K}} \circ \mathcal{D} \widetilde{v}\right) \circ F_{K^{\prime}}^{-1}$, and $\left.\left(\widetilde{A}_{\omega_{e}}^{p} v\right)\right|_{K}:=\left(i_{p_{K}} \circ \mathcal{D} \widetilde{v}\right) \circ F_{K}^{-1}$.

For functions that vanish on $\partial \widehat{S} \backslash \overline{\hat{e}}$, the operator $\widetilde{A}_{\omega_{e}}^{p}$ satisfies the following stability conditions:

$$
\left(\widetilde{\mathcal{Q}}_{p}(\widehat{S}, \partial \widehat{S} \backslash \overline{\widehat{e}}),\|\cdot\|_{L^{2}(\widehat{S})}\right) \rightarrow L^{2}\left(\omega_{e}\right) \quad \text { and } \quad\left(\widetilde{\mathcal{Q}}_{p}(\widehat{S}, \partial \widehat{S} \backslash \overline{\widehat{e}}),\|\cdot\|_{H^{1}(\widehat{S})}\right) \rightarrow \widetilde{H}_{h}^{1}\left(\omega_{e}\right)
$$

This can be seen similarly to Lemma 6.3 using the following insights:

- We additionally have to invoke the stability of the Gauß-Lobatto operator given in Lemma 5.6. (iii).

- Via Lemma 4.4 (ii), we can estimate $\left\|d_{\widehat{V}}^{-1} v\right\|_{L^{2}(\widehat{S})} \lesssim\|v\|_{H^{1}(\widehat{S})}$ for both vertices $\widehat{V}$ of $\widehat{e}$.

Interpolation of (6.7) via Lemma [5.6. (1) gives the stability $\left\|\widetilde{\mathcal{A}}_{\omega_{e}}^{p} v\right\|_{\widetilde{H}_{h}^{\theta}\left(\omega_{e}\right)} \lesssim\|v\|_{\widetilde{H}^{\theta}(\widehat{S}, \partial \widehat{S} \backslash \widehat{e})}$.

We note that the averaging $u \mapsto \mathcal{D} \circ \mathcal{A}_{0}^{\widehat{e}}\left[u \circ F_{K}\right]$ satisfies via Lemma [5.10, (iii), (vii) and Lemma 5.5,

$$
\left\|\mathcal{D} \circ \mathcal{A}_{0}^{\widehat{e}}\left[u \circ F_{K}\right]\right\|_{\widetilde{H}^{\theta}(\widehat{S}, \partial \widehat{S} \backslash \widehat{e})} \lesssim\|u\|_{H_{h}^{\theta}(K)}+\left\|d_{1}^{-\theta} u\right\|_{L^{2}(K)}+\left\|d_{2}^{-\theta} u\right\|_{L^{2}(K)}
$$

and increases the polynomial degree at most by a factor of two. This and the stability of $i_{p_{K}}$ implies that $\mathcal{A}_{\omega_{e}}$ is a stable operator satisfying the bound from Lemma 6.3. (1i).

The case (2), which is the case excluded by Assumption 2.3 needs special considerations. If $K$ is a square, $K^{\prime}$ is a triangle, and $p_{K} \leq p_{K^{\prime}} \leq 2 p_{K}$, define $\widetilde{u}:=\mathcal{A}_{0}^{e}\left(u \circ F_{K^{\prime}}\right)$ and $\left.\left(\mathcal{A}_{\omega_{e}}^{p} u\right)\right|_{K^{\prime}}:=\widetilde{u} \circ F_{K^{\prime}}^{-1}$. On $K$, define $\left.\left(\mathcal{A}_{\omega_{e}}^{p} u\right)\right|_{K}:=\left(i_{p_{K}} \circ \mathcal{D} \widetilde{u}\right) \circ F_{K}^{-1}$. This operator is then bounded in the $L^{2}$ - and $H_{h}^{1}$-norms, but the continuity of the resulting function relies on the fact that $\left.u\right|_{e} \in \mathcal{P}_{p_{K}}$, i.e., is of lower degree than the volume 
function used for the averaging. In order to derive stability in $H_{h}^{\theta}$-norms, we would need a result analogous to Lemma 5.6. (1i) for the space $\left\{u \in \mathcal{P}_{p}(\widehat{T}),\left.\left.u\right|_{e} \in \mathcal{P}_{q} \wedge u\right|_{\partial \widehat{T} \backslash \widehat{e}}=0\right\}$, which is not available in the literature.

The proof of Lemma 6.3. (iii) can be adapted to the present case in a straight forward way.

\subsection{Proof of Theorems 2.5 and 2.6}

We only show the case for general meshes with all contributions in $\widetilde{\mathcal{S}}^{\mathbf{p}, 1}(\mathcal{T})$, the other cases follow analogously.

Proof of Theorem [2.6. Let $I_{h}^{1}$ denote an operator of Scott-Zhang type and $u_{1}:=u-I_{h}^{1} u$. Then

$$
\left\|u_{1}\right\|_{\widetilde{H}^{\theta}(\Gamma)}+\left\|h^{-\theta} u_{1}\right\|_{L^{2}(\Gamma)} \lesssim\|u\|_{\widetilde{H}^{\theta}(\Gamma)}
$$

For every vertex $V \in \mathcal{V}^{\text {int }}$ define $u_{V}:=\mathcal{A}_{\omega_{V}}^{p} u_{1}$ (see Corollary 6.2). By Lemma 6.1, (1i)

$$
\sum_{V \in \mathcal{V}^{i n t}}\left\|u_{V}\right\|_{\widetilde{H}_{h}^{\theta}\left(\omega_{V}\right)}^{2} \lesssim \sum_{V \in \mathcal{V}^{i n t}}\left\|u_{1}\right\|_{H_{h}^{\theta}\left(\omega_{V}\right)}^{2} \lesssim\|u\|_{\widetilde{H}^{\theta}(\Gamma)}^{2},
$$

where in the last step we used that the patches $\left(\omega_{V}\right)_{V \in \mathcal{V}^{i n t}}$ have finite overlap and (6.8). Extend $u_{V}$ by zero outside of $\omega_{V}$ and define $u_{\mathcal{V}}:=\sum_{V \in \mathcal{V}^{\text {int }}} u_{V}$. Set $u_{2}:=u_{1}-u_{\mathcal{V}}$. Let $d_{\mathcal{V}}:=\operatorname{dist}(\cdot, \mathcal{V})$ denote the distance to all vertices of $\mathcal{T}$. We claim the following estimates:

$$
\begin{aligned}
\left\|u_{2}\right\|_{\widetilde{H}^{\theta}(\Gamma)}+\left\|h^{-\theta} u_{2}\right\|_{L^{2}(\Gamma)} & \lesssim\|u\|_{\widetilde{H}^{\theta}(\Gamma)} \\
\left\|d_{\mathcal{V}}^{-\theta} u_{2}\right\|_{L^{2}(\Gamma)} & \lesssim\|u\|_{\widetilde{H}^{\theta}(\Gamma)} .
\end{aligned}
$$

To show (6.10), we note that since the contributions $u_{V}$ have local support, we can apply a coloring argument to localize the norms and apply the estimates (6.8) and (6.9)

$$
\left\|u_{2}\right\|_{\widetilde{H}^{\theta}(\Gamma)}^{2}+\left\|h^{-\theta} u_{2}\right\|_{L^{2}(\Gamma)}^{2} \lesssim\left\|u_{1}\right\|_{\widetilde{H}^{\theta}(\Gamma)}^{2}+\left\|h^{-\theta} u_{1}\right\|_{L^{2}(\Gamma)}^{2}+\sum_{V \in \mathcal{V}^{i n t}}\left\|u_{V}\right\|_{\widetilde{H}_{h}^{\theta}\left(\omega_{V}\right)}^{2} \lesssim\|u\|_{\widetilde{H}^{\theta}(\Gamma)}^{2} .
$$

To show (6.11), we consider a single element $K$ with vertices $\mathcal{V}_{K}:=\left\{V_{1}, \ldots, V_{k}\right\}$. By shape-regularity, $d_{\mathcal{V}} \sim d_{\mathcal{V}_{K}}$ on $K$, and we also have $d_{\mathcal{V}_{K}}^{-\theta}=\max _{j=1, \ldots, k} d_{V_{j}}^{-\theta} \leq \sum_{j=1}^{k} d_{V_{j}}^{-\theta}$. Using Lemma 6.1, (1ii) and (6.8), we conclude

$$
\begin{aligned}
\left\|d_{\mathcal{V}}^{-\theta} u_{2}\right\|_{L^{2}(K)}^{2} & \lesssim \sum_{j=1}^{k}\left\|d_{V_{j}}^{-\theta}\left(u_{1}-\sum_{\ell=1}^{k} u_{V_{\ell}}\right)\right\|_{L^{2}(K)}^{2} \\
& \lesssim \sum_{j=1}^{k}\left(\left\|d_{V_{j}}^{-\theta}\left(u_{1}-u_{V_{j}}\right)\right\|_{L^{2}(K)}^{2}+\sum_{\ell \neq j}\left\|d_{V_{j}}^{-\theta} u_{V_{\ell}}\right\|_{L^{2}(K)}^{2}\right) \lesssim \sum_{j=1}^{k}\left\|u_{1}\right\|_{H_{h}^{\theta}\left(\omega_{V_{j}}\right.}^{2} .
\end{aligned}
$$

Summing this estimate over all $K \in \mathcal{T}$ and using the second estimate of (6.9) shows (6.11). Next, define $u_{e}:=\mathcal{A}_{\omega_{e}}^{p} u_{2}$ for all edges $e \in \mathcal{E}^{i n t}$, using $\mathcal{A}_{\omega_{e}}^{p}$ from Corollary 6.4 Lemma 6.3, (1) and (6.10), (6.11) then imply

$$
\sum_{e \in \mathcal{E}^{i n t}}\left\|u_{e}\right\|_{\widetilde{H}_{h}^{\theta}\left(\omega_{e}\right)}^{2} \lesssim\|u\|_{\widetilde{H}^{\theta}(\omega)}^{2}
$$

Set $u_{\mathcal{E}}:=\sum_{e \in \mathcal{E}^{i n t}} u_{e}$ and $u_{3}:=u_{2}-u_{\mathcal{E}}$. We claim the estimates

$$
\begin{aligned}
\left\|u_{3}\right\|_{\widetilde{H}^{\theta}(\Gamma)}^{2}+\left\|h^{-\theta} u_{3}\right\|_{L^{2}(\Gamma)}^{2} & \lesssim\|u\|_{\widetilde{H}^{\theta}(\Gamma)}^{2}, \\
\left\|d_{\mathcal{E}}^{-\theta} u_{3}\right\|_{L^{2}(\Gamma)}^{2} & \lesssim\|u\|_{\widetilde{H}^{\theta}}^{2}(\Gamma) .
\end{aligned}
$$


(6.13) follows from (6.12) via the usual coloring argument to localize the norm. The weighted estimate (6.14) follows analogously to the proof of (6.11), using Lemma 6.3, (iii). Furthermore, (6.14) implies that for the restrictions $u_{K}:=\left.u_{3}\right|_{K}$ we can estimate $\left\|u_{K}\right\|_{\widetilde{H}_{h}^{\theta}(K)}^{2} \leq\|u\|_{\widetilde{H}^{\theta}(\Gamma)}^{2}$.

Proof of Theorem 2.5. Follows along the same lines as Theorem 2.6. one only has to replace the operators $\mathcal{A}_{\omega_{V}}^{p}$ and $\mathcal{A}_{\omega_{e}}^{p}$ with their continuous counterparts from Lemmas 6.1 and 6.3 respectively.

Acknowledgments: Financial support of A.R. by the Austrian Science Fund (FWF) through the doctoral school W1245 "Dissipation and Dispersion in Nonlinear PDEs" and the SFB 65 "Taming complexity in partial differential systems" is gratefully acknowledged. The research of the author M.K. is supported by Conicyt Chile through project FONDECYT 1170672 "Fast space-time discretizations for fractional evolution equations".

\section{REFERENCES}

[AF03] Robert A. Adams and John J. F. Fournier, Sobolev spaces, second ed., Pure and Applied Mathematics (Amsterdam), vol. 140, Elsevier/Academic Press, Amsterdam, 2003. MR 2424078

[AM03] Mark Ainsworth and William McLean, Multilevel diagonal scaling preconditioners for boundary element equations on locally refined meshes, Numer. Math. 93 (2003), no. 3, 387-413. MR 1953746 (2004i:65130)

[BB94] F. Ben Belgacem, Polynomial extensions of compatible polynomial traces in three dimensions, Comput. Meths. Appl. Mech. Eng. 116 (1994), 235-241.

[BDM92] C. Bernardi, M. Dauge, and Y. Maday, Trace liftings which preserve polynomials, C.R. Acad. Sci. Paris, Série I 315 (1992), 333-338.

[BDM07] - Polynomials in the Sobolev world (version 2), Tech. Report 14, IRMAR, 2007.

[BDM10] Christine Bernardi, Monique Dauge, and Yvon Maday, The lifting of polynomial traces revisited, Math. Comp. 79 (2010), no. 269, 47-69. MR 2552217 (2011b:46045)

[BM97] C. Bernardi and Y. Maday, Spectral methods, Handbook of Numerical Analysis, Vol. 5 (P.G. Ciarlet and J.L. Lions, eds.), North Holland, Amsterdam, 1997.

[Fed69] Herbert Federer, Geometric measure theory, Die Grundlehren der mathematischen Wissenschaften, Band 153, Springer-Verlag New York Inc., New York, 1969. MR 0257325

[FMPR15] T. Führer, J. M. Melenk, D. Praetorius, and A. Rieder, Optimal additive Schwarz methods for the hp-BEM: The hypersingular integral operator in 3D on locally refined meshes, Comput. Math. Appl. 70 (2015), no. 7, 1583-1605. MR 3396962

[FW15] Richard S. Falk and Ragnar Winther, The bubble transform: A new tool for analysis of finite element methods, Found. Comput. Math. (2015), 1-32 (English).

[Geo08] Emmanuil H. Georgoulis, Inverse-type estimates on hp-finite element spaces and applications, Math. Comp. $\mathbf{7 7}$ (2008), no. 261, 201-219 (electronic). MR 2353949 (2008j:65078)

[Heu99] Norbert Heuer, Additive Schwarz methods for indefinite hypersingular integral equations in $\mathbf{R}^{3}$-the p-version, Appl. Anal. 72 (1999), no. 3-4, 411-437. MR 1709072 (2000e:65124)

[Heu14] - On the equivalence of fractional-order Sobolev semi-norms, J. Math. Anal. Appl. 417 (2014), no. 2, $505-518$. MR 3194499

[HW08] George C. Hsiao and Wolfgang L. Wendland, Boundary integral equations, Applied Mathematical Sciences, vol. 164, Springer-Verlag, Berlin, 2008. MR 2441884 (2009i:45001)

[KMR] Michael Karkulik, Jens Markus Melenk, and Alexander Rieder, On interpolation spaces of piecewise polynomials on mixed meshes, in preparation.

[Lio88] Pierre-Louis Lions, On the Schwarz alternating method. I, First International Symposium on Domain Decomposition Methods for Partial Differential Equations (Paris, 1987), SIAM, Philadelphia, PA, 1988, pp. 1-42.

[Mad89] Y. Maday, Relèvement de traces polyômiales et interpolations hilbertiennes entres espaces de polynômes, C.R. Acad. Sci. Paris, Série I 309 (1989), 463-468.

[McL00] William McLean, Strongly elliptic systems and boundary integral equations, Cambridge University Press, Cambridge, 2000. MR 1742312 (2001a:35051)

[MN85] Aleksandr M. Matsokin and Sergey V. Nepomnyaschikh, A Schwarz alternating method in a subspace, Soviet Math. 29(10) (1985), 78-84.

[MW12] J.M. Melenk and B. Wohlmuth, Quasi-optimal approximation of surface based Lagrange multipliers in finite element methods, SIAM J. Numer. Anal. 50 (2012), 2064-2087.

[Pav94] L. Pavarino, Additive Schwarz methods for the p-version finite element method, Numer. Math. 66 (1994), 493-515.

[Sch98] Christoph Schwab, $p$ - and hp-finite element methods, Numerical Mathematics and Scientific Computation, The Clarendon Press, Oxford University Press, New York, 1998, Theory and applications in solid and fluid mechanics.

[SMPZ08] Joachim Schöberl, Jens M. Melenk, Clemens Pechstein, and Sabine Zaglmayr, Additive Schwarz preconditioning for p-version triangular and tetrahedral finite elements, IMA J. Numer. Anal. 28 (2008), no. 1, 1-24. MR 2387903 (2009a:74121) 
[SS11] Stefan A. Sauter and Christoph Schwab, Boundary element methods, Springer Series in Computational Mathematics, vol. 39, Springer-Verlag, Berlin, 2011, Translated and expanded from the 2004 German original. MR 2743235 (2011i:65003)

[Ste70] E.M. Stein, Singular integrals and differentiability properties of functions, Princeton University Press, 1970.

[Ste08] Olaf Steinbach, Numerical approximation methods for elliptic boundary value problems, Springer, New York, 2008, Finite and boundary elements, Translated from the 2003 German original. MR 2361676 (2008i:65271)

[Tar07] Luc Tartar, An introduction to Sobolev spaces and interpolation spaces, Lecture Notes of the Unione Matematica Italiana, vol. 3, Springer, Berlin, 2007. MR MR2328004 (2008g:46055)

[TS96] Thanh Tran and Ernst P. Stephan, Additive Schwarz methods for the h-version boundary element method, Appl. Anal. 60 (1996), no. 1-2, 63-84.

[TW05] Andrea Toselli and Olof Widlund, Domain decomposition methods-algorithms and theory, Springer Series in Computational Mathematics, vol. 34, Springer-Verlag, Berlin, 2005.

[vP89] Tobias von Petersdorff, Randwertprobleme der Elastizitätstheorie für Polyeder - Singularitäten und Approximation mit Randelementmethoden, Ph.D. thesis, Technische Hochschule Darmstadt, 1989

[Zha92] Xuejun Zhang, Multilevel Schwarz methods, Numer. Math. 63 (1992), no. 4, 521-539. 